Utah State University

DigitalCommons@USU

\title{
$5-2018$
}

\section{The Use of Microfluidics and Dielectrophoresis for Separation, Concentration, and Identification of Bacteria}

Cynthia Hanson

Utah State University

Follow this and additional works at: https://digitalcommons.usu.edu/etd

Part of the Biological Engineering Commons

\section{Recommended Citation}

Hanson, Cynthia, "The Use of Microfluidics and Dielectrophoresis for Separation, Concentration, and Identification of Bacteria" (2018). All Graduate Theses and Dissertations. 7044.

https://digitalcommons.usu.edu/etd/7044

This Dissertation is brought to you for free and open access by the Graduate Studies at DigitalCommons@USU. It has been accepted for inclusion in All Graduate Theses and Dissertations by an authorized administrator of DigitalCommons@USU. For more information, please contact digitalcommons@usu.edu.

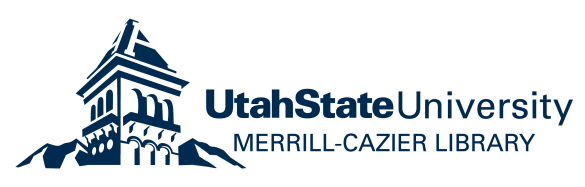


THE USE OF MICROFLUIDICS AND DIELECTROPHORESIS FOR SEPARATION, CONCENTRATION, AND IDENTIFICATION OF BACTERIA

by

Cynthia Hanson

A dissertation submitted in partial fulfillment

of the requirements for the degree

of

DOCTOR OF PHILOSOPHY

in

Biological Engineering

Approved:

Elizabeth A. Vargis, Ph.D.

Major Professor

Charles D. Miller, Ph.D.

Committee Member

Yu Huang, Ph.D.

Committee Member
Anhong Zhou, Ph.D.

Committee Member

Nicholas E. Dickenson, Ph.D.

Committee Member

Mark R. McLellan, Ph.D.

Vice President for Research and

Dean of the School of Graduate Studies

UTAH STATE UNIVERSITY

Logan, UT 
Copyright (C) Cynthia Hanson 2018

All Rights Reserved 


\begin{abstract}
The Use of Microfluidics and Dielectrophoresis for Separation, Concentration, and Identification of Bacteria

by

Cynthia Hanson, Doctor of Philosophy

Utah State University, 2018
\end{abstract}

Major Professor: Dr. Elizabeth Vargis

Department: Biological Engineering

Traditional bacterial analyses take one to two days under favorable conditions where the bulk of the time is spent waiting for bacteria to divide and grow until visual colonies can be observed for identification. In the case of bacteria with slow doubling times, this process can take weeks. This delay in analysis is unacceptable, especially in cases of life threatening diseases or emergencies. It is clear that in order to decrease the analysis time of the bacteria, the culturing and growth step must be circumvented. The goal of this research is to design, build, and test a device that could decrease the analysis time of bacteria using label-free methods of dielectrophoresis and Raman spectroscopy.

Testing for device design was performed with clinical samples in mind, which consist of bacteria grown in a variety of environmental conditions (i.e. available food sources, growth stage, temperature, etc.) and accompanied by sample debris. Raman spectra of bacteria grown in varying media and metabolic stages were collected and analyzed. Results indicate that growth phase and media have an impact on Raman spectra 
and is distinguishable by linear discriminant analysis (LDA). Despite these spectral differences, it was found that LDA classification of closely related bacteria remains fairly high (90\%) regardless of growth phase. Sample debris were also considered in device design and accommodated for by dielectrophoresis. Devices were built with the goal to isolate bacteria from a mixed sample and simultaneously acquire Raman spectra for identification.

For this dissertation, a device was designed, built, and tested that incorporates dielectrophoresis for particle isolation and Raman spectroscopy for identification. The device was modeled in COMSOL to ensure that an appropriate electrical field gradient could be obtained to isolate bacteria from $5 \mu$ m diameter polystyrene spheres. The device was built and successfully trapped bacteria away from polystyrene spheres and Raman spectra of the bacteria were collected while trapped. These results indicate a clear potential for contactless dielectrophoresis-Raman devices to isolate and identify bacteria from sample debris, and thereby decrease the analysis time of bacteria. 


\section{PUBLIC ABSTRACT}

The Use of Microfluidics and Dielectrophoresis for Separation,

Concentration and Identification of Bacteria

Cynthia Hanson

Typical bacterial analysis involves culturing and visualizing colonies on an array of agar plates. The growth patterns and colors among the array are used to identify the bacteria. For fast growing bacteria such as Escherichia coli, analysis will take one to two days. However, slow growing bacteria such as mycobacteria can take weeks to identify. In addition, there are some species of bacteria that are viable but nonculturable. This lengthy analysis time is unacceptable for life-threatening infections and emergency situations. It is clear that to decrease the analysis of the bacteria, the culturing and growth steps must be avoided. The goal of this research is to design, build, and test a device that could decrease the analysis time of bacteria.

Device design accommodates for the varied growth and environmental conditions of expected samples for bacterial analysis. Clinical samples containing bacteria come in a wide variety of forms including urine, saliva, sputum, blood, etc. Each medium will have associated debris and other contaminants that must be isolated from bacteria before identification. This process can be challenging as bacteria and debris can range in size from a fraction of a micrometer to tens of micrometers. In addition, a device must be equipped to accurately identify bacteria regardless of growth conditions. Thus, to decrease the analysis time of bacteria, a device must be capable of isolation, concentration, and identification at a micron level. 
In this dissertation, a device was designed, built, and tested that incorporates dielectrophoresis for cell sorting and Raman spectroscopy for identification. Using the device, bacteria (1 $\mu \mathrm{m}$ in length) were successfully isolated away from $5 \mu \mathrm{m}$ polystyrene spheres and Raman spectra of the trapped bacteria were collected. The simultaneous isolation and identification of bacteria from a mixed sample indicates the capability for the cDEP-Raman device to decrease the analysis time of bacteria from clinical samples. 


\section{ACKNOWLEDGMENTS}

Thank you to my family and my friends for their encouragement, love, and support as I've pursued my education. They have been instrumental in keeping my spirits up and making sure I took care of myself. Thank you to the BE staff for making my job easier as they have given of their time, advice, and expertise, especially Ann Martin, Jed Moss, Paul Veridian, Levi Sanchez, and Kami McNeil. I would especially like to thank Dr. Elizabeth Vargis for taking me on as a graduate student and providing me and others the opportunity to perform research here. Thank you to the other members of my research group for their suggestions, feedback, and advice for my research: Charles Harding, Farhad Farjood, and Nathan Israelsen. Thanks to the undergraduate students for their assistance in the lab: Karen Tew, Annelise Dykes, Michaela Salisbury, Michael Sieverts, Cameron Zabriskie, Jessica Burt, Ivy Hansen, Tessa Burrows, and Caleb Thomson. I would also like to recognize my committee members, Drs. Nicholas Dickenson, Yu Haung, Charles Miller, and Anhong Zhou for their time and advice.

This work was supported by the following awards, grants, and scholarships: Nuclear Regulatory Commission Faculty Development Award, USU Research Catalyst Award, USU Graduate Student Enhancement Award, USU Student Association, USU Dissertation Fellowship, USU Graduate Research and Creative Opportunities Award, USTAR Student Originated Project Award, USU College of Engineering Travel Grants, SPIE Optics and Photonics Scholarship, and the RGS Graduate Student Travel Award.

Lastly and most importantly, I thank God for placing these opportunities and great people along my path.

Cynthia Hanson 


\section{CONTENTS}

Page

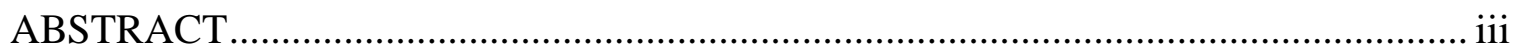

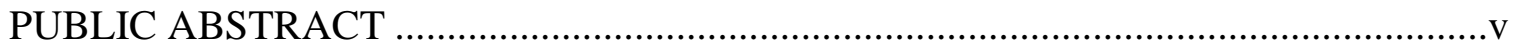

ACKNOWLEDGMENTS ................................................................................... vii

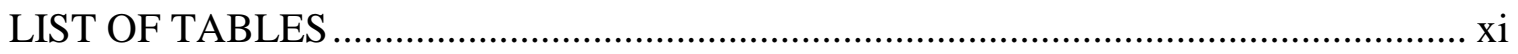

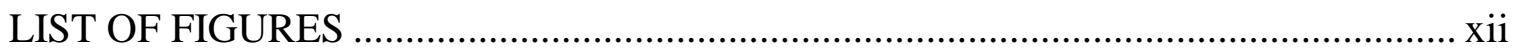

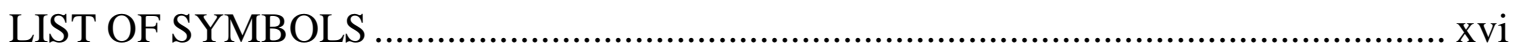

\section{CHAPTER}

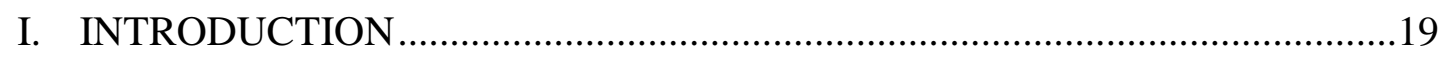

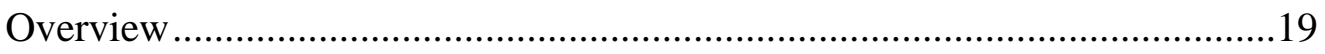

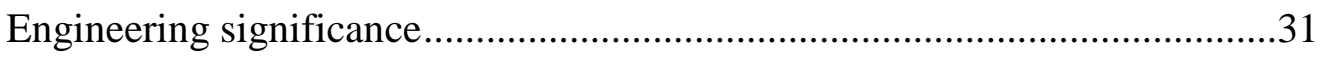

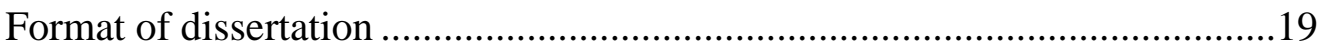

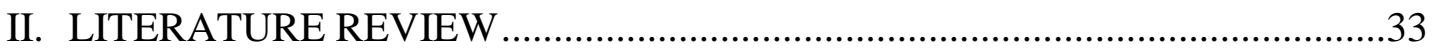

Raman spectroscopy for bacterial analysis ................................................33

DEP and Raman Spectroscopy for Bacterial Analysis ................................39

Motivation and Goals..........................................................................4 42

III. EFFECT OF PRINCIPAL COMPONENT ANALYSIS CENTERING AND SCALING ON CLASSIFICATION OF MYCOBACTERIA FROM RAMAN

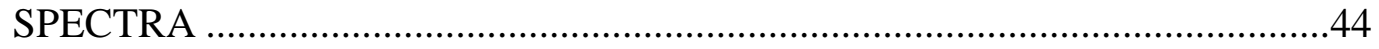

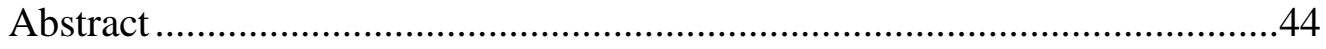

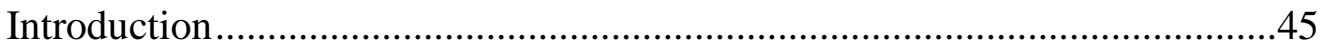

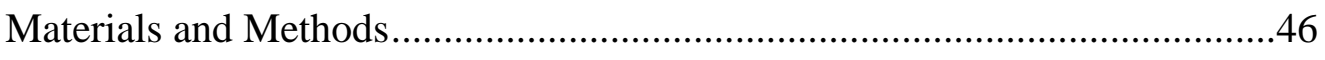

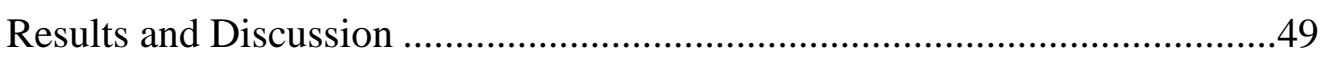

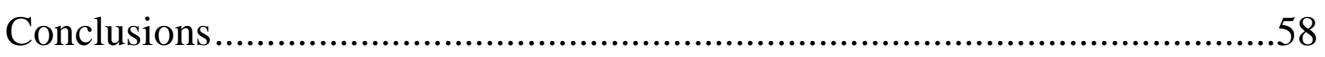


IV. INFLUENCE OF GROWTH MEDIA AND PHASE ON RAMAN

SPECTRA OF MYCOBACTERIA _.............................................................60

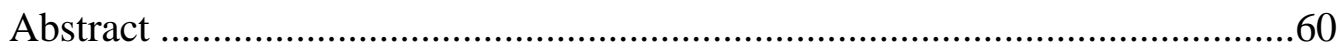

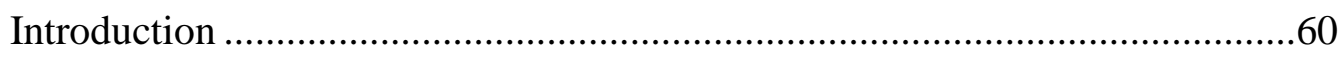

Materials and Methods .........................................................................64

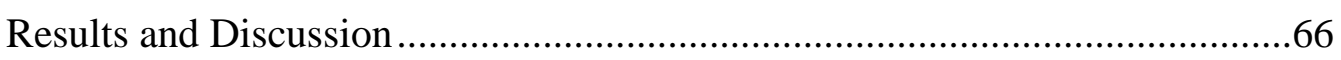

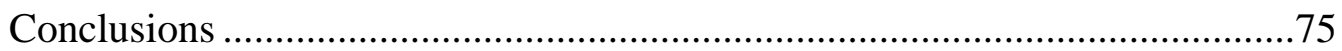

V. ALTERNATIVE CDEP DESIGN TO FACILITATE CELL ISOLATION FOR IDENTIFICATION BY RAMAN SPECTROSCOPY .............................77

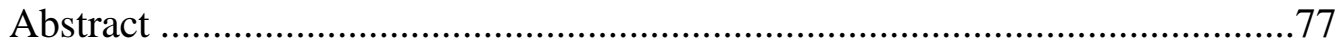

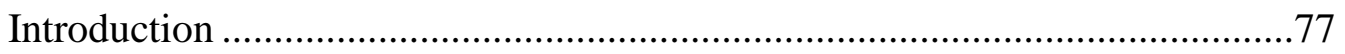

Materials and Methods ..........................................................................81

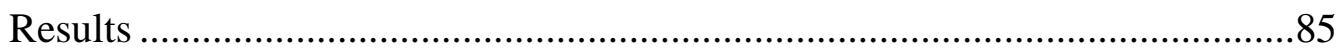

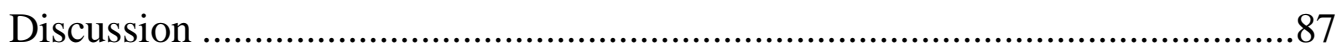

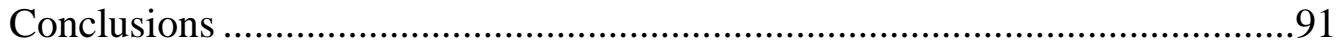

VI. SEPARATION OF A MIXED SAMPLE USING CDEP ................................92

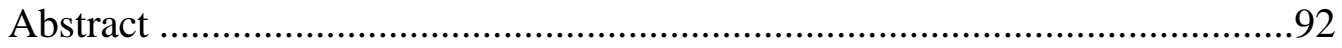

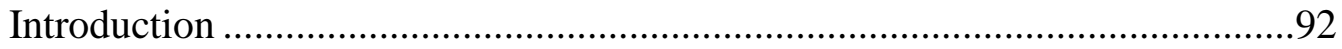

Materials and Methods .............................................................................94

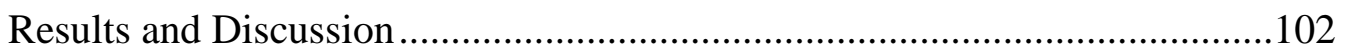

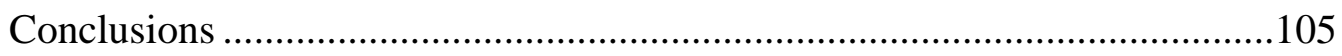

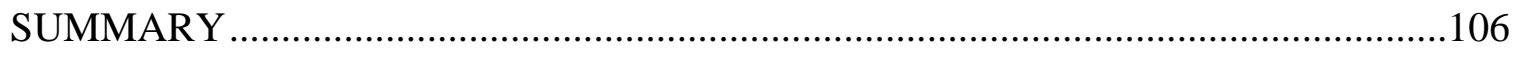

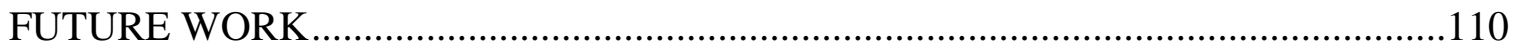

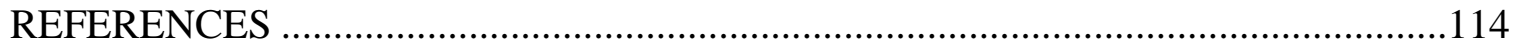

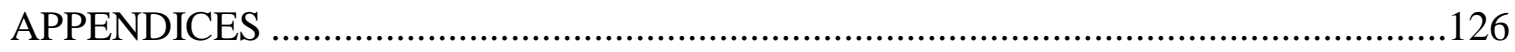

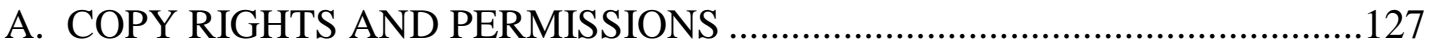


B. PERMISSION FROM AUTHORS TO REPRINT PUBLISHED

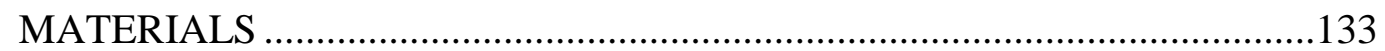

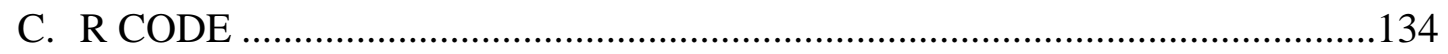

D. COMSOL OPERATING PARAMETERS ……………………………............144

E. LASTER ALIGNMENT ONTO SPECTROMETER SLIT ................................149

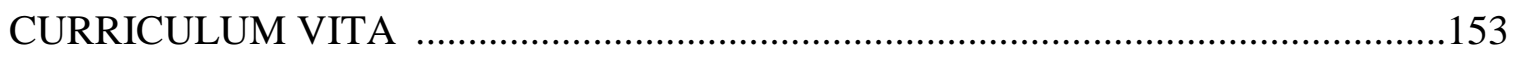




\section{LIST OF TABLES}

Table

Page

3.1. Assignment of spectra from each mycobacteria (Mycobacterium sp. JLS, Mycobacterium sp. KMS, and Mycobacterium sp. MCS) to form the test and training data sets.

4.1. Ingredient list for Lysogeny Broth, Brain Heart Infusion, Middlebrook, and Kirchner media. Contents are mixed with distilled water to make 1 liter of media.

4.2. Confusion matrix for LDA classification of Mycobacterium sp. MCS according to growth phase (log and stationary) and cultured in LB, BHI, Middlebrook, and Kirchner media. Classification results in 94.8\% accuracy.

4.3. Confusion matrices for LDA classification Mycobacterium sp. MCS grown in Lysogeny Broth (LB), Brain Heart Infusion (BHI), Kirchner (K), and Middlebrook (M) media. Matrices are grouped according to log and stationary phases, which have classification accuracies of $85.4 \%$ and $93.8 \%$, respectively

5.1. List of cDEP publications and their associated operating parameters. As the list consists of alternating current sources, voltage is expressed in root mean square ( $\mathrm{V}_{\mathrm{RMS}}$ ).

6.1. List of electrical permittivities, electrical conductivities, frequency, and the Clausius-Mossotti factor used to determine the required electric field gradient to induce trapping of bacteria and polystyrene spheres.

D.1. Table of parameters under Global Definitions 


\section{LIST OF FIGURES}

Figure

Page

1.1. Energy diagram depicting Rayleigh and Raman scattering with dashed lines indicating virtual energy states. $S_{0}$ and $S_{1}$ represent the ground and an excited electronic state of the molecule respectively. Thin black horizontal lines represent the vibrational states within their respective electronic state. Arrows pointing up represent excitation while arrows pointing down represent scattering.

1.2. Illustration of various vibrational modes for carbon dioxide and associated plots of polarizability $(\alpha)$ as a function of the normal coordinates of each vibrational mode $(Q)$.

1.3. Raman spectra of Mycobacterium sp. JLS, Mycobacterium sp. KMS, and Mycobacterium sp. MCS.

1.4. Illustration of electrophoresis (a) and dielectrophoresis (b). Thin black lines indicate electric field lines while the vertical black lines indicate electrodes. Blue and green arrows indicate forces acting on particles.

1.5. Illustration of the shape, axes, and layers associated with Mycobacterium with $a$ and $b$ indicating major and minor axes of the bacteria respectively. Pairing layers from the core to the outer most layer (b, c, and d) is done to determine intermediate and overall effective electrical permittivities ( $\left.\varepsilon_{\text {eff }}\right)$.

3.1. Examples of the original spectra (a), baseline corrected spectra (b), normalized spectra (c), and spectra from each mycobacteria collected with a 10s acquisition time and six accumulations (d). Spectra were taken of Mycobacterium sp. JLS, Mycobacterium sp. KMS, and Mycobacterium sp. MCS.

3.2. The highest classification accuracies possible for data which were centered and scaled, uncentered and scaled, centered and unscaled, and uncentered and unscaled during PCA.

3.3. Examples of scree plots from data set 3 which were scaled and centered, scaled and uncentered, unscaled and centered, and unscaled and uncentered during PCA. Note the varying scales of variances in the $y$-axis for each condition.

3.4. Average drop in classification accuracy from the maximum accuracy possible for choosing PCs based on the scree plot method, Kaiser method, and cumulative percent variance (CPV) covered by the PCs. The 
secondary axis in red indicate the best classification accuracies (averaged over six data sets) with error bars indicating standard deviation.

3.5. Linear discriminant analysis classification accuracy as a function of PCs for all centering and scaling options from data set 3. 56

3.6. Plot of PCs 1, 3, and 5 from data set 3 which was centered and unscaled. Blue, green, and red dots represent Mycobacterium sp. JLS, Mycobacterium sp. MCS, and Mycobacterium sp. KMS, respectively. 58

4.1. Growth curve for Mycobacterium sp. MCS in LB media based on optical density measurements at $580 \mathrm{~nm}$. Error bars at each point represent the standard deviation among seven technical replicates.

4.2. Linear discriminant analysis plot of spectra from Mycobacterium sp. MCS at the stationary (index 1-48) and log (index 49-96) phases. Blue and green text indicates the predicted stationary and log phases, respectively. Classification results in $94.8 \%$ accuracy.

4.3. Plot of linear discriminants of Raman spectra of Mycobacterium sp. MCS at the log phase according to culture media. Media included Brain Heart Infusion (BHI), Lysogeny broth (LB), Middlebrook (M), and Kirchner (K). The plot displays the actual media by color and the predicted media by shape as indicated by the legend. Classification resulted in $85.4 \%$ accuracy.

4.4. Plot of linear discriminants of Raman spectra of Mycobacterium sp. MCS at the stationary phase according to culture media. Media included Brain Heart Infusion (BHI), Lysogeny broth (LB), Middlebrook (M), and Kirchner (K). The plot indicates the actual media by color and the predicted media by shape as indicated by the legend. Classification resulted in $93.8 \%$ accuracy.

4.5. Raman spectra of Mycobacterium sp. MCS grown in Lysogeny Broth (LB), Middlebrook (M), Kircher (K), and Brain Heart Infusion (BHI) media for the log and stationary phases represented by blue and orange lines, respectively.

4.6. Raman spectra of Mycobacterium sp. MCS from $750-1600 \mathrm{~cm}^{-1}$ for Lysogeny broth LB, Middlebrook (M), Kircher (K), and Brain Heart Infusion (BHI) media for the log (blue) and stationary (orange) phases. Peaks highlighted in red, gray, and green mark peaks associated with DNA, carotenoids, and mycolic acids, respectively.

5.1. Illustration of the layered microfluidic device. Top and bottom plates were $3 \mathrm{D}$ printed with holes to accommodate \#8-32 screws. The bottom plate is equipped with a viewing port for an inverted microscope. The second 
layer from the top is made of PDMS. The second plate from the bottom is the fused silica microfluidic plate.

5.2. (a) Illustration of the fused silica microfluidic chip. Liquid electrodes and the sample channels are indicated by blue and red lines, respectively. The array of 4 by 15 square pillars act as insulating barriers in the middle of the sample channel. (b) Microscope image of the middle of the microfluidic sample channel showing the array of pillars in the middle of the sample channel. Pillar dimensions are $100 \mu \mathrm{m}$ by $100 \mu \mathrm{m}$.

5.3. Image of the layered microfluidic device in operation set a-top an inverted microscope for analysis.

5.4. Image of cDEP device under operation ( $\left.350 \mathrm{~V}_{\mathrm{RMS}}, 100 \mathrm{~Hz}, 5 \mu \mathrm{L} / \mathrm{h}\right)$, trapping polystyrene spheres with a diameter of $3.3 \mu \mathrm{m}$. Square pillars are $100 \mu \mathrm{m}$ by $100 \mu \mathrm{m}$. 86

5.5. Raman spectra of $3.3 \mu \mathrm{m}$ PSS trapped within the cDEP device (black), PSS on quartz coverslip (blue), PDMS (green), and the quartz coverslip with $0.005 \times$ PBS (red). The spectrum of PSS on a quartz coverslip (blue) is the positive control. The spectra of PDMS (green) and the quartz coverslip without PSS (red) are negative controls.

6.1. COMSOL simulations to determine expected gradient of the squared electric field for first (a) and second (b) pillar array operated at $500 \mathrm{~V}$ and $40 \mathrm{kHz}$. Units for values are in $\mathrm{kg}^{2} \mathrm{~m} / \mathrm{s}^{6} \mathrm{~A}^{2}$

6.2. Schematic of layered cDEP device. 100

6.3. Schematic of fused silica microfluidic plate (a) with green and blue channels, indicating the liquid electrode and sample channels, respectively. Brightfield images of first (b) and second (c) pillar arrays positioned in the middle of the sample channel. In (b), pillars are $60 \mu \mathrm{m}$ diameter while in (c), the length of and width of the oval pillars are 100 and $60 \mu \mathrm{m}$, respectively. 100

6.4. Brightfield images of isolating bacteria away from polystyrene spheres acting as debris. Image (a) is of the first pillar array with pillar diameters for $60 \mu \mathrm{m}$, while image (b) is of the second pillar array with the length of the oval pillar being $100 \mu \mathrm{m}$. The red circle indicates the area where Raman spectra were collected.

6.5. A Raman spectrum of Mycobacterium sp. MCS isolated from $5 \mu \mathrm{m}$ polystyrene spheres while trapped in the cDEP-Raman device.

6.6. Raman spectra of Escherichia coli (black), Mycobacterium sp. MCS (blue), Pseudomonas putida (green), and Streptococcus bovis (red). 105 
E.1. Image of laser delivery to in-house built Raman microscope. Important components include laser head (A), filter wheel with neutral density filters (B), $60 \mathrm{~mm}$ to $30 \mathrm{~mm}$ adapter plates with threaded hole to accommodate alignment target (C1 and C2), mirrors in kinematic mounts (D1 and D2), entrance to spectrometer slit (E), and the spectrometer (F).

E.2. Image of light entering through the spectrometer slit to determine vertical and horizontal midpoint for laser alignment.

E.3. Image of laser spot being aligned to the center of the spectrometer slit as determined by Figure E.2.

E.4. Illustration of filter cube used in the in-house built Raman microscope. Blue and red lines represent the excitation laser and the scattered light, respectively. 


\section{LIST OF SYMBOLS}

Symbol

A

$a$

b

C

$\left[f_{C M}\right]$

E

e

$f$

h

j

Q

$r$

$R e$

$S$

$U$

\section{Greek Symbols}

$\alpha$
$\beta$
$\varepsilon_{o}$
$\varepsilon_{i}$
$\varepsilon^{*}$
$\gamma$
$\eta$
$\lambda$
$\pi$
$\sigma$

Description

Unit

Depolarization factor

Major axis of prolate ellipsoid

m

Minor axis of prolate ellipsoid

m

Speed of light

$\mathrm{m} / \mathrm{s}$

Clausius-Mossotti factor

Energy

J

Eccentricity

Frequency

$\mathrm{s}^{-1}$

Planck's constant

$\mathrm{m}^{2} \mathrm{~kg} / \mathrm{s}$

Square root of negative 1

Vibrational mode

Particle radius

m

Real part

Electronic state

Velocity

$\mathrm{m} / \mathrm{s}$

\section{Polarization}

See Equation (6.12)

Electrical permittivity of free space

$\mathrm{F} / \mathrm{m}$

Relative electrical permittivity

Complex electrical permittivity

$\mathrm{F} / \mathrm{m}$

$a / b$, ratio of the major and minor axes of a prolate ellipsoid

Kinematic viscosity

$\mathrm{m}^{2} / \mathrm{s}$

Wavelength

m

Pi

Electrical conductivity 


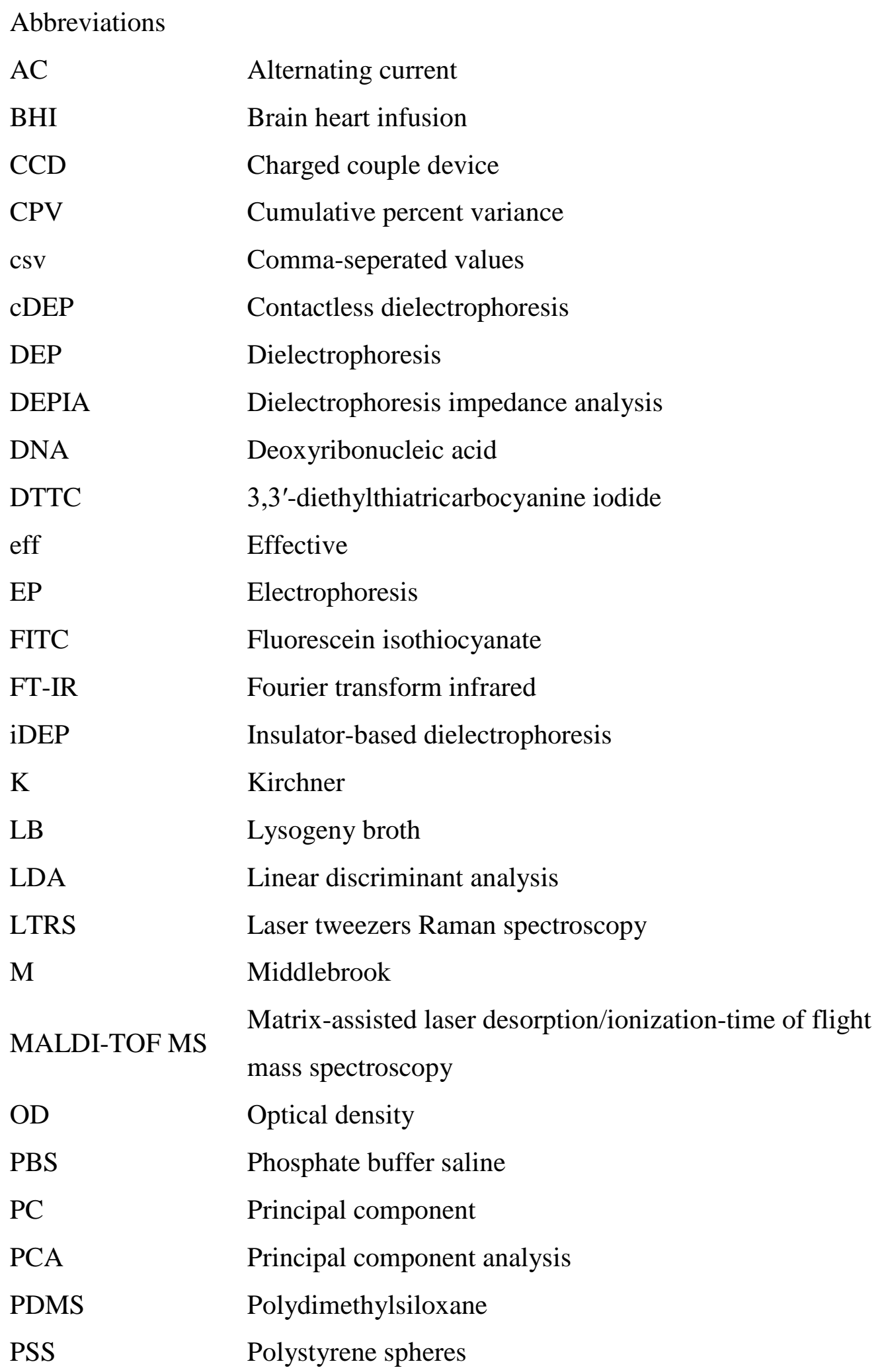




$\begin{array}{ll}\text { PTTE } & \text { Polytetrafluoroethylene } \\ r m s & \text { Root mean square } \\ \text { RNA } & \text { Ribonucleic acid } \\ \text { RR } & \text { Resonance Raman } \\ \text { TRITC } & \text { Tetramethylrhodamine isothiocyante } \\ \text { UV-Vis } & \text { Ultraviolet visible }\end{array}$




\section{CHAPTER I}

\section{INTRODUCTION}

\section{Format of dissertation}

Chapter 1 of this dissertation is an introduction to the research, covering concepts of Raman spectroscopy, dielectrophoresis, and the importance of developing a technique to promptly and accurately identify bacteria. Chapter 2 contains a literature review covering the use of Raman spectroscopy and dielectrophoresis to identify and isolate bacteria. Chapter 3 is an article published in the journal Applied Spectroscopy [1] addressing how scaling and centering of spectral data during Principal Component Analysis influences classification results of mycobacteria. Chapter 4 is a manuscript in preparation for publication in a peer reviewed journal. It covers the influence of growth media and phase on Raman spectra of Mycobacterium sp. MCS. Chapter 5 is an article published in the journal Sensors [2] demonstrating the use of an alternative cDEP design to simultaneously trap and analyze polystyrene spheres. Chapter 6 is a manuscript in preparation for publication in a peer reviewed journal. It covers the use of a similar cDEP device to simultaneously isolate, trap, and identify a mixed sample of bacteria and polystyrene spheres. Chapter 7 is a summary of the work, Chapter 8 is suggestions for future work, and Chapter 9 contains all references cited throughout this work. The appendices include copyright permission, the author's curriculum vitae, R-code, COMSOL operating parameters, and laser alignment protocol.

\section{Overview}

The goal of this research is to design, build, and test a device for simultaneous isolation and identification of cells in order to decrease the time required to identify 
bacteria in a sample. Current bacterial identification methods require visual confirmation of colonies grown on an array of agar plates, taking one to two days for fast growing bacteria. This lengthy analysis time is unacceptable especially for slow growing bacteria, viable but nonculturable bacteria, or life-threatening infections. In this dissertation, the analysis time will be decreased by simultaneously isolating cells using dielectrophoresis (DEP) and identifying them using Raman spectroscopy. This unique design provides a platform for prompt and accurate bacterial identification.

\subsection{Introduction to Raman spectroscopy}

Raman spectroscopy is a laser spectroscopy technique used to analyze shifts in rotational and vibrational energy levels of molecules. A comprehensive explanation of Raman spectroscopy can be found throughout literature [3-8]. A brief introduction incorporating the classical approach to explaining Raman scattering and the associated selection rules are given here.

When a molecule is exposed to light, the induced dipole moment can be express as

$$
\mathbf{p}=\alpha \cdot \mathbf{E}
$$

where $\alpha$ is the polarizability and $\mathbf{E}$ is the electric field caused by a laser (electromagnetic radiation). The electric field is expressed as

$$
\mathbf{E}=\mathbf{E}_{o} \cos \left(2 \pi \cdot v_{o} \cdot t\right)
$$

Here, the subscript, o, indicates the initial state while $v_{0}$ is the vibrational frequency of the electromagnetic radiation. The polarizability is influenced by how the molecule 
moves (vibrational and rotational states) and is dependent on $Q$, the normal coordinate of the molecule. Using the Taylor Series, the polarizability is expressed as

$$
\alpha=\alpha_{o}+\sum_{k}\left(\frac{\partial \alpha}{\partial Q_{k}}\right)_{o} \cdot Q_{k}+\frac{1}{2} \sum_{k, l}\left(\frac{\partial^{2} \alpha}{\partial Q_{k} \partial Q_{l}}\right)_{o} \cdot Q_{k} \cdot Q_{l}+\cdots
$$

with subscripts $k$ and $l$ corresponding to the $k^{\text {th }}$ and $l^{\text {th }}$ normal vibrations. Assuming the different normal vibrations are independent, Equation (1.3) can be simplified to the first approximation as follows

$$
\alpha_{v}=\alpha_{o}+\alpha_{v}^{\prime} \cdot Q_{v}
$$

with the subscript $v$ representing the $v^{\text {th }}$ normal vibration and $\alpha_{v}^{\prime}$ represents the derivative of the polarizability tensor under equilibrium conditions. In addition, $Q_{v}$ is expressed as

$$
Q_{v}=Q_{v o} \cdot \cos \left(2 \pi \cdot v_{v} \cdot t+\varphi_{v}\right)
$$

where $\varphi_{v}$ is the phase angle and $Q_{v o}$ is the amplitude of the normal vibration. Upon substitution of variables expressed in Equations (1.1), (1.2), (1.4), and (1.5), gives:

$$
\mathbf{p}=\alpha_{o} \mathbf{E}_{o} \cos \left(2 \pi \cdot v_{o} \cdot t\right)+\alpha_{v}^{\prime} \mathbf{E}_{o} Q_{v o} \cdot \cos \left(2 \pi \cdot v_{o} \cdot t\right) \cdot \cos \left(2 \pi \cdot v_{v} \cdot t+\varphi_{v}\right)
$$

Using the trigonometrical identity

$$
\cos A \cdot \cos B=\frac{1}{2}[\cos (A+B)+\cos (A-B)]
$$

Equation (1.6) can be rewritten as

$$
\begin{gathered}
\mathbf{p}=\alpha_{o} \mathbf{E}_{o} \cos \left(2 \pi \cdot v_{o} \cdot t\right)+\frac{1}{2} \alpha_{v}^{\prime} \mathbf{E}_{o} Q_{v o} \cdot \cos \left[2 \pi \cdot\left(v_{o}+v_{v}\right) \cdot t+\varphi_{v}\right]+\frac{1}{2} \alpha_{v}^{\prime} \mathbf{E}_{o} Q_{v o} \\
\cos \left(2 \pi \cdot\left(v_{o}-v_{v}\right) \cdot t-\varphi_{v}\right)
\end{gathered}
$$

Assuming the dipole moment is a function of the vibrational frequency of the incident radiation $\left(v_{o}\right)$ and the molecule $\left(v_{v}\right)$, the dipole moment can be described as

$$
\mathbf{p}=\mathbf{p}\left(v_{o}\right)+\mathbf{p}\left(v_{o}+v_{v}\right)+\mathbf{p}\left(v_{o}-v_{v}\right)
$$


The first term of Equation (1.9) indicates no molecular vibrational change. This means the frequency of the light scattering from a molecule is the same as the frequency of light incident of the molecule. This is called Rayleigh scattering. The second and third terms of Equation (1.9) indicate a change in frequency of the incident and scattered light and is referred to as Raman scattering. Increasing of frequency $\left(v_{o}+v_{v}\right)$ is referred to as AntiStokes scattering while decreasing of frequency $\left(v_{o}-v_{v}\right)$ is referred to as Stokes scattering. Figure 1.1 is provided to help illustrate Rayleigh and Raman scattering and how each form of scattering influences molecular vibrational energy.

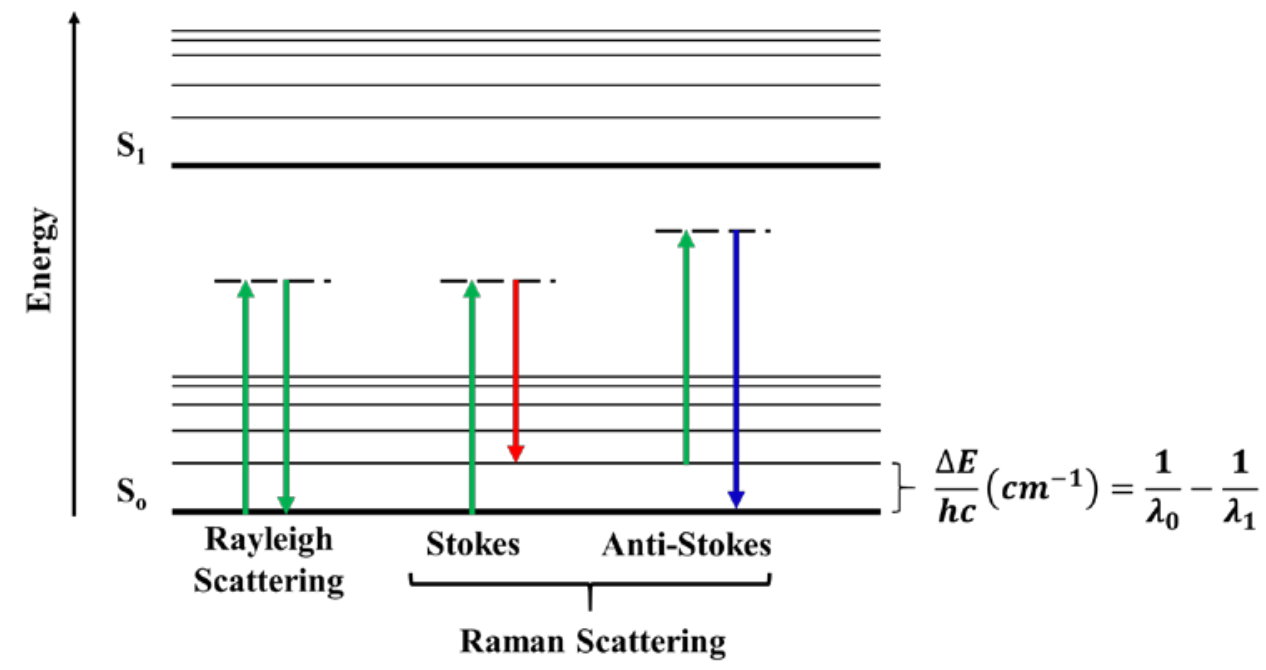

Figure 1.1. Energy diagram depicting Rayleigh and Raman scattering with dashed lines indicating virtual energy states. $\mathrm{S}_{\mathrm{o}}$ and $\mathrm{S}_{1}$ represent the ground and an excited electronic state of the molecule, respectively. Thin black horizontal lines represent the vibrational states within their respective electronic state. Arrows pointing up represent excitation while arrows pointing down represent scattering. 
The change in molecular energy during Raman scattering is often referred to as relative wavenumbers and describes the relationship between frequency and wavelength ( $\lambda$ ) when considering the equation for energy of a photon.

$$
E=h v=\frac{h c}{\lambda}
$$

Here, $h$ is Planck's constant and $c$ is the speed of light. Wavenumbers $\left(\mathrm{cm}^{-1}\right)$ are introduced as illustrated in Figure 1.1.

It should be noted that not all changes in molecular vibrations are Raman active. Raman scattering is dependent upon $\alpha_{v}^{\prime} \neq 0$. Figure 1.2 is provided to illustrate active and inactive vibrational modes using carbon dioxide as an example. Vibrational modes of symmetric stretching, asymmetric stretching, and bending are represented along with a plot of $\alpha$ as a function of $Q$. For a molecule to be Raman active, the derivative of the polarizability with respect to the normal coordinate of the molecule at equilibrium $\left(Q_{0}\right)$ cannot be equal to zero. In other words, $\left(\frac{\partial \alpha}{\partial Q_{v}}\right)_{o} \neq 0$. Therefore, asymmetric stretching and bending are Raman inactive vibrational modes, while symmetric stretching is Raman active for carbon dioxide. 


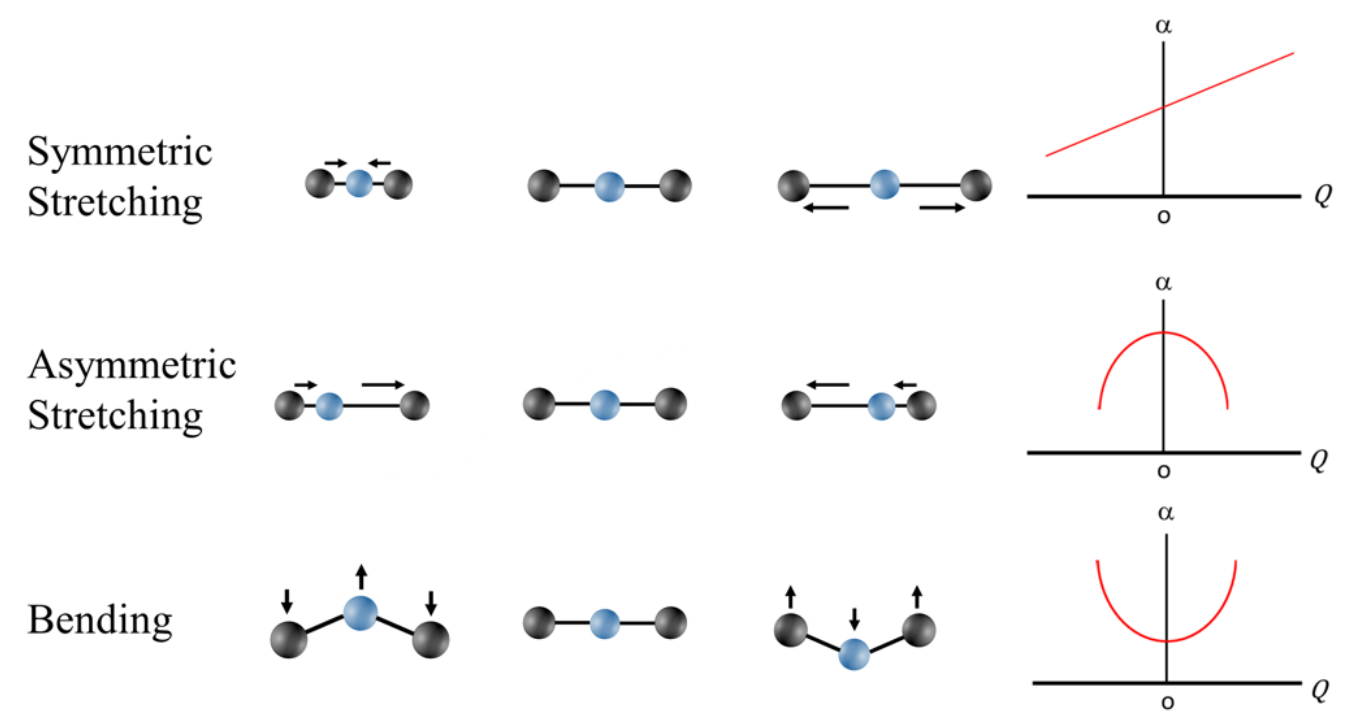

Figure 1.2. Illustration of various vibrational modes for carbon dioxide and associated plots of polarizability $(\alpha)$ as a function of the normal coordinates of each vibrational mode $(Q)$.

For complex structures, multiple Raman active vibrational modes are observed. The combination of wavenumbers and their associated intensities are compiled to form a spectrum that can be used as a means of identification and is often referred to as a Raman signature or fingerprint. Raman spectra of bacteria studied in this research (Mycobacterium sp. JLS, Mycobacterium sp. KMS, and Mycobacterium sp. MCS) are displayed in Figure 1.3. With the aid of multivariate statistics, minute changes between spectra can be detected and used to discriminate between bacteria types, species, and strains. 


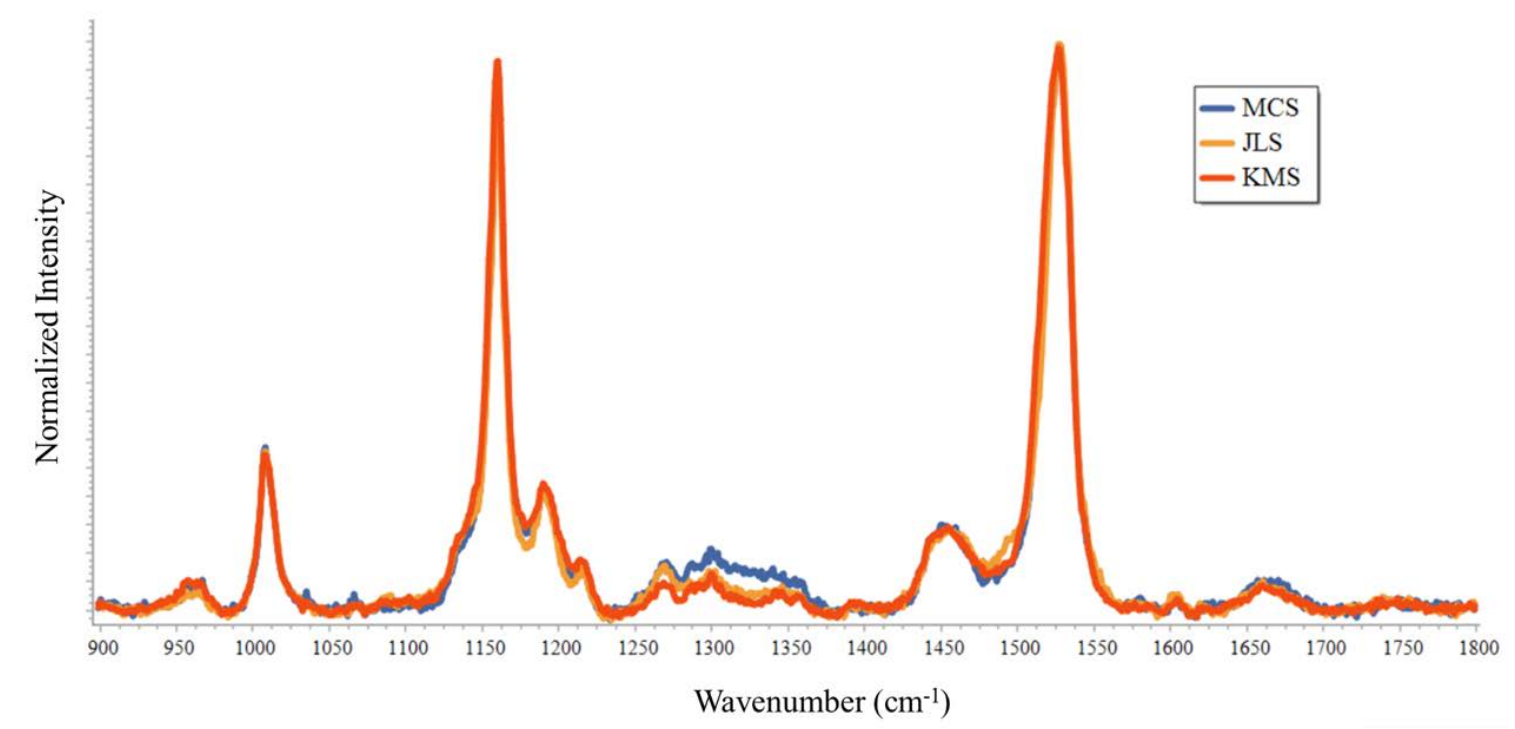

Figure 1.3. Raman spectra of Mycobacterium sp. JLS, Mycobacterium sp. KMS, and Mycobacterium sp. MCS.

\subsection{Introduction to dielectrophoresis}

Dielectrophoresis is the movement of particles in a non-uniform electric field. The movement is due to particles forming dipoles while in the presence of an electric field. Figure 1.4 illustrates the concept of electrophoresis and DEP. In electrophoresis (Figure 1.4a), only charged particles can migrate while neutral particles stay in place as all forces are balanced. In DEP (Figure 1.4b), charged and neutral particles will migrate as a net force is caused by the dipole formation and gradient of the electrical field. The force acting on the particle is a function of several properties including particle shape, size, homogeneity, and electrical properties. In addition, the electrical properties of the surrounding media and the gradient of the electrical field also contribute to the magnitude and direction of dielectric force. 
(a)

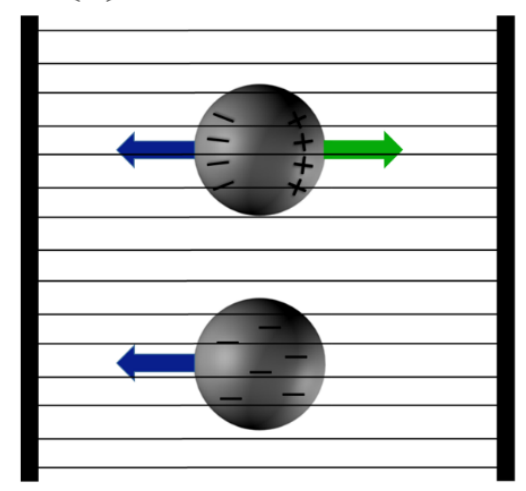

(b)

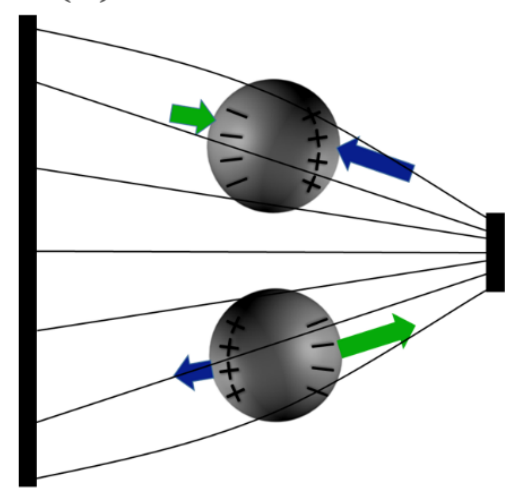

Figure 1.4. Illustration of electrophoresis (a) and dielectrophoresis (b). Thin black lines indicate electric field lines while the vertical black lines indicate electrodes. Blue and green arrows indicate forces acting on particles.

The dielectrophoretic force acting on a spherical, homogenous particle is expressed as

$$
\vec{F}_{D E P}=2 \pi r^{3} \varepsilon_{m} \operatorname{Re}\left[f_{C M}\right] \nabla\left(\vec{E}_{r m s}^{2}\right)
$$

where $\mathrm{r}, \nabla\left(\vec{E}_{r m s}^{2}\right)$, and $\varepsilon_{\mathrm{m}}$ are the radius of a sphere, gradient of the squared electric field, and the electrical permittivity of the media, respectively. The subscript $r m s$ stands for root mean square and is applicable for electric fields generated by AC power sources. $\operatorname{Re}\left[f_{C M}\right]$ is the real part of the Clausius-Mossotti factor, which is expressed as

$$
\left[f_{C M}\right]=\frac{\varepsilon_{p}^{*}-\varepsilon_{m}^{*}}{\varepsilon_{p}^{*}+2 \varepsilon_{m}^{*}}
$$

where $\varepsilon_{p}^{*}$ and $\varepsilon_{m}^{*}$ are the complex electrical permittivity of the particle and media respectively. The complex permittivity is dependent on the conductivity $(\sigma)$ and the frequency of the applied electric field $(f)$ and is given by

$$
\varepsilon^{*}=\varepsilon-\frac{j \sigma}{2 \pi f}
$$


where $j$ is the square root of negative one.

The dielectrophoretic force is dependent on factors such as shape and heterogeneity of the particle. As the majority of the work in this dissertation deals with mycobacteria, equations to determine DEP force on a multilayered, prolate ellipsoid will be given in the equations below. Assuming mycobacteria have the shape of a prolate ellipsoid, Equation (1.11) changes to

$$
\vec{F}_{D E P}=\frac{2 \pi a b^{2}}{3} \varepsilon_{m} R e\left[f_{C M}\right] \nabla\left(\vec{E}_{r m s}^{2}\right)
$$

where $a$ and $b$ are the major and minor axis of a prolate ellipsoid. The calculation of the Clausius-Mossotti factor is expressed as

$$
\left[f_{C M}\right]=\frac{\varepsilon_{p}^{*}-\varepsilon_{m}^{*}}{1+\left(\frac{\varepsilon_{p}^{*}-\varepsilon_{m}^{*}}{\varepsilon_{m}^{*}}\right) A}
$$

Here, $A$ is the depolarization factor and is dependent on the respective $\mathrm{x}, \mathrm{y}$, and $\mathrm{z}$ axes. However, in the case of a prolate ellipsoid the depolarization can be expressed by the expansion

$$
A=\frac{1}{3 \gamma^{-2}}\left[1+\frac{3}{5}\left(1-\gamma^{-2}\right)+\frac{3}{7}\left(1-\gamma^{-2}\right)^{2}+\cdots\right]
$$

where $\gamma$ is the major axis ( $a$ ) of the prolate ellipsoid divided by the minor axis $(b)$ as indicated in Figure 1.5a.

The expression for the Clausius-Mossotti factor is also influenced by heterogeneity. For example, bacteria often have several layers. In the case of Mycobacterium, a core-shell model can be used with the cytoplasm being considered the core $\left(a_{4}, b_{4}, \varepsilon_{5}, \sigma_{5}\right)$, the cytoplasmic membrane as the first shell $\left(a_{3}, b_{3}, \varepsilon_{4}, \sigma_{4}\right)$, the cell wall as the second shell $\left(a_{2}, b_{2}, \varepsilon_{3}, \sigma_{3}\right)$, and the lipid layer as the third shell $\left(a_{1}, b_{1}, \varepsilon_{2}, \sigma_{2}\right)$ as depicted in Figure 1.5a. For core-shell configurations, the overall effective electrical 
permittivity must be determined and used in place of $\varepsilon_{p}^{*}$ from Equation (1.13) to calculate $\left[f_{C M}\right]$. Using Figure 1.5b-d as a guide for notation, the effective electrical permittivity is expressed as

$$
\varepsilon_{(i) \mathrm{eff}}^{*}=\varepsilon_{i}^{*}\left[\frac{\varepsilon_{i}^{*}+\left(\varepsilon_{(i+1) \mathrm{eff}}^{*}-\varepsilon_{i}^{*}\right)\left(A_{i}+v_{i}\left(1-A_{(i-1)}\right)\right)}{\varepsilon_{i}^{*}+\left(\varepsilon_{(i+1) \mathrm{eff}}^{*}-\varepsilon_{i}^{*}\right)\left(A_{i}+v_{i} A_{(i-1)}\right)}\right]
$$

with

$$
v_{i}=\frac{a_{i} b_{i}^{2}}{a_{i-1}^{3}} \quad[9,10]
$$

(a)

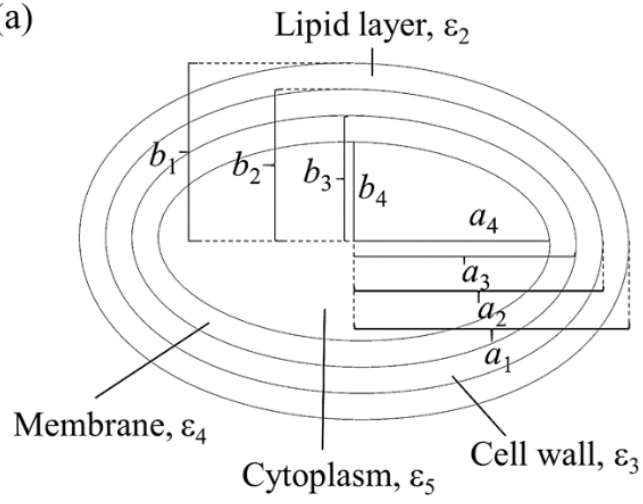

(c)

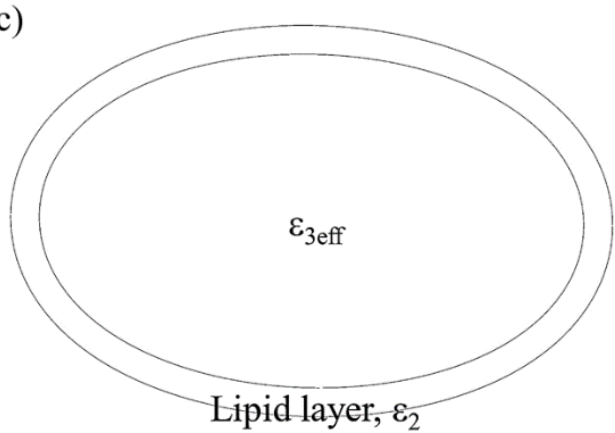

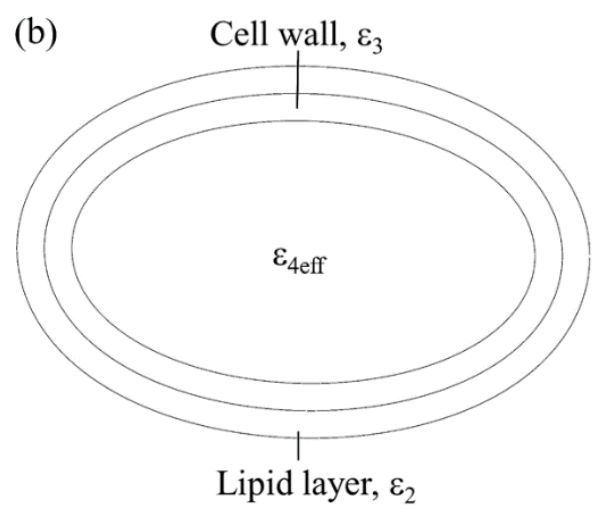

(d)

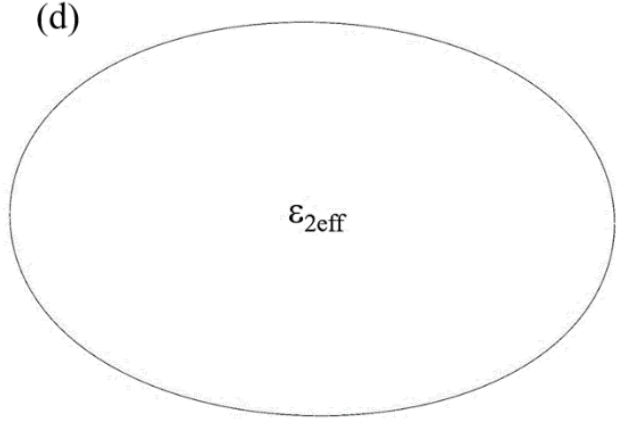

Figure 1.5. Illustration of the shape, axes, and layers associated with Mycobacterium with $a$ and $b$ indicating major and minor axes of the bacteria respectively. Pairing layers from the core to the outer most layer (b, c, and d) is done to determine intermediate and overall effective electrical permittivities ( $\left.\varepsilon_{\mathrm{eff}}\right)$. 
Calculation of $\left[f_{C M}\right]$ is important in determining the direction a particle will migrate due to the dielectrophoretic force acting upon it. When it is positive, the dielectrophoretic force on the particle will push the particle to a high electric field gradient. In contrast, when $\left[f_{C M}\right]$ is negative, the dielectrophoretic force will push the particle away from a high electric field gradient. Therefore, the direction of the dielectrophoretic force is based on the electric field gradient and the $\left[f_{C M}\right]$ rather than positioning of anode or the cathode. Using these concepts, a particle can be isolated and trapped for filtering, purification, or subsequent identification.

\subsection{Forms of dielectrophoresis}

Early DEP devices were typically composed of polydimethylsiloxane (PDMS) using photolithography methods to form microfluidic channels with metallic electrodes embedded within the PDMS. The PDMS structure was bonded to glass to form a DEP microfluidic device. The metallic electrode shape and placement played an important role in creating an electric field gradient. Although using metallic electrodes in direct contact with the sample successfully accomplished cell sorting at low voltage, problems of electrode fouling, increase in sample temperature, and spatial limitation existed. Rise in sample temperature is particular concern for biological samples as too high of temperatures can lead to cell death. Spatial limitations exist as a cell must come within approximately $30 \mu \mathrm{m}$ of the electrodes to experience the dielectrophoretic force because the electric field gradient drops off quickly moving away from the electrodes [11]. Spatial problems can be partially ameliorated by patterning electrodes to the top and bottom of the sample chamber or along the full height of the channel. However, this complicates

device fabrication and does not completely resolve the problem as narrowing channels for 
proximity sake decreases throughput. Alternative designs such as insulator-based DEP (iDEP) and contactless DEP (cDEP) among others have been implemented to successfully overcome common problems associated with earlier designs.

In iDEP, platinum electrodes are inserted at the inlet and outlet of the sample channel of a microfluidic device. The device is fabricated such that insulator structures at the center of the sample channel create a non-uniform electric field. As an electric potential is applied across the electrodes, the electric field must bend around the insulator structures creating a non-uniform electric field. Popular insulator structures include an array of pillars, a sawtooth design with teeth incrementally approaching each other, and partial obstructions. iDEP is advantageous over the use early designs as the gradient electric field spans from floor to ceiling of the sample channel, eliminating the spatial limitation associated with the use of traditional designs stated previously. Unfortunately, iDEP devices are still prone to problems such as electrolysis and Joule heating.

Contactless DEP (cDEP) is another form of DEP that eliminates problems commonly associated with DEP (spatial limitations, electrode fouling, Joule heating, and electrolysis). In cDEP, the sample and liquid electrode channels are isolated from each other by a thin $(\sim 20 \mu \mathrm{m})$ insulating barrier. At the center of the sample channel, insulator structures cause a non-uniform electric field in the same manner as described for iDEP devices. Using an AC electric source, the device works by means of capacitive coupling. Because cDEP devices have no contact between the electrodes and the sample channel, problems of electrode fouling and electrolysis are eliminated, while the gradient of the electric field is maintained from floor to ceiling. Due to the benefits of cDEP, it is well suited to analyze biological samples. 
Other forms of DEP exist (carbon-electrode DEP, light-induced DEP, "liquid electrode” DEP, reservoir-based DEP) but will not be addressed here as reviews are available in literature [11-15]. The DEP forms presented here provide enough context for the significance of this dissertation.

\section{Engineering significance}

This study stands apart from previous research of bacterial isolation and identification due to the unique contactless dielectrophoretic design and the potential impact the device could have on clinical diagnostics. Typical cDEP designs use PDMS to form microfluidic channels that leads to several problems in fabrication, operation, and reusability. The unique design presented addresses these problems and provides an avenue for simultaneous isolation, trapping, and identification of bacteria. As such, this unique design improves upon the current cDEP design and has the potential to decrease analysis time of bacteria from days to a matter of minutes.

The fabrication process of typical cDEP devices start with a silicon master mold that contains the microfluidic pattern created by deep reactive ion etching. Using this mold, PDMS is casted and cured to form the microfluidic channels. This casting process is often repeated many times as it is difficult to produce a consistent and strong seal of the thin $(\sim 20 \mu \mathrm{m})$ insulating barrier between the sample channel and the liquid electrodes. This inconsistency creates issues with repeatability from one device to the next. The use of PDMS also creates limitations for cDEP devices due to its dielectric breakdown. The thin insulating barrier must be as thin as possible to be able to generate an electric field. However, the thinner the barrier, the greater risk of surpassing the threshold for dielectric breakdown. 
The alternative cDEP design presented in this work addresses the problems commonly associated with cDEP devices. Instead of using PDMS, microfluidic channels are etched into a fused silica plate and PDMS is only used to seal the channels. Using fused silica eliminates the repeated PDMS casting and allows for a higher dielectric breakdown threshold. Using fused silica in lieu of PDMS also allows reproducibility in tests as well as reusability as the fused silica plate can be autoclaved between tests. Therefore, the unique design presented here overcomes problems of fabrication, operation, and reusability of cDEP devices.

The design presented here also allows for simultaneous isolation, trapping and identification of bacteria. As mentioned previously, the microfluidic channels are formed in fused silica. This material allows for acquisition of Raman spectra with minimal interference in wavenumber ranges of interest. This work presents the first cDEP-Raman device which isolates, traps, and identifies bacteria at the same time. Therefore, the unique cDEP-Raman design has great potential to decrease the analysis time of bacteria in a label-free way. 


\section{CHAPTER II}

\section{LITERATURE REVIEW}

\section{Raman spectroscopy for bacterial analysis}

A variety of label-free methods have been implemented to identify bacteria including laser-induced breakdown spectroscopy [16-18], Fourier transform infrared (FT-IR) spectroscopy [19,20], Raman spectroscopy [1,21], autofluorescence [22,23], and many others. Although each have their advantages, the use of Raman spectroscopy will be the focus of this research as it is a label-free technique, requires minimal sample preparation, is non-destructive, and is well suited for biological samples as water does not significantly impact Raman signatures.

The use of Raman spectroscopy to analyze whole bacteria started in the 1970s to address theories concerning vibrational states in biological membranes [24-28]. Over time, emphasis changed from theory to identification for medical applications. When considering spectral identification of bacteria, the differences in laboratory conditions to real world conditions must be accounted for. For example, culturing bacteria in laboratory settings is done with growth media favorable for the bacteria. In contrast, culture conditions of the human body vary from person to person. Researchers are aware of the discrepancy and have investigated the changes in Raman spectra due to factors such as temperature [29,30], growth phase [29-38], and growth media [29,30,33,38]. For the sake of this review, emphasis will be placed on the influence of growth media and phase on Raman spectra. The factor of temperature is ignored as temperature in the human body has a narrow range of values. 


\subsection{Influence of growth media on Raman spectra}

The influence of growth media on Raman spectra of bacteria has been investigated by a number of researchers $[29,30,33,39,40]$ with varied results. The majority of findings indicated differences in spectra due to growth media. For example, Mlynàrikovà et al. [39] compared Raman spectra of bacteria and yeast cultured in various growth media to identify a media that resulted in the least amount of spectral variance. Raman spectra were collected directly from colonies on agar plates. Using PCA groupings, the authors indicated spectral differences in bacteria according to growth media but did not study if those variations impacted bacteria classification.

In contrast, Premasiri et al. [41] found no differences as long as samples were properly washed. Sample preparation consisted of taking an aliquot of a liquid culture, centrifuging the sample to a small pellet, removing the supernatant, and adding fresh saline. Centrifuging and rinsing steps were repeated several times. Using PCA groupings, the authors showed how rinsing steps were required as media residue would influence spectral variance. Once media was removed after three washes, bacteria of the same species grown in different media were in the same PCA grouping.

\subsection{Influence of culture time on Raman spectra}

The influence of growth time or growth phase (metabolic state) on Raman spectra of bacteria has been studied by a number of researchers [29-38]. For example, Espagnon et al. [32] performed a classification study of 80 strains of bacteria and yeast using Raman spectra and multivariate statistical methods. Spectra were collected directly from colonies on agar plates after 6 and 24 hours of incubation to represent the exponential and stationary growth stages, respectively. Authors reported greater intensity of DNA and 
RNA related wavenumbers $\left(664,781,808,1095\right.$, and $\left.1569 \mathrm{~cm}^{-1}\right)$ for the log phase in comparison to the stationary phase.

Other articles have collected and compared Raman spectra of bacteria from different growth times, but have not specified growth stage. Choo-Smith et al. [34] collected and compared Raman spectra of bacterial colonies grown on agar plates for 6, 12, and 24 hours at various colony depths using a confocal Raman microscope. The authors reported greater spectral variation in colonies cultured for 12 to 24 hours as opposed to 6 hours. They concluded that shorter culturing times should be used for bacterial identification as these colonies were more homogenous.

Moritz et al. [36] investigated changes in the Raman spectra according to growth phase/metabolic state of the bacteria. Samples were grown in Luria-Bertani broth and growth state was identified by optical density (OD) measurements. Results indicated that within the log and transition phases, Raman peaks that are associated with RNA and DNA $\left(668,784,812,1100,1477\right.$, and $\left.1575 \mathrm{~cm}^{-1}\right)$ generally decrease over the lifespan of the bacteria, while peaks associated with proteins (852, 897, 934, 963, 1003, 1032, 1550 $\mathrm{cm}^{-1}$ ) increase. These trends are attributed to protein synthesis as bacteria experience a depletion of nutrients. Several protein peaks (1126, 1452, 1605, and $\left.1660 \mathrm{~cm}^{-1}\right)$ remained relatively consistent regardless of metabolic state. In the stationary phase, DNA and protein synthesis stops and is reflected in bacterial Raman signatures as peaks associated with RNA, DNA, and proteins generally remain consistent over time. The authors demonstrated discrimination of bacterial growth phase based on associated Raman spectra. 


\subsection{Influence of growth media and phase on classification of bacteria}

Although researchers have found differences in Raman spectra among culturing conditions, previous work demonstrates that bacteria can still be successfully identified by multivariate classification or chemometric methods [29,30,33,38]. For example, Harz et al. [30] found that varying culturing conditions such as media nutrients, temperature (30-37 ${ }^{\circ} \mathrm{C}$ ), and culture age (6-72 hours) indeed influenced the resulting spectra. Older samples were reported to have a smaller signal-to-noise ratio for single cell analysis. In addition, wavenumbers in the $1575 \mathrm{~cm}^{-1}$ region, tentatively assigned to the deformation vibration of an amide, decreased over time. These changes had minimal effect on the classification of bacteria.

Huang et al. [38] also found variations in Raman spectra according to growth phase, reporting that wavenumbers associated with RNA to protein (783-785, 1230-1295 $\left.\mathrm{cm}^{-1}\right)$, lipids $\left(1062 \mathrm{~cm}^{-1}\right)$ and carbohydrates $\left(544-553 \mathrm{~cm}^{-1}\right)$ were higher at the log phase than at stationary phase. In contrast, amides among wavenumbers $1650-1680 \mathrm{~cm}^{-1}$ were higher in the stationary phase than in the log phase and are attributed to growth-phase related changes in membrane compounds, polysaccharides, proteins, lipids, and nucleic acids. The differences between growth phases did not hinder the overall species discrimination.

Hutsebaut et al. [29] studied how growth media, time (24-48 hours), and temperature $\left(30-37{ }^{\circ} \mathrm{C}\right)$ influenced the classification of 30 bacillus strains using Raman spectra, linear discriminant analysis, and principal component analysis. Colonies were grown on various agar media (brain heart infusion, tryptone soya agar, and gelatin agar) and smeared directly onto a $\mathrm{CaF}_{2}$ substrate for Raman analysis. The authors reported a 
species classification accuracy of $92.34 \%$ when varying all parameters. Although growth time ranged from 1-2 days, it is unclear what growth phases were included in the study.

Xie et al. [35] used confocal laser tweezers Raman spectroscopy (LTRS) to determine the effects of bacterial synchronization and growth phase on discrimination between six different species of bacteria. Bacteria were cultured in LB liquid media and harvested at various stages of growth for analysis, with growth stages being identified according to OD measurements. Authors reported that bands 783, 811, 1099, and 1578 $\mathrm{cm}^{-1}$ increase between lag and log phase suggesting DNA and RNA synthesis, while bands 723, 783, and $1578 \mathrm{~cm}^{-1}$ associated with RNA are larger at the log phase than in the stationary phase. However, high discrimination was still achieved between species even for unsynchronized and randomly assigned growth phases with the use of general discrimination analysis.

Research has also been conducted on bacteria to determine Raman spectral differences due to growth phase, media, and excitation wavelength. Kunapareddy et al. [33] studied several different Gram-positive and Gram-negative bacteria using excitation wavelengths between 200-260 nm to develop two-dimensional Raman signatures. They found slight changes in two-dimensional spectra due to culture medium and growth phase with greater variations due to growth phase. A common change regardless of whether the bacteria were Gram-positive of Gram-negative was the peak intensity ratio between 1485 $\mathrm{cm}^{-1}$ (adenine and guanine bases) and $1616 \mathrm{~cm}^{-1}$ (tyrosine and tryptophan). The ratio was larger for bacteria in the log phase or when using an excitation wavelength of $248 \mathrm{~nm}$ as opposed to $232 \mathrm{~nm}$. The increase in nucleic acids is expected during the log phase as the bacteria are actively dividing. The higher peak ratio of $1485 \mathrm{~cm}^{-1} / 1616 \mathrm{~cm}^{-1}$ when 
varying laser excitation is attributed to the resonant Raman response where certain molecules experience increased Raman scattering when excitation wavelengths approach their electronic transitions. For example, Raman spectra from laser wavelengths in the ultraviolet region like $244 \mathrm{~nm}$ and $229 \mathrm{~nm}$ will primarily be rich in information about nucleic acids and aromatic amino acids, respectively [42]. Despite the spectral variations, Kunapareddy et al. [33] found that bacteria could be distinguished regardless of growth phase or culture medium.

\subsection{Raman studies of Mycobacterium}

Very few studies have investigated changes in Raman spectra of Mycobacterium due to culture conditions and growth stage. Some related research has been conducted using other methods. For example, varying the culture conditions of Mycobacterium paratuberculosis has been shown to influence acid resistance and protein expression [43]. Another study used matrix-assisted laser desorption/ionization-time of flight mass spectrometry (MALDI-TOF MS) to find differences in Mycobacterium phlei and Mycobacterium smegmatis [44]. It should be noted that MALDI-TOF MS requires a considerable amount of sample preparation including rupturing bacteria cell walls.

Stöckel et al. [37] studied the differences in Raman spectra of Mycobacterium aurum (pigmented) and M. smegmatis (non-pigmented) according to growth phase. For the case of M. aurum, the Raman spectrum is heavily influenced by carotenoids, a class of hydrocarbons (terpenes) responsible for its pigmentation. Associated peaks appear at 1518, 1189-1127, and $1005 \mathrm{~cm}^{-1}$. In the stationary and death phases, band position and relative band intensity for carotenoid-related peaks change. For example, the $1127 \mathrm{~cm}^{-1}$ band increases in intensity in comparison to the $1158 \mathrm{~cm}^{-1}$ band as the bacteria matures. 
For the non-pigmented M. smegmatis, peaks associated with mycolic acids from mycobacteria's cell envelope are more prominent $\left(1081,1305,1446\right.$, and $\left.1748 \mathrm{~cm}^{-1}\right)$ especially upon reaching the stationary phase.

Kumar et al. [45] found that varying carbon sources among glucose, glycerol, and acetate for M. smegmatis resulted in varied Raman intensities for carotenoid-related peaks during the exponential phase. Specifically, glucose sources increased carotenoid peaks with wavenumbers 1156 and $1524 \mathrm{~cm}^{-1}$.

Literature contains plenty of articles concerning Raman spectroscopy and Mycobacterium. However, most of the research is focused on identification [1,46-49] and characterization of physical properties [50], cellular extracts [51], enzymes [52,53], and proteins [54]. However, only few articles [37,45] focus on how culture conditions for Mycobacterium influence variations in Raman spectra and resulting classification. Although many research articles are available concerning Raman spectroscopy and Mycobacterium, there is still a need for research concerning the influence of culture conditions of Mycobacterium on Raman spectra.

\section{DEP and Raman Spectroscopy for Bacterial Analysis}

Raman spectroscopy of clinical samples (blood, saliva, sputum, urine, etc.) require an isolation step. As such, samples must be pretreated prior to analysis to remove debris. Most label-free techniques to isolate and concentrate bacteria according to species involve the use of microfluidic devices. Within these devices, several mechanisms of sorting can be employed such as hydrodynamic and electrical methods. Of these techniques, DEP stands apart as a label free technique which isolates cells by capitalizing on physical and electrical characteristics of target cells. 
Dielectrophoresis is the use of non-uniform electric fields to cause motion in particles due to the magnitude of the applied electric field, gradient of the electric field, and properties of the particle (shape, size, homogeneity, electrical permittivity, etc.). Although dielectrophoresis was introduced in the early 1950s [55], its use in various research fields remained fairly dormant until the 1990s when techniques such as photolithography assisted in the fabrication of minute structures like microfluidic devices [11]. This advancement in fabrication techniques was crucial for DEP as it drastically dropped voltage requirements due to proximity of electrodes to cells within a sample, thus creating a much more realistic means for sample sorting. As a result, the use of DEP in microfluidic devices as well as the methods of implementation have significantly increased.

Many articles concerning isolation, trapping, and concentration of bacteria using DEP exist. For example, DEP has been used to separate bacteria from water [56-58], erythrocytes [59-61], yeast [62-67], and sample debris [68-74]. Bacteria has also been separated from other bacteria based on differing genera [61,62,71,72,75,76], species [57], and serotypes [77]. Other researchers have used DEP as a means to separate or identify bacteria according to viability $[68,78,79]$ and antibiotic resistance [80-84]. In some cases, DEP has been used to concentrate bacteria to a specific area for subsequent analysis or identification [60,61,71,85-87].

To meet the need for isolation and identification, DEP is coupled with other techniques such as impedance analysis (DEPIA) or Raman spectroscopy (DEP-Raman spectroscopy). DEPIA has been used to concentrate and quantify bacteria [88-91]. The device can also be equipped with immunoglobulins for detection and identification as a 
bacteria binds to an associated immunoglobulin resulting in a change in impendence [89]. This identification scheme is prone to false positives due to non-specific binding. In addition, the method requires the use of metallic electrodes, which exposes the technique to common problems associated with DEP as mentioned previously.

An example of a DEP-Raman involves the use of a quadruple electrode arrangement to concentrate bacteria by negative DEP for Raman analysis [92-94]. Although successful, the design appears impractical as it is meant for small sample volumes $(\sim 200 \mu \mathrm{L})$ with some of the studies injecting even smaller volumes $(10 \mu \mathrm{L})$ of concentrated bacteria at the DEP site for successful demonstration [92,93]. Not only is the sample size problematic, but the design is prone to common DEP-related issues of electrode fouling, electrolysis, and Joule heating. In addition, the quadruple electrode design is ill-suited to analyze samples containing debris or more than one bacteria at a time. Other examples of DEP-Raman spectroscopy include sample labeling using Raman reporters or antibodies [95,96]. The use of labels increases costs, limits shelf life, and may result in wasted materials due to the broad range of bacteria strains that can be present in a sample. Label-free identification methods are appealing to cut costs, increase simplicity, and reduce the risk of false positives. In previous cases where DEP-Raman spectroscopy systems did not use labels or tags, the device was made using traditional metallic electrodes in contact with the sample channel [61,97], exposing the device to standard DEP problems of electrode fouling and electrolysis.

DEP has great potential for trapping of bacteria for subsequent or simultaneous identification. Currently, DEP devices that provide simultaneous trapping and identification of bacteria fall short of clinical application due to practicality, shelf life, or 
potential sample damage during operation. There is a need to develop a prompt bacterial identification method that is accurate, reliable, and applicable for clinical settings. Although DEP has great potential, there are still hurdles to overcome such as electrode fouling, electrolysis, and sample size limitations. In this dissertation, a contactless DEPRaman device is proposed, fabricated, and tested to meet the need of simultaneous trapping and identification of bacteria while avoiding common issues associated with DEP devices.

\section{Motivation and Goals}

Traditional bacterial analysis methods rely on visual identification of large bacteria colonies requiring one to two days for fast growing bacteria. In the case of slow growing bacteria such as mycobacteria, this process can take weeks. This lengthy identification time is problematic and many researchers have worked on solving this problem as documented in the previous sections. To analyze bacteria in a clinical sample faster than traditional methods, bacteria need to be isolated from other sample debris prior to identification.

The goal of this work is to decrease the analysis time of bacteria by designing, building, and fabricating a device for simultaneous isolation and identification. It is hypothesized that a cDEP-Raman device can isolate bacteria from debris and identify bacteria on the same platform. To this end, the following specific aims, categorized under identification and isolation, were addressed:

1. Identification

a. Determine if Raman spectra of bacteria changes according to growth media and phase 
b. Evaluate the best multivariate statistical method for classification

c. Determine the best way to implement PCA centering and scaling

d. Assess the best PC selection method

2. Isolation

a. Develop a cDEP design to eliminate common problems associated with the technique and allow for simultaneous identification

b. Model cDEP design to isolate debris from bacteria

c. Build and test cDEP-Raman device to separate bacteria from debris and collect Raman spectra of trapped bacteria

This dissertation documents the development of a cDEP-Raman spectroscopy device that improves operating parameters, addresses fabrication issues associated with cDEP, and allows for simultaneous DEP trapping and identification by Raman spectroscopy. Thus, the design offers label-free sorting and simultaneous identification that may lead to a decrease in the analysis time of bacteria. 


\section{CHAPTER III}

\section{EFFECT OF PRINCIPAL COMPONENT ANALYSIS CENTERING AND SCALING}

\section{ON CLASSIFICATION OF MYCOBACTERIA FROM RAMAN SPECTRA ${ }^{1}$ [14]}

\section{Abstract}

Raman spectroscopy has been used for decades to detect and identify biological substances as it provides specific molecular information. Spectra collected from biological samples are often complex, requiring the aid of data truncation techniques such as principal component analysis (PCA) and multivariate classification methods. Classification results depend on the proper selection of principal components (PCs) and how PCA is performed (scaling and/or centering). There are also guidelines for choosing the optimal number of PCs such as a scree plot, Kaiser criterion, or cumulative percent variance. The goal of this research is to evaluate these methods for best implementation of PCA and PC selection to classify Raman spectra of bacteria. Raman spectra of three different isolates of mycobacteria (Mycobacterium sp. JLS, Mycobacterium sp. KMS, Mycobacterium sp. MCS) were collected and then passed through PCA and linear discriminant analysis for classification. Principal component analysis implementation as well as PC selection was evaluated by comparing the highest possible classification accuracies against accuracies determined by PC selection methods for each centering and scaling option. Centered and unscaled data provided the best results when selecting PCs based on cumulative percent variance.

\footnotetext{
${ }^{1}$ This paper was published in Applied Spectroscopy and coauthored by Elizabeth Vargis and Michael Sieverts.
} 


\section{Introduction}

Standard methods to detect and identify bacteria take one to two days for fastgrowing bacteria. In the case of slow-growing bacteria such as Mycobacterium, the analysis process can take weeks to complete. This lengthy analysis time increases costs to patients and hospitals especially when the patient must be quarantined until lab results are available. In order to decrease bacterial analysis time and the expenses associated with it, researchers have turned to a variety of laser spectroscopy methods to identify bacteria.

Raman spectroscopy is well suited for bacterial analysis as there is very little sample preparation resulting in a decreased analysis time for detection. In addition, it provides a rich amount of information concerning molecular structure. The spectral result is often referred to as a Raman signature and can be used to identify bacteria. However, spectra of bacteria are complex, making it difficult to distinguish between species and strains when examining the spectra alone. Along with Raman spectroscopy, other methods generate complex spectra such as Fourier transform infrared spectroscopy (FTIR) $[34,98,99]$ and surface-enhanced Raman spectroscopy $[61,71,94,100]$. Due to the complex nature of the spectra, many researchers ease data processing by truncating data using principal component analysis (PCA) prior to classifying bacteria [34,92,101-106].

A detailed description of PCA is omitted here as many sources provide a thorough explanation of PCA and its associated mathematical reasoning [107,108]. Briefly, PCA truncates data by rotational reorientation to maximize variance along the new axis while preserving the relationship and order among the data points. The axis with the greatest amount of variance becomes the first principal component (PC). The second PC is orthogonal to the first and maximizes the remaining variance. Each subsequent 
component is orthogonal to the previous PCs, accounting for incrementally less variance of the data. The PCs can be used for further classification as they retain the information from the original data. While forming the PCs, there are a number of ways in which data are treated: scaled and centered, scaled and not centered, centered and not scaled, or not centered or scaled. Scaling is typically used for data sets which have drastically different values or units from one variable to the next. During scaling, the correlation matrix is used rather than the covariant matrix. Centering means shifting the coordinates such that the center of the data lies at zero. The process of scaling and centering is referred to as normalizing the data. It distributes the weight of each variable equally and can influence the selection of PCs and resulting classification. Another factor that affects classification is PC selection such as a scree plot, the Kaiser criterion, or cumulative percent variance (CPV) [107-109]. Here, these guidelines and the effect of PCA centering and scaling were evaluated based on the resulting spectral classification accuracy when identifying Mycobacterium sp. JLS [110], Mycobacterium sp. KMS [111], and Mycobacterium sp. MCS [112] with Raman spectroscopy.

\section{Materials and Methods}

\subsection{Bacteria growth and sample preparation}

Mycobacterium sp. JLS, Mycobacterium sp. KMS, and Mycobacterium sp. MCS were taken from stock solutions preserved in 15\% glycerol and 85\% Lysogeny broth (LB) at $-80^{\circ} \mathrm{C}$. From the stock, bacteria was inoculated in sterilized LB composed of Bacto Tryptone (BD Biosciences, San Jose, CA, USA), Bacto Yeast Extract (BD Biosciences, San Jose, CA, USA), and sodium chloride (Thermo Fisher Scientific, Chicago, IL, USA) in a 2:1:2 ratio, respectively, with $10 \mathrm{~g}$ of Bacto Tryptone per liter of 
water. Bacteria were grown in LB media at $36^{\circ} \mathrm{C}$ under constant shaking of 220 rotations/min. Growth times were in the range of 4-8 days to incorporate differences in cell membrane composition during the lifetime of the bacteria as other studies have indicated spectral variations due to the age of the bacteria [31,34,102,113]. Samples were prepared such that there were four biological replicates for each species. Three of the biological repetitions were used to create technical replicates (3, 2, and 2 for each species). The technical replicates were used to capture spectral variance due to culture age, independent of inoculation concentration.

Samples were prepared from the LB media by placing $1 \mathrm{~mL}$ of inoculated broth into a sterile $1.5 \mathrm{~mL}$ centrifuge tube. The solution was centrifuged for $2 \mathrm{~min}$ at $6000 \mathrm{rpm}$ using a Thermo Scientific Heraeus Pico 21 microcentrifuge. The supernatant was then discarded and the bacteria pellet formed at the bottom was resuspended in a sterile $0.85 \%$ saline solution and vortexed to ensure proper mixing. This centrifuging process was performed three times. From the final solution, $5 \mu \mathrm{L}$ was extracted for Raman analysis. To avoid interference from glass microscope slides, aluminum tape was placed on a glass microscope slide to create a platform for the sample. Upon adding the sample, a quartz coverslip was placed on top of the bacteria solution to prevent contact between the sample and the microscope objective.

\subsection{Raman spectroscopy}

Raman spectra of the mycobacteria were collected using a Renishaw inVia Raman microscope equipped with a $633 \mathrm{~nm}$ laser. Using a 50× Leica objective, a group of bacteria was identified and put into focus. As the laser spot size is approximately 2 $\mu \mathrm{m}$, each spectra was acquired from 1-6 bacteria cells. Spectra were collected using a 
laser power of approximately $7 \mathrm{~mW}, 50 \mu \mathrm{m}$ spectrometer slit, and an 1,800 groove/mm grating. Each spectrum was accumulated from six scans, each with a 10-s acquisition time over a wavenumber range of $900-1800 \mathrm{~cm}^{-1}$. Renishaw Wire 4.1 software was used for cosmic ray removal and baseline correction. Spectra smoothing and normalization was performed using Spekwin32 [114], an open source software. Normalization set the highest peak within the wavenumber range equal to 1 and scaled all other intensities accordingly. Therefore, some Raman intensity values were negative after normalization.

\subsection{Statistical methods}

The software, R, was used to perform PCA and linear discriminant analysis (LDA). Spectral data were prepared for analysis by converting data to comma-separated values (csv) files and compiling all data into a master data set from which the training and test data sets were formed. The variables for the master data set were wavenumbers in the range of $900-1800 \mathrm{~cm}^{-1}$ with step sizes in the range of $0.85-0.90 \mathrm{~cm}^{-1}$ totaling 979 variables. In total, there were 237 spectra with 79 spectra representing each Mycobacterium strain. Spectra were organized according to biological and technical repetitions. Two of the four biological repetitions were assigned to the test and training data set. Therefore, the training and test data sets were selected to be independent of each other. Pairing the four biological repetitions creates six possible combinations. All combinations were formed and analyzed. The distribution of each Mycobacterium strain to the test and training data sets is displayed in Table 3.1. It should be noted that LDA was the only classification method used for subsequent analysis as it consistently provided better results in preliminary testing when compared against quadratic 
Table 3.1. Assignment of spectra from each mycobacteria (Mycobacterium sp.

JLS, Mycobacterium sp. KMS, and Mycobacterium sp. MCS) to form the test and training data sets.

\begin{tabular}{ccccccccc} 
& \multicolumn{2}{c}{ JLS } & \multicolumn{2}{c}{ KMS } & \multicolumn{2}{c}{ MCS } & \multicolumn{2}{c}{ Total } \\
\cline { 2 - 9 } Data set & Train & Test & Train & Test & Train & Test & Train & Test \\
\hline 1 & 50 & 29 & 49 & 30 & 50 & 29 & 149 & 88 \\
2 & 49 & 30 & 49 & 30 & 49 & 30 & 147 & 90 \\
3 & 40 & 39 & 39 & 40 & 40 & 39 & 119 & 118 \\
4 & 39 & 40 & 40 & 39 & 39 & 40 & 118 & 119 \\
5 & 30 & 49 & 30 & 49 & 30 & 49 & 90 & 147 \\
6 & 29 & 50 & 30 & 49 & 29 & 50 & 88 & 149 \\
\hline
\end{tabular}

discriminant analysis, classification trees, random forests, support vector machines, gradient boosted trees, and k-nearest neighbor (data not shown).

\section{Results and Discussion}

\subsection{Raman spectroscopy}

The quality of spectra collected depends on several factors including the number of bacteria present and image resolution. As spectra were collected using a nonmotorized stage, focusing differed from one sample to the next. In addition, the concentration of the bacteria also varied but was approximately $0.1 \mathrm{mg} / \mu \mathrm{L}$. To eliminate spectral differences due to focusing, concentration, and background noise, spectra underwent post-processing. Figure 3.1 illustrates the process, including the original (a), baseline corrected (b), and normalized (c) spectra. Figure 3.1d is composed of a spectrum from each bacteria overlaid on top of each other. 


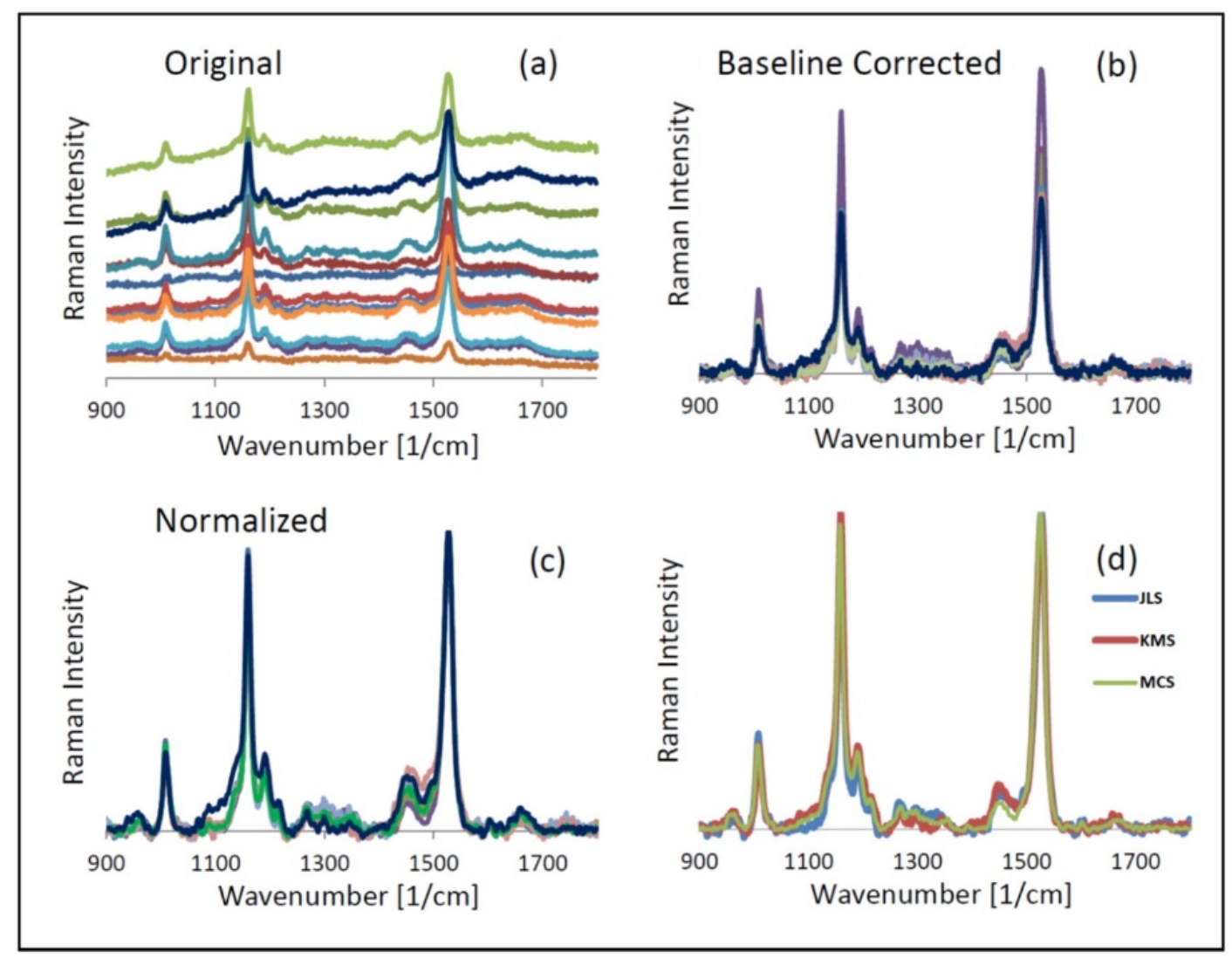

Figure 3.1. Examples of the original spectra (a), baseline corrected spectra (b), normalized spectra (c), and spectra from each mycobacteria collected with a 10s acquisition time and six accumulations (d). Spectra were taken of Mycobacterium sp. JLS, Mycobacterium sp. KMS, and Mycobacterium sp. MCS.

\subsection{Influence of Centering and Scaling of PCA on Classification Results}

Scaling the data in $\mathrm{R}$ is equivalent to using the correlation matrix to perform PCA. The correlation matrix is typically used when the variables are expressed in different units and this discrepancy between variables needs to be minimized. Thus, the values need to be scaled such that the variables have equal weight. The covariance matrix is used when the data are not scaled, typically the case for variables that are of comparable units. The data sets used in this study are in units of Raman intensity (Figure 3.1). As 
such, the use of the covariance matrix (not scaling) is expected to produce better results as opposed to the correlation matrix.

Unlike scaling, centering does not change the variance. Instead, the data are shifted such that the mean lies at zero while retaining the variance. The choice of centering will affect the scores for each associated PC and will also influence the total number of PCs. In general, it is advised to center the data when performing PCA [115]. It is anticipated that centering will not significantly change classification results for data sets that are not scaled.

To determine the effect of centering and scaling on high dimensional, collinear data sets with more variables than observations, the highest bacterial classification accuracies were found for each centering and scaling combination. This was done by determining the classification accuracy resulting from each PC and then identifying the highest classification accuracy. This accuracy was used as a benchmark for each method and is referred to as the highest or maximum classification accuracy throughout the text. To ensure the trends in accuracies are not a result of the spectra selection, all six data set combinations were used as a means of cross-validation. The highest classification accuracies for each PC, scaling and centering option, and data set was determined. The results are displayed in Figure 3.2. Data which were scaled and uncentered during PCA resulted in the lowest classification accuracies for almost all data sets. Centering and scaling the data typically provided results between the lowest and highest for all data sets. Data sets which were not scaled performed almost identical to each other. For further analysis of PC selection methods, data set 3 was used as it provided the highest classification accuracy with the most common trend among the data sets: scaled and 


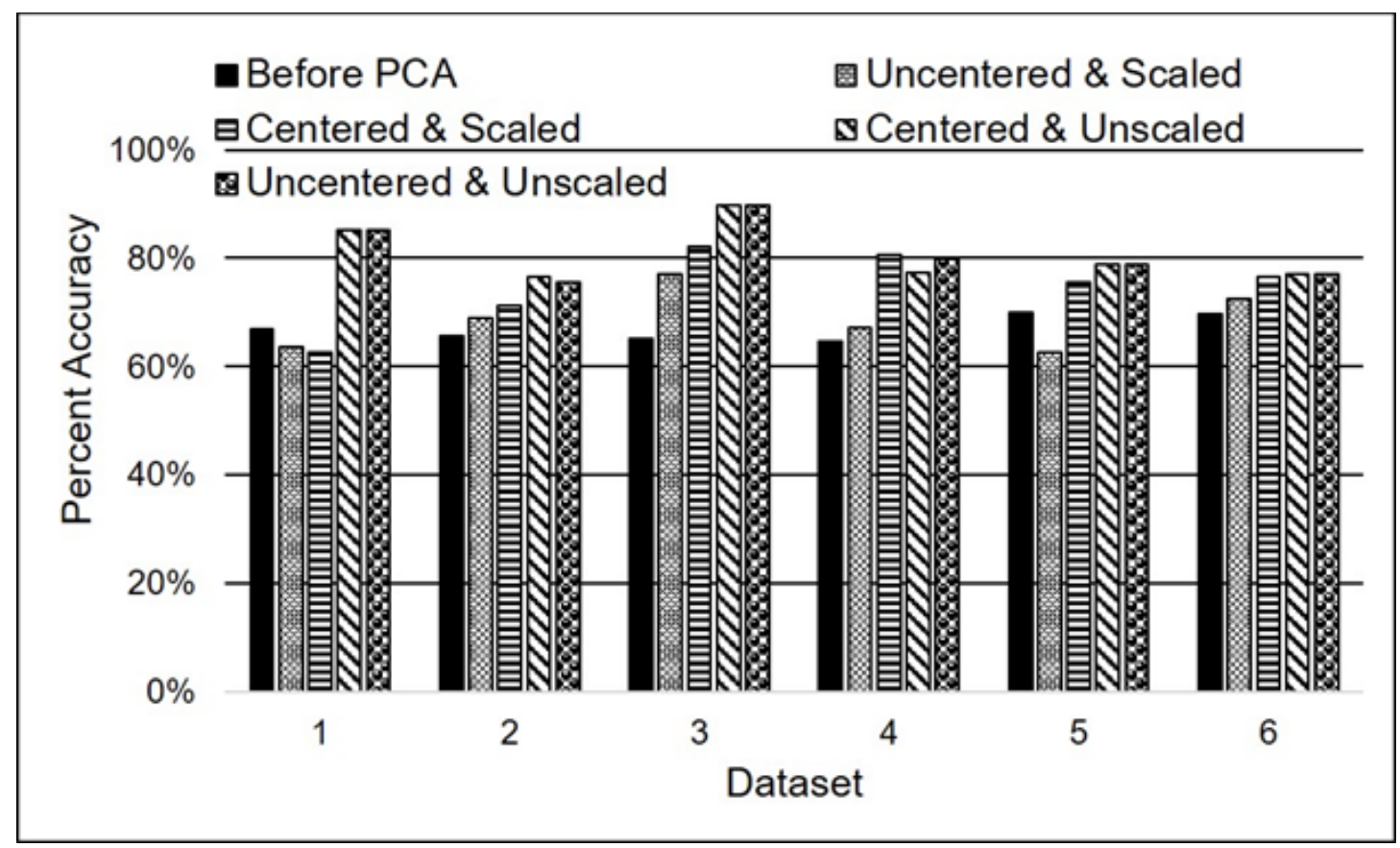

Figure 3.2. The highest classification accuracies possible for data which were centered and scaled, uncentered and scaled, centered and unscaled, and uncentered and unscaled during PCA.

uncentered data had the lowest accuracy while data which were unscaled had equivalent or highest accuracies.

\subsection{Selection of PCs}

Figure 3.2 illustrates the classification accuracies before and after performing PCA. In most cases, the classification accuracy improved. Literature also provides examples of PCA improving classification accuracies of high dimensional data sets [116]. Although performing PCA can improve results, finding the appropriate number of PCs to incorporate can be difficult especially for data sets involving Raman spectra. Typically, optimal PCs are identified using a scree plot, the Kaiser criterion, or cumulative percent variance (CPV). Each method was explored and resulting accuracies were compared to each other. 


\subsubsection{Selection of PCs based on the scree plot}

Scree plots graph the variance (eigenvalues) of the data according to PCs and are commonly used to identify the number of principal components to implement for future classification. The suggested number of PCs is identified by a sudden break or change in variance from one PC to the next. For simplicity, the break will be referred to as the elbow. Figure 3.3 shows scree plots from data set 3 according to the four different ways in which PCA was implemented: scaled and centered, scaled and uncentered, centered and unscaled, and uncentered and unscaled. Three of the four have much more pronounced elbows at either two or three PCs. The centered and unscaled data set had the most unique trend in shape, scale of variance, and suggested number of PCs. Scree plots were constructed for all data sets to identify the suggested number of PCs to use.

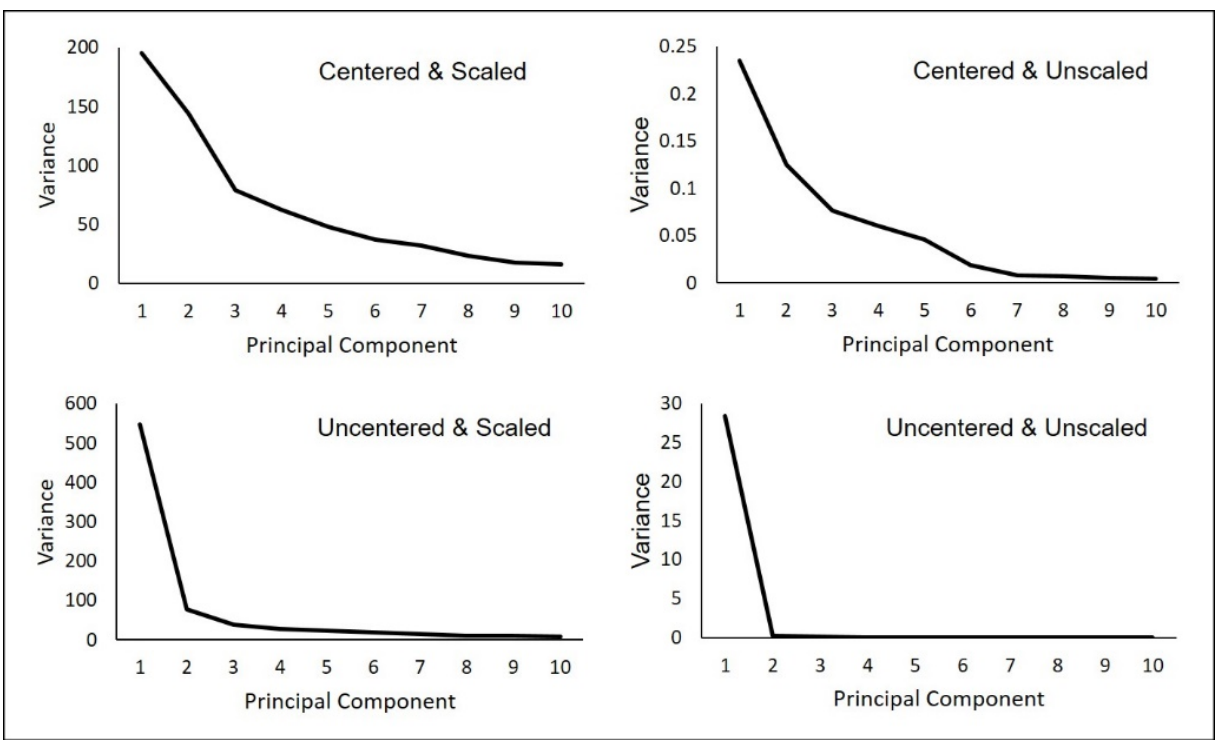

Figure 3.3. Examples of scree plots from data set 3 which were scaled and centered, scaled and uncentered, unscaled and centered, and unscaled and uncentered during PCA. Note the varying scales of variances in the y-axis for each condition. 
Using the scree plot method, PCs were identified for classification and the resulting classification accuracies were compared to the maximum classification accuracies for each data set (previously identified, Figure 3.2). The average drop in classification accuracy using the scree plot method can be found in Figure 3.4 with error bars indicating standard deviation. It is evident that the scree plot method is the worst method of the three to select PCs for classification. The secondary axis of Figure 3.4 includes the best classification accuracies and their associated standard deviation for each centering and scaling option.

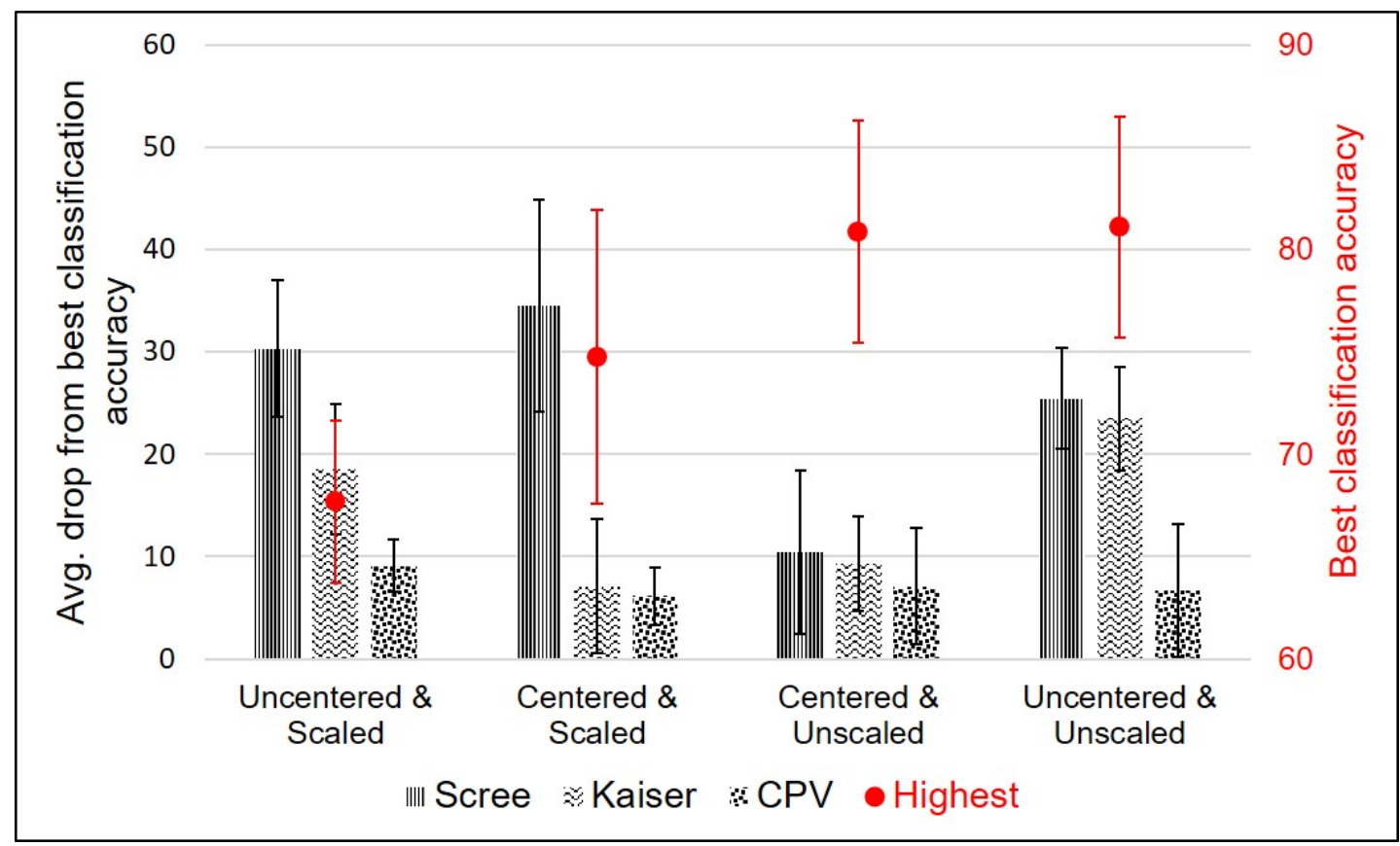

Figure 3.4. Average drop in classification accuracy from the maximum accuracy possible for choosing PCs based on the scree plot method, Kaiser method, and cumulative percent variance (CPV) covered by the PCs. The secondary axis in red indicate the best classification accuracies (averaged over six data sets) with error bars indicating standard deviation. 


\subsubsection{Selection of PCs based on the Kaiser method}

The Kaiser (also known as Guttman-Kaiser) criterion suggests using PCs that have associated eigenvalues which are greater than the average eigenvalue. The Jolliffe rule is a modification to the Kaiser criterion which suggests a lower cutoff value of 0.7 times the average eigenvalue $[107,109]$. Suggested PCs were identified by the Kaiser and Joliffe rules and their associated accuracies were determined. This classification accuracy was compared to the maximum classification accuracy for each data set and scaling and centering option to determine the drop in classification accuracy, which is displayed in Figure 3.4 with error bars indicating the standard deviation. Figure 3.4 indicates that in comparison to the scree plot method, the Kaiser method improved classification results for all scaling and centering options.

\subsubsection{Selection of PCs based on cumulative percent variance}

Another method to determine which PCs to use is by choosing PCs based on their associated CPV. For example, a common method for PC selection is to use the PCs which account for $95 \%$ of the variance. However, this method has its disadvantages. Previous studies [116] have shown how the number of PCs used affects classification error among several different classification methods for high dimensional data such as Raman spectra. For all classification methods studied in the article, the classification error initially decreased. The subsequent behavior was dependent on the classification method, but the methods either remained at roughly the same error rate or increased in error with the use of more PCs. A similar behavior was found by plotting classification accuracy for data set 3 as a function of PCs, as illustrated in Figure 3.5. The classification 
accuracies have a sharp increase at approximately five PCs and a sharp decrease in accuracy with the last few PCs.

Overall, data sets which were scaled resulted in lower classification accuracies as illustrated by Figure 3.4, indicating that not all variables should have equal weight in classification. Centering did not have a significant influence on the highest classification results as long as data were not scaled. The scree plot method for PC selection performed better for unscaled data, while the Kaiser method performed better for centered data came closer to the best accuracy. The cumulative percent variance did not appear to be influenced by either scaling or centering.

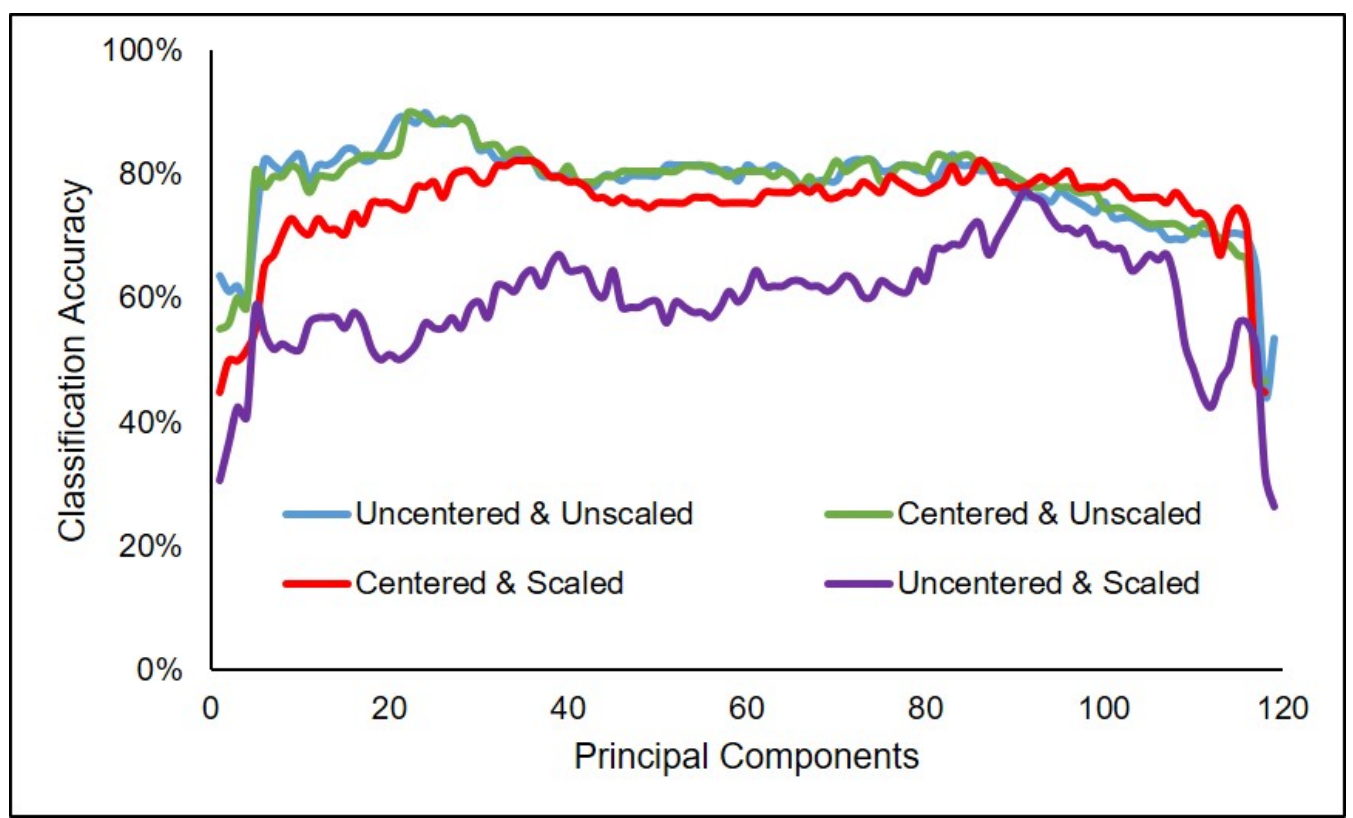

Figure 3.5. Linear discriminant analysis classification accuracy as a function of PCs for all centering and scaling options from data set 3. 
One question that may arise is whether these classification accuracies are applicable to a larger range of conditions such as differing growth times and introducing more species. In this study, different growth times were accounted for by using bacteria grown for 4-8 days with a series of biological and technical repetitions. For pigmented bacteria, as is the case for the Mycobacterium isolates (JLS, MCS, and KMS), researchers have used peaks assigned to carotenoids (1150 and $1520 \mathrm{~cm}^{-1}$ ) to identify different species of mycobacteria from each other [46]. However, it has been found that carotenoid levels of mycobacteria can vary depending on the growth stage, with the stationary phase providing a reproducible trend in Raman signatures over time [37]. Future studies will need to take this into account.

When Raman spectra from additional classes are included, a drop in classification accuracy is expected. The classification accuracies presented here are obtained using Raman spectra from three very similar strains. The genomic makeup for Mycobacterium sp. MCS is 97\% symmetrically identical to Mycobacterium sp. KMS [117]. As illustrated in Figure 3.6, plotting three PCs against each other indicates that the three strains can be distinguished. However, the differences are slight. Adding more strains, species, or genera could change the classification accuracies by lumping the data of the three strains into one area. As such, spectra of bacteria would need to go through a series of classification stages to allow for both broad and specific identification. 


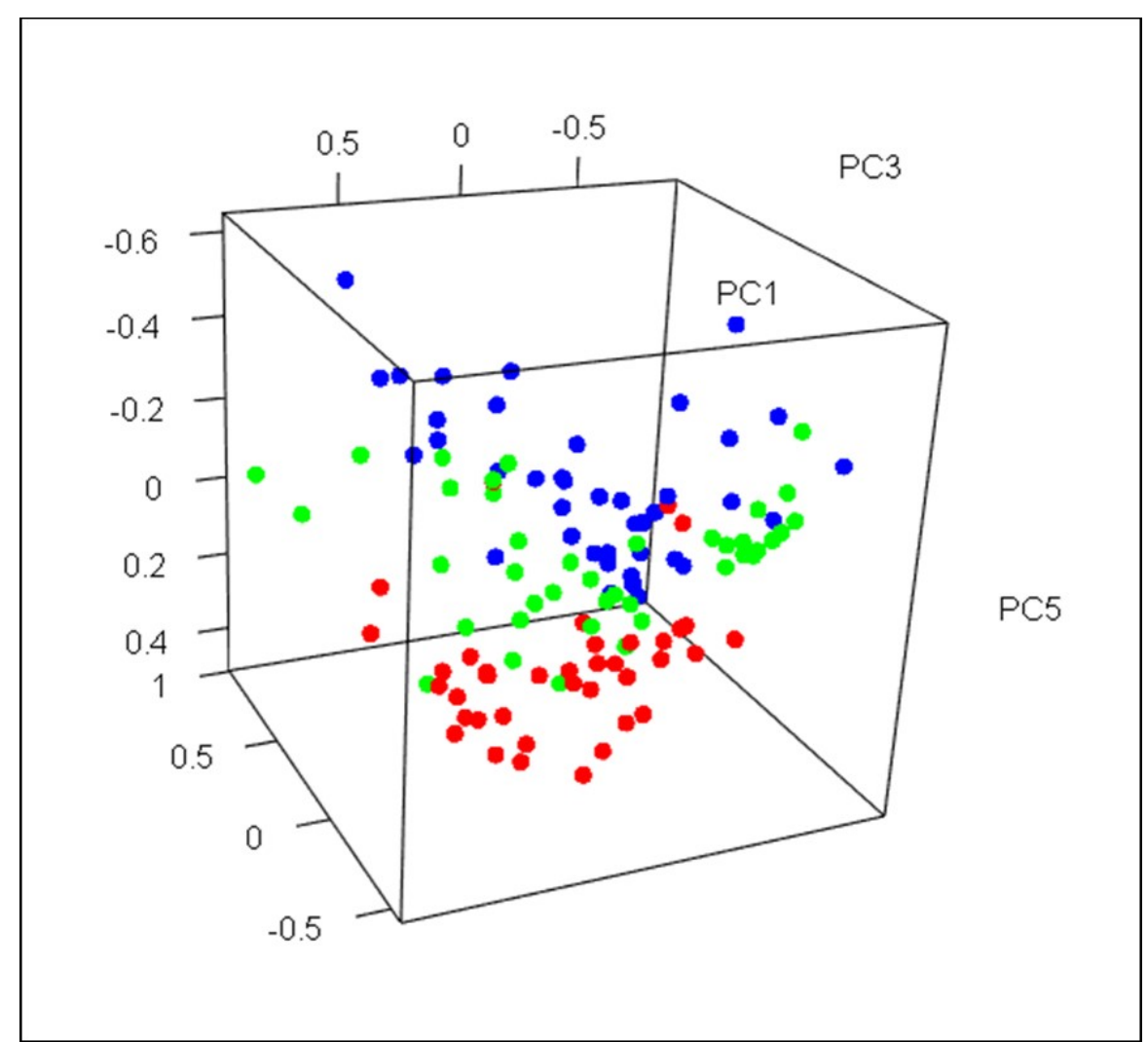

Figure 3.6. Plot of PCs 1, 3, and 5 from data set 3 which was centered and unscaled. Blue, green, and red dots represent Mycobacterium sp. JLS, Mycobacterium sp. MCS, and Mycobacterium sp. KMS respectively.

\section{Conclusions}

In an effort to streamline and reduce bacterial analysis time, PCA and LDA is being used to spectrally classify mycobacteria. In this study, Raman spectra of three different strains of Mycobacterium were passed through LDA to determine the effect of classification accuracies before and after PCA. In almost all cases, PCA improved classification accuracies by tens of percentage points. Centering and scaling options during PCA were investigated to determine how future data sets should be treated. Investigating these methods was done by comparing classification accuracies after performing PCA and LDA and selecting PCs based on the scree plot method, Kaiser 
method, and cumulative percent variance. Scaled and uncentered data provided the worst classification accuracies, while unscaling the data provided the highest accuracy at $90 \%$. The Kaiser and Jolliffe rules were superior to the scree plot method for all scaling and centering options. When comparing accuracy results using the Kaiser and Jolliffe rules as well as the CPV, centering and scaling the data has the smallest drop in accuracy from the best classification accuracy. However, centering and scaling did not provide the highest classification accuracies. Centered and unscaled data had the highest classification accuracy with all PC selection methods providing reasonable results. Future studies will use centered and unscaled data when performing PCA and select PCs that cover $94.4 \%$ of the variance. 


\section{CHAPTER IV}

\section{INFLUENCE OF GROWTH MEDIA AND PHASE ON RAMAN SPECTRA OF}

\section{MYCOBACTERIA}

\section{Abstract}

When developing a Raman spectral library to identify bacteria, differences between laboratory conditions and real-world conditions must be considered. For example, culturing bacteria in laboratory settings is performed with specific conditions tailored to the bacteria such as nutrient content, $\mathrm{pH}$, agitation, or growth temperature. In contrast, culture conditions in the human body may not have the ideal set of circumstances for bacteria to grow. To address these differences, the effect of environmental conditions such as temperature, $\mathrm{pH}$, growth media, and growth phase on Raman spectra has been tested. However, the majority of the research has focused on Gram-positive or Gram-negative bacteria. This article focuses on the influence of growth media and phase on Raman spectra of Mycobacterium sp. MCS, an acid-fast bacteria. Results showed that spectral differences in growth phase and media can be distinguished by direct spectral observation and multivariate analysis. Results were comparable to those found in literature for other types of bacteria, such as Gram-positive, Gram-negative, and acid-fast bacteria. The results confirmed the need for spectral libraries to account for an array of culture conditions.

\section{Introduction}

A variety of label-free methods have been implemented to identify bacteria including laser-induced breakdown spectroscopy [16-18], Fourier transform infrared 
spectroscopy [19,20], Raman spectroscopy [1,21], and autofluorescence [22,23].

Although each have their advantages, the use of Raman spectroscopy will be the focus of this research as it is a non-destructive, label-free technique that requires minimal sample preparation. Plus, it is well suited for biological samples as water does not significantly impact Raman signatures.

Raman spectroscopy is sensitive to small changes in bacteria cells due to growth media [29,30,33,39,40,45]. For example, Mlynàrikovà et al. [39] compared Raman spectra of bacteria and yeast cultured in various growth media to identify a media which lead to the least amount of spectral variance. Raman spectra were collected directly from colonies on agar plates. Using principal component analysis (PCA), spectral differences in bacteria were found according to growth media.

Other researchers have seen no differences in Raman spectra according to media as long as the bacteria are properly washed [41]. Sample preparation consisted of taking an aliquot of a liquid culture, centrifuging the sample to a small pellet, removing the supernatant, and adding fresh saline. Centrifuging and rinsing steps were repeated several times. Using PCA groupings, the authors showed how rinsing steps were required as media residue would influence spectral variance. Once media was removed after three washes, bacteria of the same species grown in different media were in the same PCA grouping.

The influence of growth phase on Raman spectra has also been researched and includes direct spectral observation as well as statistical discriminatory methods [2938,118,119]. Cell phases include lag, log, transition, stationary, and death phase. During the lag phase, the bacteria adjust to their surroundings and little growth occurs. The log 
phase is characterized by cells that are actively growing and dividing at a consistent rate. During the transition phase, cell growth slows down but is greater than the rate of cell death. The stationary phase occurs when the rates of cell growth and death are equal, and the death phase is where the rate of cell death is greater than cell growth.

Moritz et al. [36] investigated the changes in Raman spectra according to the metabolic state of bacteria. Samples were grown in Luria-Bertani broth and growth phase was identified by optical density (OD) measurements. The majority of Raman peaks associated with RNA and DNA decreased as bacteria aged from the log to the stationary phase, which has been seen by many groups [30-33,35,38,118,119]. Moritz et al.[36] also found that protein-related peaks increase or stay the same over the same timeframe. Other sources concerning Raman spectra of protein peaks for bacteria indicate mixed results $[33,38,118,119]$. Regardless of the specific result, literature indicates differences in Raman spectra of bacteria according to growth phase whether by observation or by statistical analysis.

Of the studies cited above, only two have investigated changes in Raman spectra of mycobacteria due to culture conditions and growth phase [37,45]. Stöckel et al. [37] studied the differences in Raman spectra of Mycobacterium aurum (pigmented) and $M$. smegmatis (non-pigmented) according to growth stage. For M. aurum, Raman spectra are heavily influenced by carotenoids, an organic pigment containing eight isoprene molecules. Peaks associated with carotenoids appear at 1005, 1127-1189, and $1518 \mathrm{~cm}^{-1}$. In stationary and death phases, band position and relative band intensity for carotenoidrelated peaks change. In Stöckel's article, the $1127 \mathrm{~cm}^{-1}$ band increased in intensity compared to the $1158 \mathrm{~cm}^{-1}$ band as the bacteria matured. In addition, band position of 
carotenoid peaks ranging from $1500-1550 \mathrm{~cm}^{-1}$ varied depending on the conjugated chain length of the carotenoid. For M. smegmatis, Raman spectra are dominated by peaks associated with mycolic acids from the cell envelope (1081, 1305, 1446, and $1748 \mathrm{~cm}^{-1}$ ) especially upon reaching the stationary phase. Mycobacteria respond similarly to changes in growth media. Kumar et al. [45] found that varying carbon sources among glucose, glycerol, and acetate for $M$. smegmatis resulted in varied Raman intensities for carotenoid-related peaks during the exponential phase.

There are many articles documenting the effects of growth media and phase on Raman spectra of Gram-positive and Gram-negative bacteria. In comparison, there are very few articles concerning the effect of growth media and phase on Raman spectra of acid-fast bacteria, like mycobacteria. The prompt and accurate diagnosis of mycobacteria is of interest globally, especially Mycobacterium tuberculosis, one of the bacterial species responsible for tuberculosis. According to the 2017 Global Tuberculosis report from the World Health Organization [120], tuberculosis is one of the top ten causes of death worldwide. Despite the need to research mycobacteria, there are only a few articles that cover the influence of growth media and phase on Raman spectra of mycobacteria.

The purpose of this article is to identify how growth media and phase influence Raman spectra of Mycobacterium sp. MCS. Using linear discriminant analysis (LDA), spectral differences were found according to phase (log and stationary) and culture media (Lysogeny broth, Brain Heart Infusion, Middlebrook, and Kirchner). Results are in general agreement with literature, where peaks associated with carotenoids increase and peaks associated with DNA/RNA and mycolic acids decrease as bacteria ages from the log phase to the stationary phase. 


\section{Materials and Methods}

\subsection{Bacteria Culturing}

Mycobacterium sp. MCS was taken from a stock solution preserved in 15\%

glycerol and $85 \%$ Lysogeny broth (LB) at $-80{ }^{\circ} \mathrm{C}$ until cultured in sterilized liquid media. The four culture media used were LB, Brain Heart Infusion (BHI), Middlebrook, and Kirchner media. Middlebrook and Kirchner media were used as they are nutrient specific for mycobacteria, while BHI and LB are not. Thereby, data could be collected from bacteria grown in ideal and nonideal conditions.

Table 4.1 indicates the composition of all media added to water to make 1 liter of media. Ingredients for LB and BHI media were dissolved directly in 1 liter of media, separated into $125 \mathrm{~mL}$ Erlenmeyer flasks with approximately $50 \mathrm{~mL}$ aliquots, and sterilized by autoclaving. Middlebrook and Kirchner were made by adding all components, except for enrichment media and serum, to water to make $900 \mathrm{mLs}$ of solution. After dissolving, the solution was divided into $40 \mathrm{~mL}$ aliquots in $125 \mathrm{~mL}$ Erlenmeyer flasks and autoclaved. Before inoculating the media with mycobacteria, 10 mLs of enrichment or serum was aseptically added to each Erlenmeyer flask. Bacteria were grown in each liquid media at $36{ }^{\circ} \mathrm{C}$ under constant shaking of 220 rotations/min. The log, transition, and stationary growth phases were identified using optical density (OD) measurements at $580 \mathrm{~nm}$. OD measurements were taken using a BioTek (Winooski, VT, USA) Synergy 2 Multi Mode Reader and Gen 5.1.11 software. 
Table 4.1. Ingredient list for Lysogeny Broth, Brain Heart Infusion, Middlebrook, and

Kirchner media. Contents are mixed with distilled water to make 1 liter of media.

\begin{tabular}{|c|c|c|c|}
\hline Media & Component & Amount & Supplier \\
\hline \multirow{3}{*}{ Lysogeny Broth } & Bacto Tryptone & $10 \mathrm{~g}$ & BD Biosciences (San Jose, CA) \\
\hline & Bacto Yeast Extract & $5 \mathrm{~g}$ & BD Biosciences (San Jose, CA) \\
\hline & Sodium Chloride & $10 \mathrm{~g}$ & Thermo Fisher Scientific (Waltham, MA) \\
\hline Brain Heart Infusion & Brain Heart Infusion Broth & $37 \mathrm{~g}$ & Sigma-Aldrich (St. Louis, MO) \\
\hline \multirow{3}{*}{ Middlebrook } & Middlebrook 7H9 dehydrated media & $4.7 \mathrm{~g}$ & BD Difco (Sparks, MD) \\
\hline & Middlebrook ADC enrichment & $100 \mathrm{~mL}$ & BD Difco (Sparks, MD) \\
\hline & Glycerol & $2 \mathrm{~mL}$ & Avantor (Center Valley, PA) \\
\hline \multirow{8}{*}{ Kirchner } & $\mathrm{Na}_{2} \mathrm{HPO}_{4} \cdot 12 \mathrm{H}_{2} \mathrm{O}$ & $19 \mathrm{~g}$ & Acros Organics (Morris Plains, NJ) \\
\hline & $\mathrm{KH}_{2} \mathrm{PO}_{4}$ & $2 \mathrm{~g}$ & Alfa Aesar (Tewksbury, MA) \\
\hline & $\mathrm{MgSO}_{4} \cdot 7 \mathrm{H}_{2} \mathrm{O}$ & $0.6 \mathrm{~g}$ & Alfa Aesar (Tewksbury, MA) \\
\hline & $\mathrm{Na}_{3} \mathrm{C}_{6} \mathrm{H}_{5} \mathrm{O}_{7} \cdot 2 \mathrm{H}_{2} \mathrm{O}$ & $2.5 \mathrm{~g}$ & Fisher Scientific (Hampton, NH) \\
\hline & L-asparagine & $5 \mathrm{~g}$ & Alfa Aesar (Tewksbury, MA) \\
\hline & Glyercol & $20 \mathrm{~mL}$ & Avantor (Center Valley, PA) \\
\hline & Phenol red & $0.0012 \mathrm{~g}$ & Acros Organics (Morris Plains, NJ) \\
\hline & Inactviated horse serum & $100 \mathrm{~mL}$ & Life Technologies (Carlsbad, CA) \\
\hline
\end{tabular}

Samples were prepared from culture media by placing $1 \mathrm{~mL}$ of inoculated broth into a microcentrifuge tube. The broth was centrifuged for $2 \mathrm{~min}$ at $6000 \mathrm{rpm}$ using a Heraeus Pico 21 microcentrifuge (Thermo Fisher Scientific, Waltham, MA, USA). The supernatant was removed and the bacteria pellet was resuspended in $0.85 \%$ saline solution and vortexed for proper mixing. This rinsing procedure was performed three times to remove media residue. From the final solution, $5 \mu \mathrm{L}$ was extracted, and dried on top of an aluminum-covered microscope slide. Upon drying, Raman spectra were collected.

\subsection{Raman spectra}

Raman spectra of Mycobacterium sp. MCS were collected using an inVia Raman microscope (Renishaw, Wotton-under-Edge, United Kingdom) with a 633 nm laser, 50 x Leica objective, and an 1800 groove/mm grating. Spectra were acquired using a laser power of $14 \mathrm{~mW}$ with a 10-s exposure time and three accumulations. Collected spectra 
had a wavenumber range of 200-3200 $\mathrm{cm}^{-1}$. Renishaw Wire 4.1 software was used for baseline correction and cosmic ray removal. Spekwin32 was used for normalization.

\subsection{Statistical methods}

The open access software, $\mathrm{R}$, was used to perform linear discriminant analysis (LDA) on spectral data to determine if differences in growth phase (log and stationary) and growth media (LB, BHI, Middlebrook, and Kirchner) could be distinguished. Three biological samples for each media were prepared and four spectra (technical replicates) were collected at each phase for a total of 96 spectra. Wavenumbers ranged from 200$3200 \mathrm{~cm}^{-1}$ with step sizes ranging from $0.852-0.853 \mathrm{~cm}^{-1}$ totaling 3522 variables.

\section{Results and Discussion}

\subsection{Discrimination based on growth phase}

Mycobacterium sp. MCS was grown in LB, BHI, Middlebrook, and Kirchner media with growth phase identified by plotting OD measurements as indicated by Figure 4.1. Raman spectra were acquired from bacteria in the log and stationary phases. Twelve spectra for each phase and media were collected, formatted, and discriminated based on growth phase using LDA. The resulting confusion matrix and LDA plot is presented in Table 4.2 and Figure 4.2, respectively. Classification results were $94.8 \%$ accurate with only 5 spectra out of 96 being misclassified. These results indicate that there are indeed discernable spectral differences between the three growth phases regardless of culture media. 


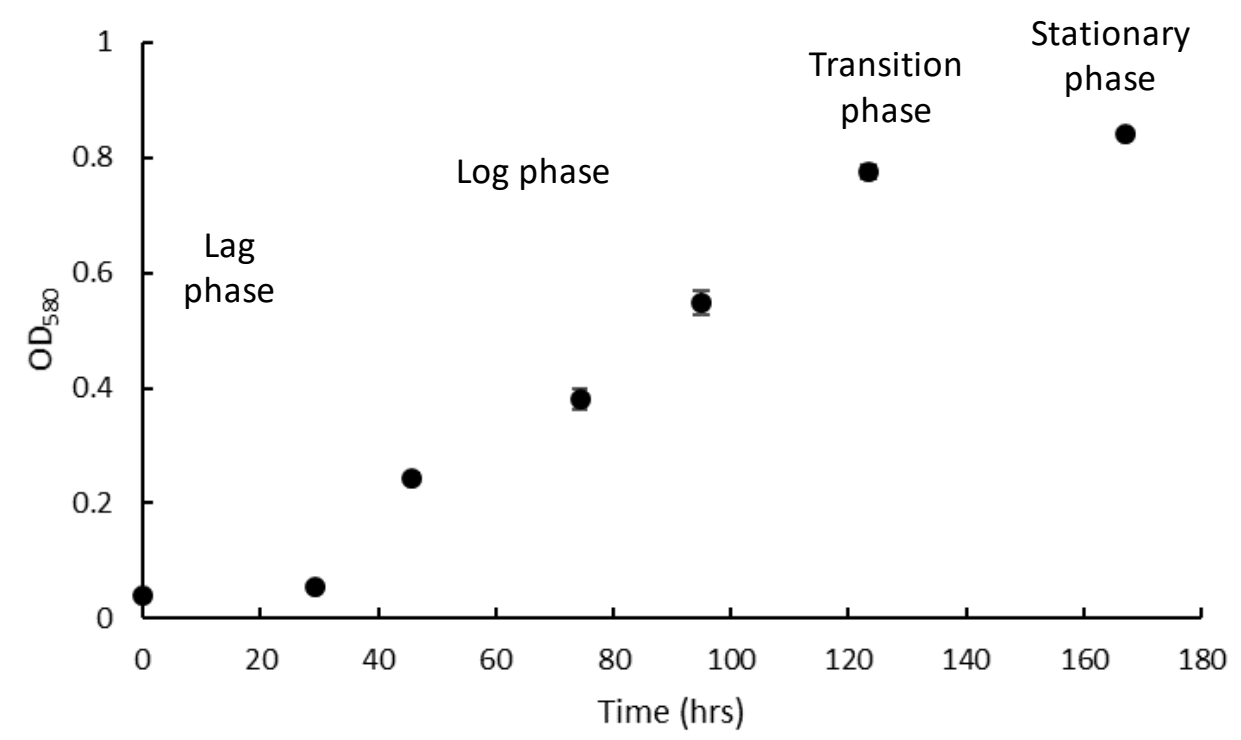

Figure 4.1. Growth curve for Mycobacterium sp. MCS in LB media based on optical density measurements at $580 \mathrm{~nm}$. Error bars at each point represent the standard deviation among seven technical replicates.

Table 4.2. Confusion matrix for LDA classification of Mycobacterium sp. MCS according to growth phase (log and stationary) and cultured in LB, BHI, Middlebrook, and Kirchner media. Classification results in 94.8\% accuracy.

\begin{tabular}{|c|cc|}
\hline TruelPredicted & Log & Stationary \\
\hline Log & 46 & 2 \\
Stationary & 3 & 45 \\
\hline
\end{tabular}




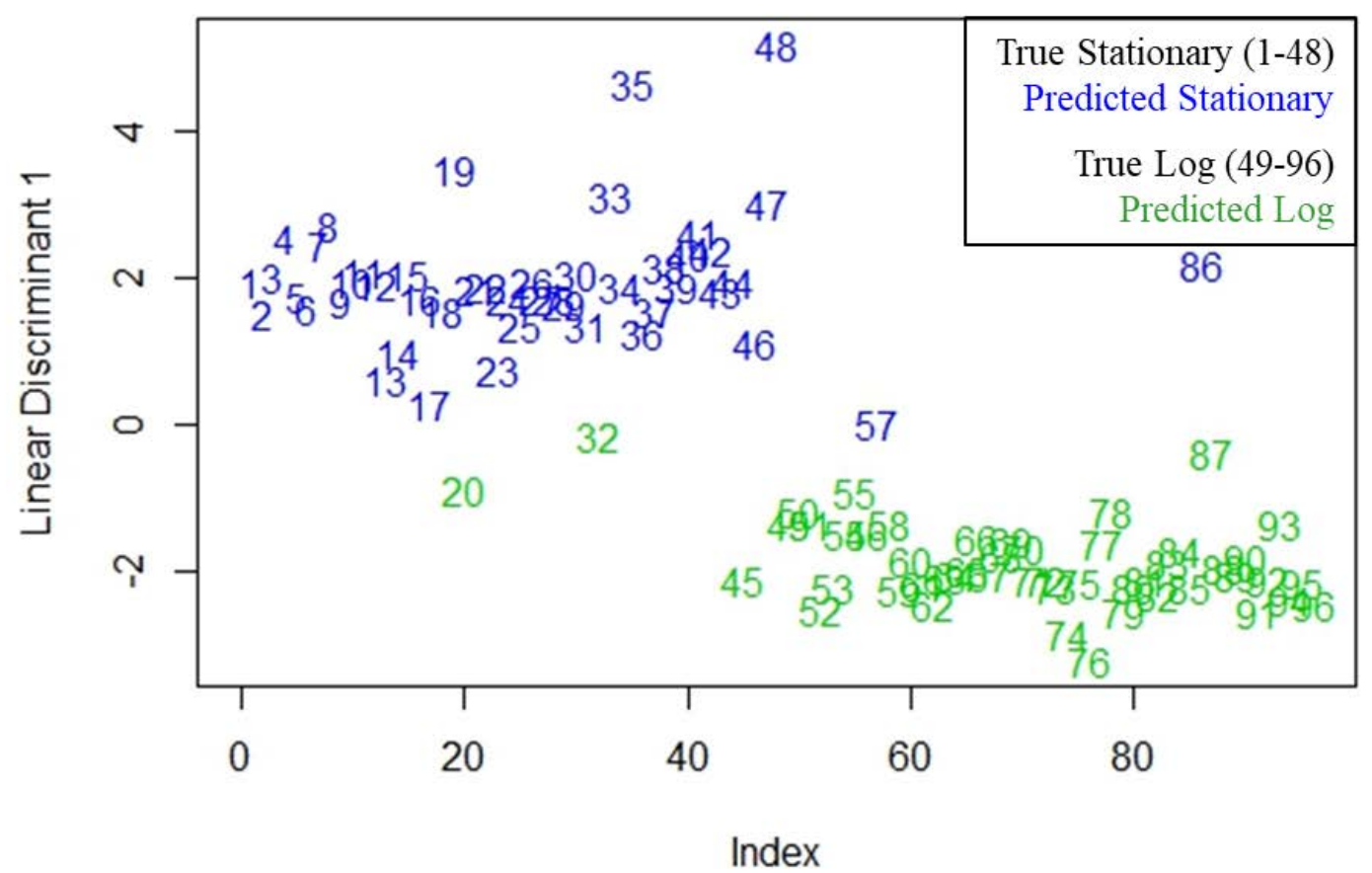

Figure 4.2. Linear discriminant analysis plot of spectra from Mycobacterium sp. MCS at the stationary (index 1-48) and log (index 49-96) phases. Blue and green text indicates the predicted stationary and log phases, respectively. Classification results in $94.8 \%$ accuracy.

\subsection{Discrimination based on growth media}

Mycobacterium sp. MCS colonies were cultured in LB, BHI, Middlebrook, and Kirchner media and the growth phase was monitored by performing OD measurements. Upon reaching the log and stationary phases, Raman spectra were collected. Twelve spectra were collected for each phase and media combination. The spectra were analyzed using LDA and classified according to culture media. The resulting confusion matrices are presented in Table 4.3 and the LDA plots for the log and stationary phases are displayed in Figure 4.3 and 4.4, respectively. Classification accuracies were $85.4 \%$ for the log phase and $93.8 \%$ for the stationary phases. As observed in Figure 4.4, spectra 
collected from bacteria in the stationary phase had the clearest separation according to media. Discrimination of media was more difficult in the log phase as seen in Figure 4.3. These results indicate that there are indeed discernable spectral differences between bacteria grown in different media and is in agreement with many literature sources $[29,30,33,39,40]$.

The classifications documented in Table 4.3 are more easily understood when observing Figure 4.3 and Figure 4.4. For example, Table 4.3 indicates there was one spectrum from Kirchner media that was classified as Middlebrook in the log phase. This can be seen in Figure 4.3 by the blue diamond. It is clearly far removed from the rest of the Kirchner data points (blue circles). Likewise, in the stationary phase, there was one Middlebrook spectrum that was classified Kirchner. This spectrum can be identified in Figure 4.4 by the black circle grouped closely by all the Kirchner data points (blue circles).

Table 4.3. Confusion matrices for LDA classification Mycobacterium sp. MCS grown in Lysogeny Broth (LB), Brain Heart Infusion (BHI), Kirchner (K), and Middlebrook (M) media. Matrices are grouped according to log and stationary phases, which have classification accuracies of $85.4 \%$ and $93.8 \%$, respectively.

\begin{tabular}{|c|cccc|cccc|}
\multicolumn{10}{c|}{ Log phase } & Stationary phase \\
\hline TruelPredicted & BHI & K & LB & M & BHI & K & LB & M \\
\hline BHI & 11 & 0 & 1 & 0 & 11 & 0 & 1 & 0 \\
K & 0 & 11 & 0 & 1 & 0 & 12 & 0 & 0 \\
LB & 0 & 1 & 10 & 1 & 1 & 0 & 11 & 0 \\
M & 2 & 0 & 1 & 9 & 0 & 1 & 0 & 11 \\
\hline
\end{tabular}




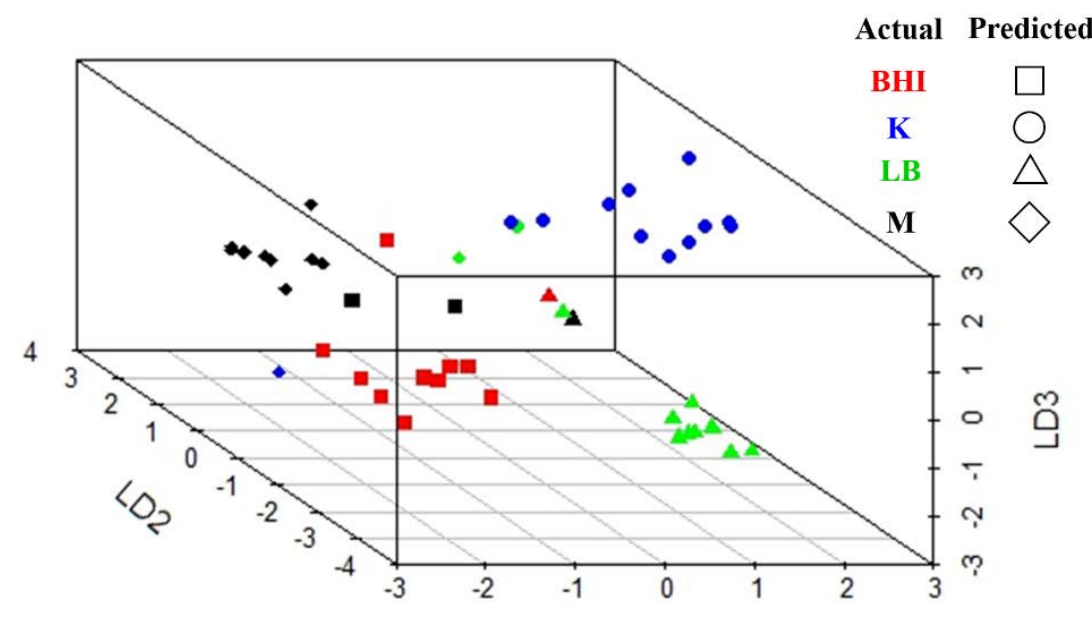

LD1

Figure 4.3. Plot of linear discriminants of Raman spectra of Mycobacterium sp. MCS at the log phase according to culture media. Media included Brain Heart Infusion (BHI), Lysogeny broth (LB), Middlebrook (M), and Kirchner (K). The plot displays the actual media by color and the predicted media by shape as indicated by the legend. Classification resulted in $85.4 \%$ accuracy.

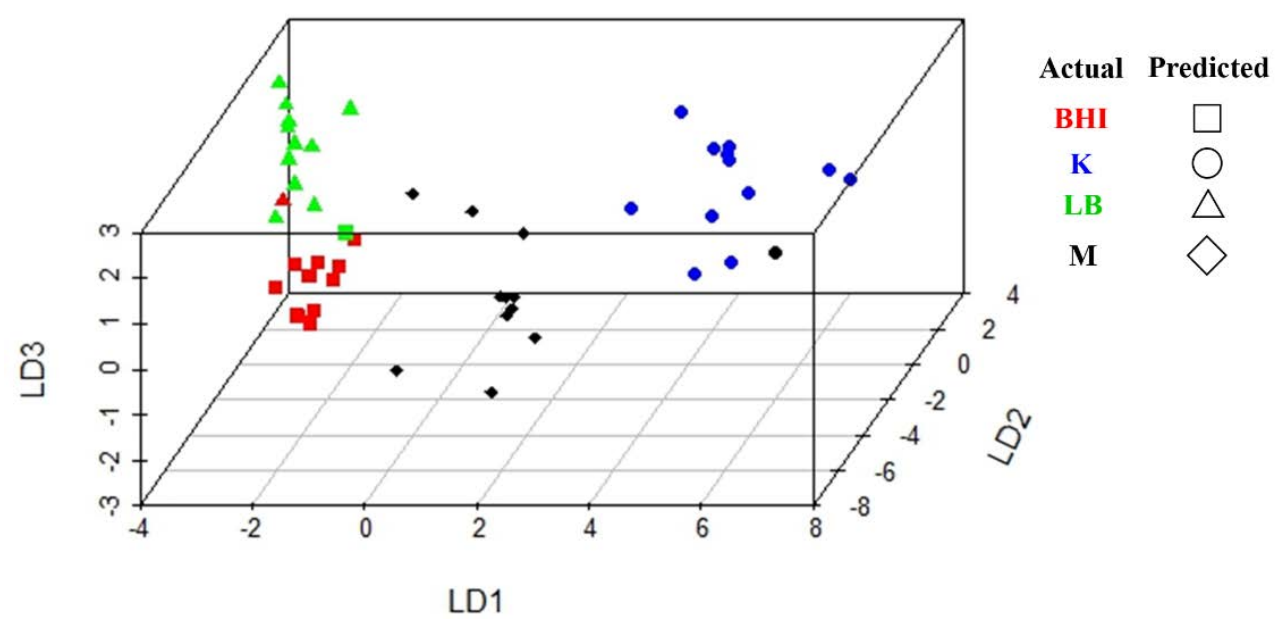

Figure 4.4. Plot of linear discriminants of Raman spectra of Mycobacterium sp. MCS at the stationary phase according to culture media. Media included Brain Heart Infusion (BHI), Lysogeny broth (LB), Middlebrook (M), and Kirchner (K). The plot indicates the actual media by color and the predicted media by shape as indicated by the legend. Classification resulted in 93.8\% accuracy. 


\subsection{Spectral differences due to culture conditions}

Spectra of Mycobacterium sp. MCS were collected at the log and stationary

phases for all media (Figure 4.5 and Figure 4.6) to identify trends and compare the results to literature. Literature indicates that the majority of Raman peaks associated with DNA and RNA decrease as bacteria age from the log to the stationary phase for Gram-positive or Gram-negative bacteria [30,32,33,35,36,38,100,118,119] while the majority of protein peaks increase or remain unchanged [36]. These results are supported by the work of Talukder et al. [121] who used a quantitative Western immunoblot to determine concentrations of DNA and proteins of E. coli cells through the log and stationary phases. They found that protein to DNA ratio increased as E. coli (Gram-negative) aged.

This trend in DNA was compared to spectra collected from Mycobacterium sp. MCS. Of the DNA peaks observed from Mycobacterium sp. MCS, the $782 \mathrm{~cm}^{-1}$ peak was the most intense and clearly distinguishable. The peak generally followed the expected trend of decreasing as the bacteria aged from the log to the stationary phase. The only exception was for bacteria grown in BHI media where it had a minimal increase in peak intensity.

The spectral differences may be attributed to varying DNA/RNA associated with the specific functions of cells during log and stationary phases. During log phase, cells are actively dividing by binary fission. Prior to division, the cells have twice as much DNA. The concentration of DNA/RNA-regulating proteins also varies depending on growth phase. For example, Fis (factor for inversion) is a DNA-binding protein that plays a role in DNA recombination and replication. Hfq (host factor for phage $\mathrm{Q} \beta$ ) protein regulates specific mRNA translation [122,123] and DNA repairs [123]. HU proteins 
influence DNA replication, recombination, transposition, and repair [124-128]. During the exponential phase, Fis, Hfq, and HU are at their highest concentrations and are most prominent among nucleoid proteins $[121,129]$. In contrast, the role of the stationary phase becomes that of survival and is characterized by elevated levels of Dps [121,129], a starvation-induced DNA-binding protein. Dps causes the DNA to become more compact forming a biocrystal to protect chromosomal DNA and aides in survival during long-term stressors [130]. It should be noted that Dps concentration in the late stationary phase is higher than Fis, Hfq, and HU combined in the log phase. Log and stationary phases are characterized by specific cell functions and physical characteristics. These changes in cell function and physiology provide an explanation for the trends seen in Raman spectra where DNA/RNA- and protein-related peaks decrease and increase respectively as bacteria age from the log to stationary phase.

As described, changes in Raman DNA-related peaks for Mycobacterium appear to follow the same trend. However, DNA-related peaks are not prominent in spectra collected for Mycobacterium sp. MCS. The peaks observed in Figures 4.5 and 4.6 are heavily influenced by carotenoids and mycolic acids specific to Mycobacterium sp. MCS. Therefore, discussion of spectral trends over the lifetime of the bacteria should also include carotenoids and mycolic acids specific to mycobacteria. 


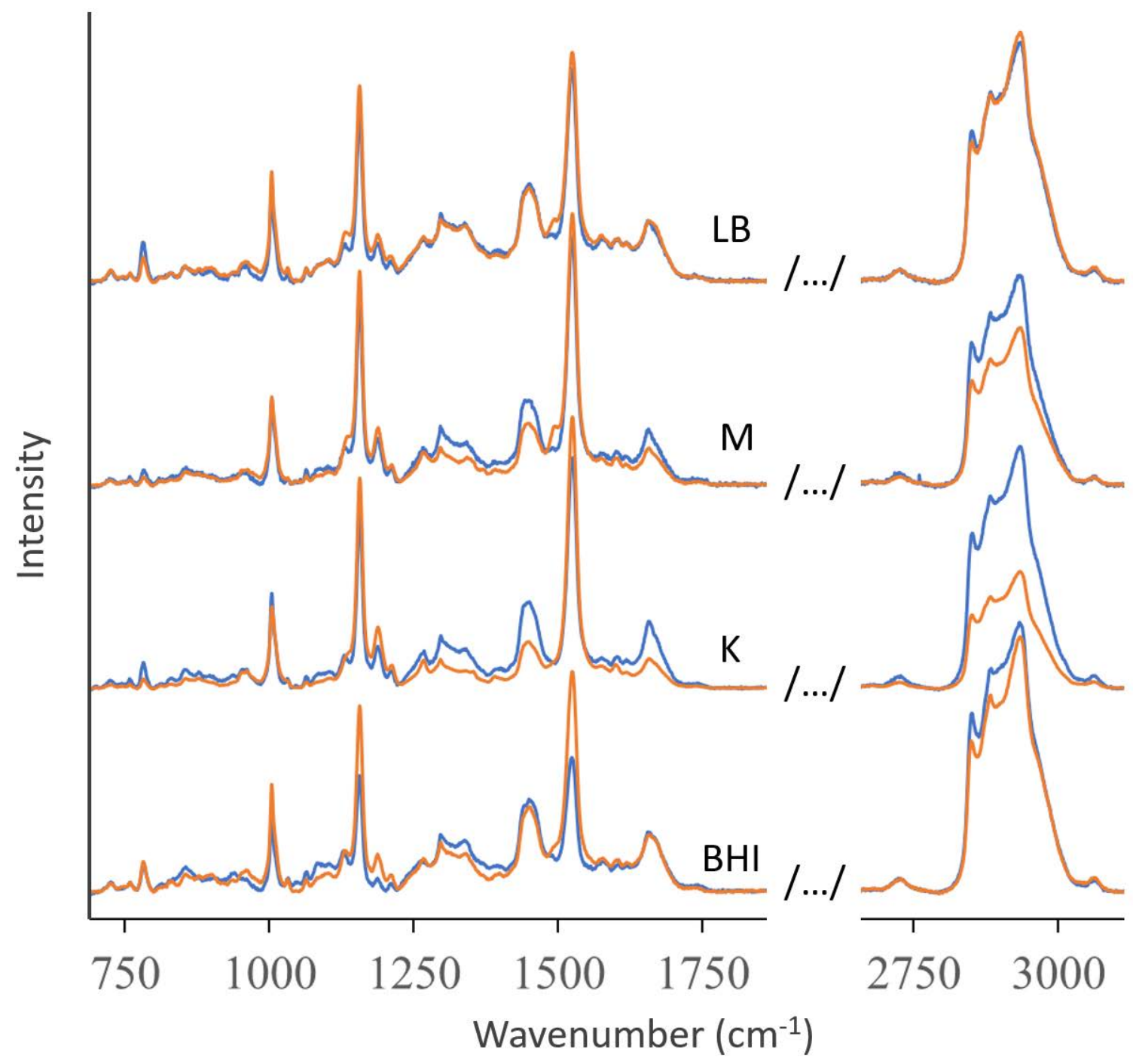

Figure 4.5. Raman spectra of Mycobacterium sp. MCS grown in Lysogeny Broth (LB), Middlebrook (M), Kircher (K), and Brain Heart Infusion (BHI) media for the log and stationary phases represented by blue and orange lines, respectively. 


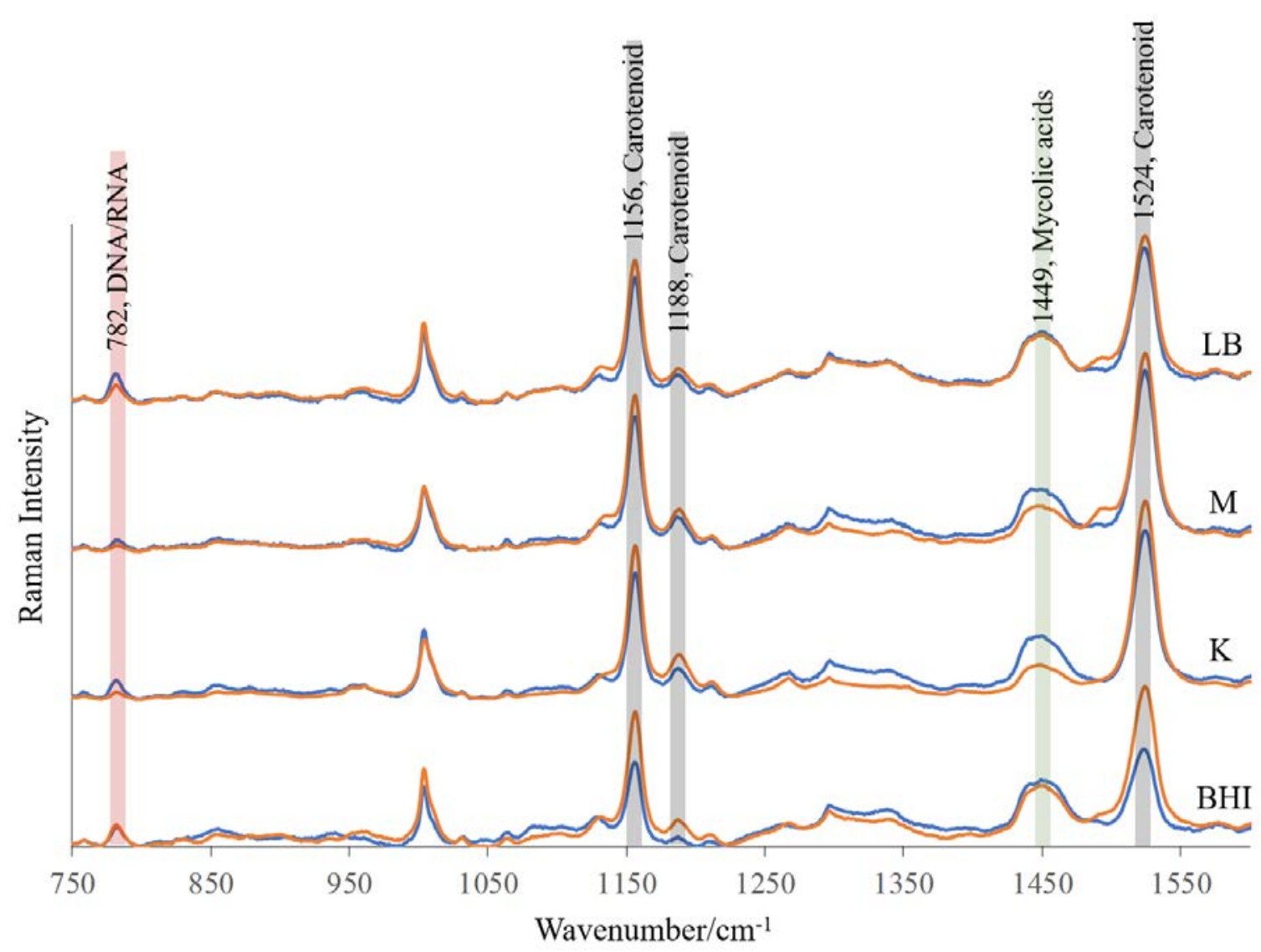

Figure 4.6. Raman spectra of Mycobacterium sp. MCS from $750-1600 \mathrm{~cm}^{-1}$ for Lysogeny broth LB, Middlebrook (M), Kircher (K), and Brain Heart Infusion (BHI) media for the log (blue) and stationary (orange) phases. Peaks highlighted in red, gray, and green mark peaks associated with DNA, carotenoids, and mycolic acids, respectively.

Stockel et al. [37] observed the changes in Raman spectra for $M$. aurum and $M$. smegmatis throughout the different growth phases. Although not explicitly stated in the article, the spectral figures indicate that Raman peaks for mycolic acids become dwarfed by carotenoid-related peaks as pigmented mycobacteria age. This trend was also found to be the case for Mycobacterium sp. MCS with the $1449 \mathrm{~cm}^{-1}$ mycolic acid peak and the 
carotenoid-related peaks (1156, 1188, and $1524 \mathrm{~cm}^{-1}$ ). The carotenoid peaks all had distinct increases while the mycolic acid peak decreased from log to stationary phases as seen in Figure 4.6. Overall, spectra of Mycobacterium sp. MCS followed the general trends found in literature of increasing carotenoids and decreasing DNA-related Raman intensities when transitioning from the log to the stationary phase.

\section{Conclusions}

In this study, colonies of Mycobacterium sp. MCS were grown in LB, Kirchner, Middlebrook, and Brain Heart Infusion broth. The influence of culture media and growth phase on Raman spectra of Mycobacterium sp. MCS was investigated. Using LDA, the $\log$ and stationary growth phases could be classified regardless of media with $94.8 \%$ accuracy. Grouping spectra according to growth phase, media could be classified at 85.4\% and 93.8\% accuracy for the log and stationary phases, respectively. LDA results indicate spectral differentiation according to media with greatest accuracy in the stationary phase. Trends among the log and stationary growth phases were also identified by spectral observation. Generally, carotenoid peaks increased while DNA/RNA-related peaks decreased as the bacteria aged from the log to the stationary phase, which is consistent with previous findings [36,37]. In addition, the peak assigned to mycolic acid decreased as the bacteria aged. These spectral changes are attributed to cell function and physiology associated with each phase.

Results indicate the need to account for spectral variances due to growth media and phase when building a spectral library for bacterial identification. The high classification accuracy (85.4\%-94.8\%) indicates detectable differences according to growth phase and growth media. As such, bacterial classification methods that 
incorporate Raman spectroscopy must account for these variations when developing a spectral library. Ideally, a Raman spectral library will include all growth phases and a wide range of culture media. Accounting for these variances will allow for greater accuracy in bacterial identification by Raman spectroscopy. 


\section{CHAPTER V}

\section{ALTERNATIVE CDEP DESIGN TO FACILITATE CELL ISOLATION FOR IDENTIFICATION BY RAMAN SPECTROSCOPY ${ }^{2}$}

\section{Abstract}

Dielectrophoresis (DEP) uses non-uniform electric fields to cause motion in particles due to the particles' intrinsic properties. As such, DEP is a well-suited label-free means for cell sorting. Of the various methods of implementing DEP, contactless dielectrophoresis (cDEP) is advantageous as it avoids common problems associated with DEP, such as electrode fouling and electrolysis. Unfortunately, cDEP devices can be difficult to fabricate, replicate, and reuse. In addition, the operating parameters are limited by the dielectric breakdown of polydimethylsiloxane (PDMS). This study presents an alternative way to fabricate a cDEP device allowing for higher operating voltages, improved replication, and the opportunity for analysis using Raman spectroscopy. In this device, channels were formed in fused silica rather than PDMS. The device successfully trapped $3.3 \mu \mathrm{m}$ polystyrene spheres for analysis by Raman spectroscopy. The successful implementation indicates the potential to use cDEP to isolate and identify biological samples on a single device.

\section{Introduction}

The use of label-free cell sorting, isolation, and identification techniques is becoming increasingly popular for analyzing biological samples. These techniques take advantage of cells’ intrinsic properties such as size, shape, or electrical polarizability to

\footnotetext{
${ }^{2}$ This paper was published in Sensors and coauthored by Elizabeth Vargis.
} 
perform the required analyses. One label-free method of identification is Raman spectroscopy, which correlates inelastic light scattering with specific vibrational and rotational modes of the target molecule or cell. One common method for cell sorting and isolation prior to acquiring Raman spectra is dielectrophoresis (DEP), which is the phenomenon where a non-uniform electric field causes motion of a particle. When using DEP, the manipulation of particles is based on the applied electric field and the particles' size, shape, and electrical properties.

There are several ways to implement DEP. A review of the mathematics of DEP and the various ways to implement DEP is beyond the scope of this article. However, several reviews and sources are available [9,11,13,14,131]. Briefly, in the early 1990s, DEP devices were made by embedding metallic electrodes within a sample chamber with a specific orientation or shape to create the non-uniform electric field. Unfortunately, these designs were prone to problems such as electrode fouling, electrolysis, Joule heating, and spatial limitations or how close the cells must be to the electrodes to be influenced by the electric field (approximately $30 \mu \mathrm{m}$ ). This limitation affects device efficiency and throughput. An alternative method to avoid common issues associated with DEP is insulator-based DEP (iDEP). In iDEP, electrodes are placed on opposite ends of a microfluidic device in direct contact with the sample solution. Insulating structures such as channel constrictions, sawtooth patterns, or an array of posts are placed within the channel between the electrodes. This arrangement forces the electric field to move around the structures, creating a non-uniform electric field required for DEP. Insulator-based DEP devices require high voltages to operate and are prone to 
electrolysis. Another DEP-based method that may address these drawbacks is contactless DEP (cDEP).

Contactless DEP creates a non-uniform electric field by insulating barriers within the sample channel as seen with iDEP. However, the electrodes in cDEP devices do not have physical contact with the sample channel. Instead, a thin insulating barrier separates liquid electrodes from the sample channel. This method is well-suited for biological samples as it minimizes the negative effects of electrolysis, electrode fouling, and Joule heating experienced by other common forms of DEP [11,132,133]. Fabricating a typical cDEP device involves polydimethylsiloxane (PDMS) casting on a silicon master mold (made previously using dry etching processes), removing the PDMS from the mold, and bonding the PDMS to glass [11]. The final structure requires a good seal of a thin PDMS membrane ( $20 \mu \mathrm{m}$ thick) to a glass substrate over $1-2 \mathrm{~cm}$ in length with typical channel depths of $50 \mu \mathrm{m}$. The device can be difficult to fabricate and replicate consistently as small defects during casting, de-molding, and bonding can occur, requiring many casts to produce one that will function properly.

Regardless of the way DEP is implemented, it is a powerful label-free tool to sort biological samples without tags, fluorescent markers, or specific DNA sequences for subsequent identification. It should be noted that DEP is not the only technique available for label-free means to sort and analyze cells. For example, laser tweezers Raman spectroscopy (LTRS) can trap, identify, and sort single cells [134-136]. Cells are targeted under a microscope and optically trapped using a laser. While trapped, a Raman spectrum is acquired to identify the cell. The cell can then be moved to another area based on the Raman spectra acquired. This technique is successful, but cannot meet the demands of 
high throughput as a Raman spectrum is acquired from each individual cell. In contrast, DEP can be used to automatically sort cells based on the cells' intrinsic properties allowing for higher throughput.

To meet the need for isolation and identification, DEP is coupled with other techniques such as Raman spectroscopy (DEP-Raman spectroscopy) to isolate and identify biological samples. For example, several researchers have used a quadruple electrode arrangement to concentrate bacteria by negative DEP for Raman analysis [9294]. Although successful, the design appears impractical as it is meant for small sample volumes $(\sim 200 \mu \mathrm{L})$ with some of the studies injecting even smaller volumes $(10 \mu \mathrm{L})$ of concentrated bacteria at the DEP site for successful demonstration [92,93]. Not only is the sample size problematic, but the design is prone to common DEP-related issues as stated previously and is ill-suited to analyze samples containing more than one bacteria at a time. Other examples of DEP-Raman spectroscopy include sample labeling using Raman reporters or antibodies [95,96]. The use of labels increases costs, limits shelf life, and may result in wasted materials due to the broad range of bacteria strains that can be present in a sample. Label-free identification methods are appealing to cut costs, increase simplicity, and reduce the risk of false positives. In previous cases where DEP-Raman spectroscopy systems did not use labels or tags, the device was made using traditional metallic electrodes in contact with the sample channel [61,97], exposing the device to standard DEP problems of electrode fouling and electrolysis.

This article proposes a new design to improve operating parameters, address fabrication issues associated with cDEP, and allow for simultaneous acquisition of Raman spectra without interference from PDMS. Thus, the design offers label-free 
sorting and identification of a sample at the same time. The design was tested with polystyrene spheres as a proof of concept. Results indicate successful application of cDEP to trap particles for acquiring the Raman spectra. To the authors' best knowledge, this is the first demonstration of using Raman spectroscopy and cDEP simultaneously.

\section{Materials and Methods}

\subsection{Device fabrication}

The microfluidic device was constructed in a layered structure as illustrated in Figure 5.1. Outer plates were made using Stratasys VeraClear photopolymer and Objet260 Connex3 printer (Eden Prairie, MN, USA). The outer plates accommodate \#832 screws to provide proper sealing of the device, as PDMS and fused silica do not bond easily.

The PDMS layer was made from Dow Corning 184 Sylgard (Auburn, MI, USA) silicone elastomer. A 10:1 ratio of PDMS monomer to curing agent was mixed, degassed, and poured onto a silicon wafer to provide a flat surface. The PDMS was cured at $100{ }^{\circ} \mathrm{C}$ for 35 min. After curing, the PDMS structure was carefully removed and trimmed. Holes were punched out using Miltex (Integra LifeSciences, York, PA, USA) $1.5 \mathrm{~mm}$ and $5 \mathrm{~mm}$ biopsy punches. The PDMS structure was aligned with the 3D printed plates and fastened to the fused silica microfluidic plate using \#8-32 screws. 


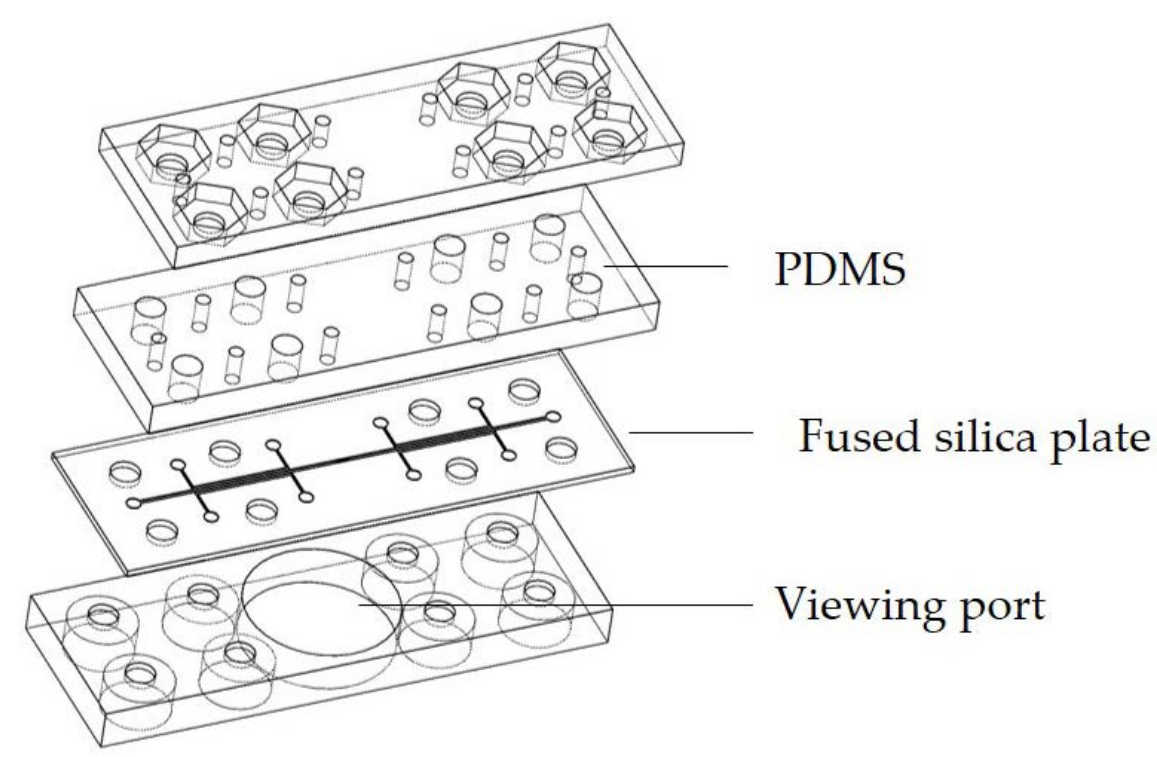

Figure 5.1. Illustration of the layered microfluidic device. Top and bottom plates were 3D printed with holes to accommodate \#8-32 screws. The bottom plate is equipped with a viewing port for an inverted microscope. The second layer from the top is made of PDMS. The second plate from the bottom is the fused silica microfluidic plate.

The fused silica microfluidic chip was fabricated by Translume (Ann Arbor, MI, USA). Figure 5.2a is an illustration of the microfluidic chip and Figure 5.2b is a microscope image of the sample channel with a square (100 $\mu \mathrm{m}$ by $100 \mu \mathrm{m})$ pillar array. The barriers between the liquid electrode and sample channels are $30 \mu \mathrm{m}$. The sample channel depth and width are $150 \mu \mathrm{m}$ and $500 \mu \mathrm{m}$, respectively, with $20 \mu \mathrm{m}$ set between each pillar in the array. 


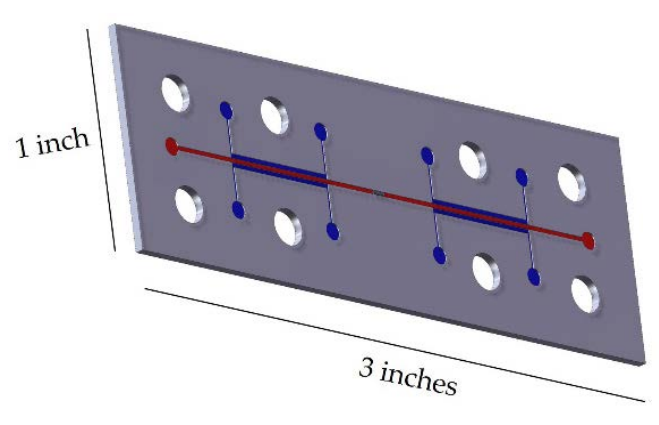

(a)

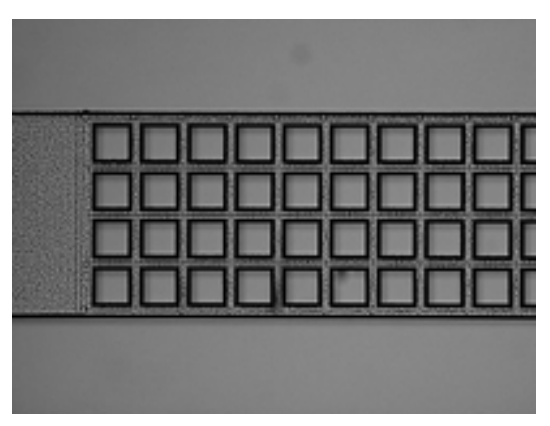

(b)

Figure 5.2. (a) Illustration of the fused silica microfluidic chip. Liquid electrodes and the sample channels are indicated by blue and red lines, respectively. The array of 4 by 15 square pillars act as insulating barriers in the middle of the sample channel. (b) Microscope image of the middle of the microfluidic sample channel showing the array of pillars in the middle of the sample channel. Pillar dimensions are $100 \mu \mathrm{m}$ by $100 \mu \mathrm{m}$.

\subsection{Sample preparation}

Polytetrafluoroethylene (PTFE \#20 AWG) tubing (Cole Parmer, Vernon Hills, IL, USA) was used to fill the sample and electrode channels. Two hundred microliter (Thermo Fisher Scientific, Waltham, MA, USA) pipette tips were trimmed to provide space for the microscope condenser and inserted through the PDMS to act as reservoirs for the liquid electrode channels. The sample consisted of 0.005× PBS (ScyTek, Logan, UT, USA), 0.1\% TWEEN 20 (ScyTek), and $3.3 \mu \mathrm{m}$ polystyrene fluorescent spheres (Thermo Fisher Scientific) at a concentration of approximately $2 \times 10^{7}$ particles per milliliter. The sample had a conductivity of $40 \mu \mathrm{S} / \mathrm{cm}$. Liquid electrodes were filled with $1 \times$ PBS with a conductivity of $15 \mathrm{mS} / \mathrm{cm}$. Dilute 3,3'-diethylthiatricarbocyanine iodide (DTTC) dye (Sigma-Aldrich, St. Louis, MO, USA) at a $250 \mu \mathrm{M}$ concentration in $1 \times$ PBS 
was added to the liquid electrodes to aid in visualizing during priming of the channels. The final concentration of DTTC in the channels was approximately $1 \mu \mathrm{M}$ after inserting the pipettes filled with $1 \times$ PBS. Copper wires (28 gauge) were used to connect the electrodes spanning over the sample channel as shown in Figure 5.3.

\subsection{Experimental setup}

A sinusoid wave was generated by an OWON AG1022 waveform generator (Industry, CA, USA). The signal was passed through a Trek Model 2205 high-voltage amplifier (Lockport, NY, USA) and monitored using an EZ Digital OS-5030 oscilloscope (Gyeonggi-do, Korea). The applied AC field (350 V $\mathrm{V}_{\mathrm{RMS}}$ at $100 \mathrm{~Hz}$ ) was delivered to the device using alligator clips. The sample flow was controlled by a New Era Pump Systems, Inc. NE-300 syringe pump (Farmingdale, NY, USA) operating at $5 \mu \mathrm{L} / \mathrm{h}$ during analysis.

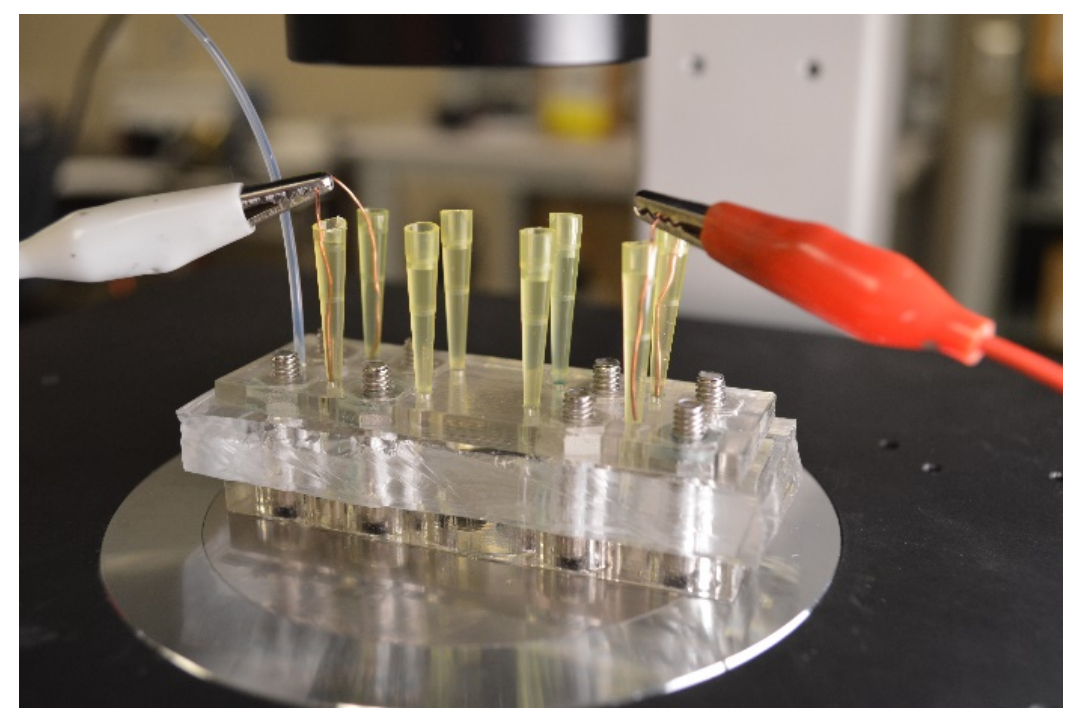

Figure 5.3. Image of the layered microfluidic device in operation set a-top an inverted microscope for analysis. 
Raman spectra were collected using an in-house built Raman microscope unit as described and used previously $[137,138]$. The unit consists of an inverted Nikon Eclipse TE2000-S (Melville, NY, USA), a 785 nm single-mode laser (Innovative Photonic Solutions, Monmouth, NJ, USA), an IsoPlane 160 spectrometer equipped with a 1200 g/mm grating (Princeton Instruments, Trenton, NJ, USA), and a Pixis-400 CCD (Princeton Instruments). A 25 s integration time was used to acquire one spectrum. Spectra were processed using LightField (Princeton Instruments) and Renishaw Wire 4.1 (Gloucestershire, United Kingdom).

\section{Results}

\subsection{Contactless dielectrophoresis}

The cDEP device was used to demonstrate trapping of a sample containing fluorescent polystyrene spheres $\left(\sim 2 \times 10^{7}\right.$ particles/mL, PSS). Figure 5.4 is a $20 \times$ magnification of the device under operation. The applied AC field consisted of $350 \mathrm{~V}_{\mathrm{RMS}}$ and $100 \mathrm{~Hz}$, while the flow rate through the device was $5 \mu \mathrm{L} / \mathrm{h}$. Particles were primarily trapped at the beginning of the pillar array. Trapping of the particles is necessary for subsequent evaluation using Raman spectroscopy. A video of the trapping process is included in the Supplementary Materials. 


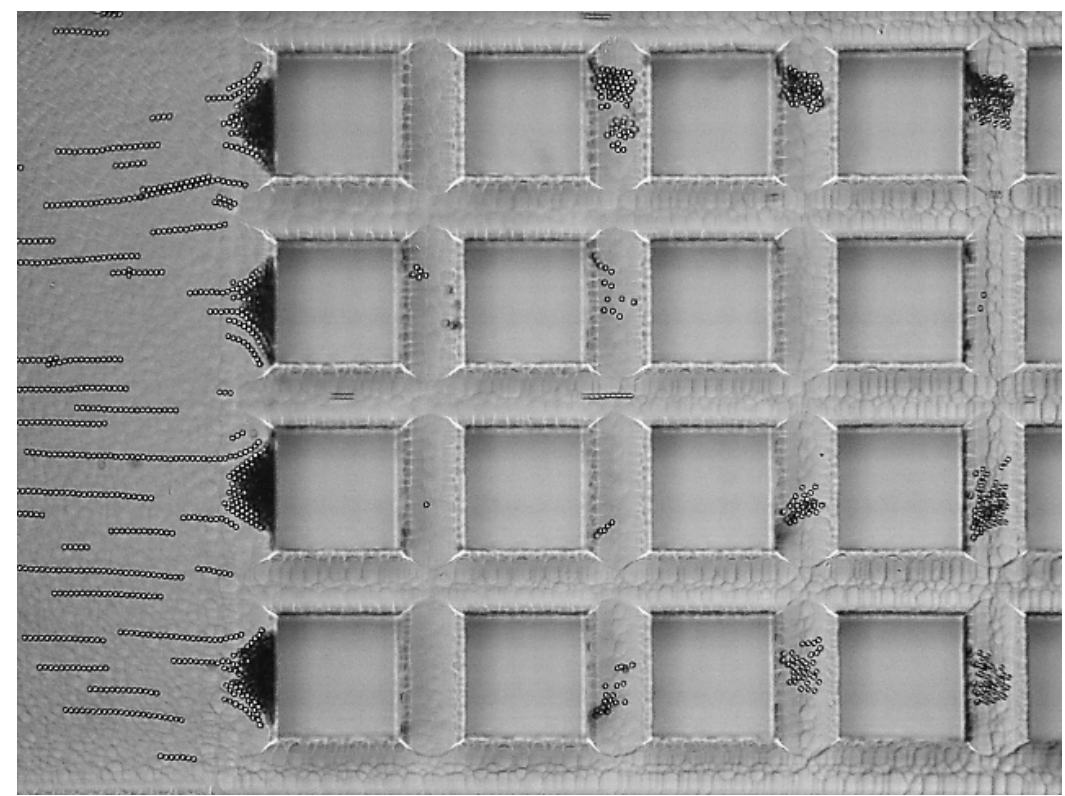

Figure 5.4. Image of cDEP device under operation (350 $\mathrm{V}_{\mathrm{RMS}}, 100 \mathrm{~Hz}, 5 \mu \mathrm{L} / \mathrm{h}$ ), trapping polystyrene spheres with a diameter of $3.3 \mu \mathrm{m}$. Square pillars are $100 \mu \mathrm{m}$ by $100 \mu \mathrm{m}$.

\subsection{Raman spectroscopy}

While the particles were trapped at the first set of pillars in the DEP device, a Raman spectrum was collected using a $785 \mathrm{~nm}$ wavelength laser at $15 \mathrm{~mW}$ for $25 \mathrm{~s}$ through a $40 \times$ objective lens. The resulting spectra were collected using LightField with a single 25-second acquisition and analyzed using Renishaw Wire 4.1. The spectrum of PSS trapped in the device was compared to positive and negative controls displayed in Figure 5.5 with a $y$-axis offset. From top to bottom, the spectra consist of PSS trapped under DEP, PSS on a quartz cover slip, PDMS, and the quartz coverslip with $0.005 \times$ PBS. 


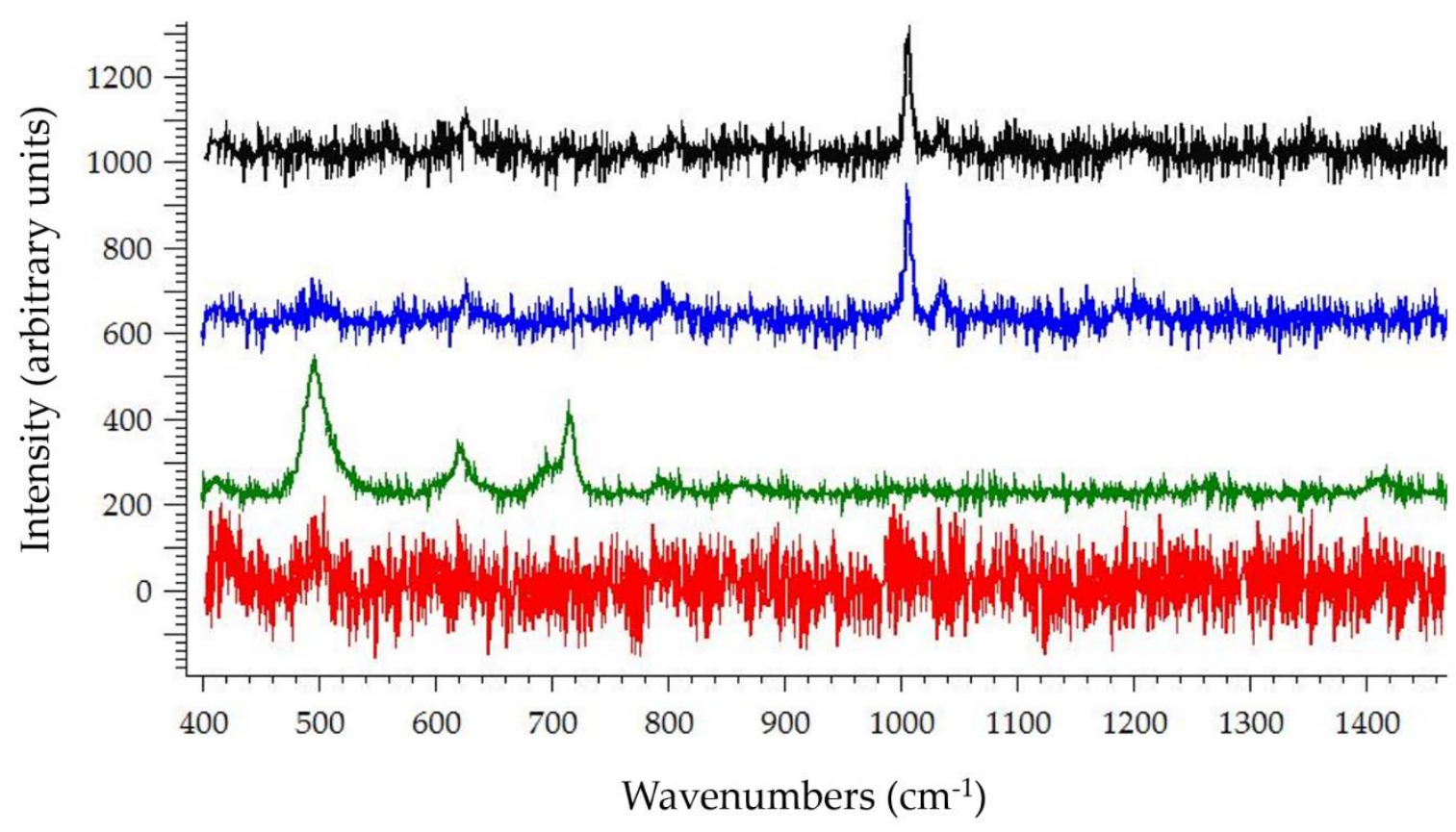

Figure 5.5. Raman spectra of $3.3 \mu \mathrm{m}$ PSS trapped within the cDEP device (black), PSS on quartz coverslip (blue), PDMS (green), and the quartz coverslip with $0.005 \times$ PBS (red). The spectrum of PSS on a quartz coverslip (blue) is the positive control. The spectra of PDMS (green) and the quartz coverslip without PSS (red) are negative controls.

\section{Discussion}

This study successfully demonstrates a unique form of implementing cDEP, which provides several advantages over traditional cDEP fabrication methods. The microfluidic channels of a traditional cDEP device are in the PDMS structure itself, where the barriers between the liquid electrode and sample are composed of PDMS. In addition, traditional cDEP devices use PDMS structures or channel wall constrictions to form the non-uniform electric fields. This study used fused silica to form the barriers and insulating structures, while PDMS was used to seal the device. This design provides a greater voltage operating range and enhanced reusability. 
Traditional cDEP devices are limited according to the dielectric breakdown of PDMS. Literature provides a wide range of dielectric breakdown values from 129 to 635 $\mathrm{V} / \mu \mathrm{m}$ for thin membranes $(2-20 \mu \mathrm{m})$ depending on PDMS thickness and electrode shape $[139,140]$. Yet, research articles concerning cDEP experiments report much lower dielectric breakdown values such as $20 \mathrm{~V} / \mu \mathrm{m}$ [141] or $14 \mathrm{~V} / \mu \mathrm{m}$ [142]. Table 5.1 lists publications that implemented cDEP for cell manipulations and includes the associated voltages, frequencies, and flow rates used. Most cDEP research articles do not use more than 250 V. To the authors' knowledge, only one other research article has reported using $350 \mathrm{~V}$ during operation [143]. In [143], the device had a channel depth of $50 \mu \mathrm{m}$ with microfluidic structures composed of PDMS at a 10:1 ratio. The barrier between the liquid electrodes and sample channel was made of PDMS with a 5:1 ratio $13 \mu \mathrm{m}$ thick. The device was designed to prevent pearl chain formation, where particles are attracted to each other due to dipole-dipole interactions and are affected by particle size and concentration. They found that reducing pillars to sizes similar to target cells improved trapping efficiency and reduced pearl chaining. While the device from Čemažar et al. [143] has a high trapping efficiency and selectivity, it is only meant for isolation and enrichment before further off-chip analysis. The cDEP device presented here adds the advantage of on-chip isolation, enrichment, and analysis. 
Table 5.1. List of cDEP publications and their associated operating parameters. As the list consists of alternating current sources, voltage is expressed in root mean square $\left(\mathrm{V}_{\mathrm{RMS}}\right)$.

\begin{tabular}{cccc}
\hline Source & Voltage (VRMs) & Frequency (kHz) & Flow Rate $(\boldsymbol{\mu L} / \mathbf{h})$ \\
\hline$[144]$ & 200 & $5-50$ & 5 \\
{$[145]$} & 200 & $5-70$ & 5 \\
{$[146]$} & 250 & 500 & Not reported $^{1}$ \\
{$[141]$} & 250 & 600 & 1000 \\
{$[147]$} & $227-250$ & $50-100$ & 10 \\
{$[148]$} & $20-50$ & $120-320$ & 20 \\
{$[142]$} & $70-190$ & 300 & 20 \\
{$[149]$} & $20-150$ & $140-500$ & 20 \\
{$[132]$} & 250 & 85 & $10-15$ \\
{$[150]$} & $200-300$ & $10-70$ & 5 \\
{$[151]$} & $50-200$ & $200-600$ & 20 \\
{$[143]$} & $250-350$ & 30 & $1200-2160$ \\
Current article & 350 & 0.1 & 5 \\
\hline
\end{tabular}

${ }^{1}$ Rate driven by electrokinetic flow.

The applied voltage used in this paper was limited by the available equipment. With the aid of a step-up transformer or other equipment modifications as suggested in [152], higher voltages can be obtained without approaching the dielectric breakdown of fused silica (950 V/ $\mu \mathrm{m}$ [153]) while maintaining a range of optimal and commonly used frequencies (1-1000 kHz [147]). Future work will make use of such equipment to demonstrate how fused silica can provide a higher range of applied voltage due to the dielectric breakdown. In addition to improved voltage range, the use of fused silica allows for acquisition of Raman spectra without interference of a PDMS signature as demonstrated in Figure 5.5. The device can be reused and therefore provide more reliable results.

It should be noted that the relationship between voltage and frequency requirements varies with cDEP device. Sano et al. [147] demonstrated that, for cDEP devices, voltage drop and associated electric field gradients can vary according to 
geometric configuration and applied frequency. Low frequencies cause a smaller percentage of the voltage drop to occur across the sample channel and therefore generate smaller DEP force vectors. In contrast, the use of higher frequencies causes a higher percentage of the voltage drop to occur within the sample channel, therefore lowering the voltage demand. Our study used a very low frequency $(100 \mathrm{~Hz})$ to demonstrate separation of particles due to negative DEP (particles drawn to areas with a low electric field gradient). Although the frequency used in this study was considerably lower than what is used for typical separations, it demonstrated that DEP separation can be achieved even under unfavorable conditions.

Future work will address issues raised from the current design. For example, the flow rate used for this study was the same or slower than other research articles, as listed in Table 5.1. To be competitive at providing rapid analyses, a faster flow rate will need to be achieved. Device features such as pillar size, shape, and spacing will also be changed to accommodate $1-\mu \mathrm{m}$-sized particles, as the end goal is to trap bacteria and prevent pearl chain formation. The current setup primarily traps particles at the first column of pillars with secondary trapping within the array likely due to pearl chain formations. As the design was created as a proof of concept to perform cDEP and Raman spectroscopy simultaneously, the authors acknowledge that the arrangement has not been optimized for trapping efficiency. To improve trapping efficiency and selectivity, smaller pillar sizes will be incorporated with columns of pillars spaced further away from each other in future devices. In addition, structures will need to be arranged for simultaneous separation of multiple particles in a sample as demonstrated in other studies [77,154]. 


\section{Conclusions}

A cDEP device was successfully fabricated which demonstrated simultaneous trapping and Raman analysis of $3.3 \mu$ m polystyrene spheres. The device is constructed with microfluidic channels etched into fused silica, allowing for a greater voltage operating range and improved reusability compared to typical cDEP designs. To the authors' knowledge, this article presents the first demonstration where Raman spectroscopy was performed on a cDEP device. 


\section{CHAPTER VI}

\section{SEPARATION OF A MIXED SAMPLE USING CDEP}

\section{Abstract}

Bacterial cell isolation and identification are two major factors contributing to the high analysis time of bacteria. One way to decrease analysis times is by using dielectrophoresis (DEP), a common technique used for cell sorting and isolation, in conjunction with Raman spectroscopy for cell identification. DEP-Raman devices have been used for bacterial analysis; however these devices have a number of drawbacks whether it be sample heating, cell-to-electrode proximity, electrode fouling, or inability to address sample debris. We propose a cDEP-Raman device to simultaneously isolate and identify particles from a mixed sample. The device successfully separated a mixed sample of bacteria and $5 \mu \mathrm{m}$ polystyrene spheres, thereby providing a platform to decrease the analysis time of bacteria.

\section{Introduction}

Dielectrophoresis (DEP) is a technique that is often used for cell sorting, isolating, and trapping. It involves the manipulation of particles in a non-uniform electric field based on the physical and electrical properties of the particle. DEP has been used for sorting of many biological samples such as bacteria [68,83,84], DNA [155-157], and red blood cells [158-160]. Although DEP is effective for cell isolation, it is often coupled with other techniques to provide quantitative and qualitative information. For example, DEP has been integrated with impedance analysis (DEPIA) for bacterial concentration and quantification [88-91]. The device can also be equipped with immunoglobulins for 
detection and identification as a bacteria binds to an associated immunoglobulin and results in a change in impendence [89]. This identification scheme is limited to antibiotic labels and is prone to false positives due to non-specific binding. In addition, the methods require the use of metallic electrodes, which exposes the technique to common problems associated with DEP such as electrode fouling, electrolysis, and Joule heating.

Coupling DEP with other techniques such as Raman spectroscopy (DEP-Raman spectroscopy) has been useful for concentrating and then identifying bacteria, but it has limitations due to design, scalability, or labeling. For example, several researchers have used a quadruple electrode arrangement to concentrate bacteria by negative DEP for Raman analysis [92-94]. Although successful, the design appears limited as it is meant for small sample volumes ( $200 \mu \mathrm{L}$ ), and some of the studies used only $10 \mu \mathrm{L}$ of concentrated bacteria at the DEP site for successful demonstration [92,93]. Not only is the sample volume problematic, but the design is prone to common DEP-related issues as stated previously and is not suited to analyze mixed samples. Some DEP-Raman spectroscopy devices include the use of labels in the form of Raman reporters and/or antibiotics [95,96]. The use of labels increases costs, limits shelf life, and may result in wasted materials due to the broad range of bacteria strains that can be present in a sample. Label-free identification methods are appealing to cut costs, increase simplicity, and reduce the risk of false positives. In cases where DEP-Raman spectroscopy systems did not use labels or tags, the device was made using the traditional metallic electrodes in contact with the sample channel [61,97], exposing the device to typical DEP problems of electrode fouling and electrolysis. 
Construction of DEP devices has changed to eliminate or reduce problems associated with the DEP method. Early DEP devices used planar metallic electrodes and were prone to electrode fouling, such as bubble formation on the surface of the electrode. In addition, dielectrophoretic forces drop off quickly with increased distance from the electrode. These issues were addressed by the development of insulator-based DEP (iDEP). iDEP devices have electrodes on either end of the device with insulating structures at the center, which force non-uniformities in the electric field spanning from the bottom to the ceiling of the sample channel. Thus, the dielectrophoretic force is not dependent on proximity to electrodes, eliminating electrode fouling. Although electrolysis still occurs in iDEP devices, it does not occur at the site of DEP sorting or trapping. Contactless DEP (cDEP) is yet another form of DEP that eliminates common issues with DEP by the use of capacitive coupling. Like iDEP, cDEP incorporates insulator barriers at the DEP-active site to create the non-uniform electric field. cDEP differs from iDEP as electrodes are isolated from the sample channel by an insulated barrier, eliminating issues of electrode fouling and electrolysis. As such, cDEP is a great candidate to analyze biological samples as it is a gentler technique compared to other DEP forms.

In this article, we demonstrate the simultaneous isolation of bacteria from $5 \mu \mathrm{m}$ polystyrene spheres and the acquisition of Raman spectra using an adaptation of a cDEPRaman design previously introduced [2]. The successful isolation of bacteria from debris and simultaneous acquisition of Raman spectra indicate the potential for the device to decrease the analysis time of bacteria.

\section{Materials and Methods}




\subsection{DEP modeling}

The dielectrophoretic force acting on a spherical, homogenous particle is expressed as

$$
\vec{F}_{D E P}=2 \pi r^{3} \varepsilon_{m} \operatorname{Re}\left[f_{C M}\right] \nabla\left(\vec{E}_{r m s}^{2}\right)
$$

where r, $\nabla\left(\vec{E}_{r m s}^{2}\right)$, and $\varepsilon_{\mathrm{m}}$ are the sphere radius, gradient of the squared electric field, and electrical permittivity of the media, respectively. The subscript $r m s$ stands for root mean squared and is applicable for AC power sources. $R e\left[f_{C M}\right]$ is the real part of the ClausiusMossotti factor, which is expressed as

$$
\left[f_{C M}\right]=\frac{\varepsilon_{p}^{*}-\varepsilon_{m}^{*}}{\varepsilon_{p}^{*}+2 \varepsilon_{m}^{*}}
$$

where $\varepsilon_{p}^{*}$ and $\varepsilon_{m}^{*}$ is the complex permittivity of the particle and media, respectively. The complex permittivity is dependent on the conductivity $(\sigma)$ and the frequency of the applied field $(f)$ and is given by

$$
\varepsilon^{*}=\varepsilon-\frac{j \sigma}{2 \pi f}
$$

where $j$ is the square root of negative one.

To trap a particle, the dielectrophoretic force must be equal to the drag force acting on the particle. For laminar flow acting on a sphere, the drag force is expressed as

$$
\vec{F}_{D R A G}=6 \pi \eta r U
$$

where $\eta$ is the kinematic viscosity and $U$ is the velocity of the object relative to the fluid. Velocity is determined by dividing the flow rate by the cross sectional area of the sample channel at the pillar array. During trapping, the object is stationary relative to the fluid. Therefore, setting Equations (6.1) and (6.4) equal to each other and rearranging values results in 


$$
\nabla\left(\vec{E}_{r m s}^{2}\right)=\frac{3 \eta U}{r^{2} \varepsilon_{m} \operatorname{Re}\left[f_{C M}\right]} .
$$

Equation (6.5) is the required gradient of the squared electric field to trap a particle. It should be noted that in the case of a prolate ellipsoid, which is the case for mycobacteria, Equation (6.1) changes to

$$
\vec{F}_{D E P}=\frac{2 \pi a b^{2}}{3} \varepsilon_{m} R e\left[f_{C M}\right] \nabla\left(\vec{E}_{r m s}^{2}\right)
$$

where $a$ and $b$ are the major and minor axis of a prolate ellipsoid. The calculation of the Clausius-Mossotti factor is expressed as

$$
\left[f_{C M}\right]=\frac{\varepsilon_{p}^{*}-\varepsilon_{m}^{*}}{1+\left(\frac{\varepsilon_{p}^{*}-\varepsilon_{m}^{*}}{\varepsilon_{m}^{*}}\right) A} .
$$

Here, $A$ is the depolarization factor and is dependent on the respective $\mathrm{x}, \mathrm{y}$, or $\mathrm{z}$ axis. However, in the case of a prolate ellipsoid, the depolarization can be expressed by the expansion

$$
A=\frac{1}{3 \gamma^{-2}}\left[1+\frac{3}{5}\left(1-\gamma^{-2}\right)+\frac{3}{7}\left(1-\gamma^{-2}\right)^{2}+\cdots\right]
$$

where $\gamma$ is the major axis ( $a$ ) of the prolate ellipsoid divided by the minor axis $(b)$.

In the case of mycobacteria, a core-shell model can be used to determine the effective electrical permittivity. The core is the cytoplasm $\left(a_{4}, b_{4}, \varepsilon_{5}, \sigma_{5}\right)$, the cytoplasmic membrane is the first shell $\left(a_{3}, b_{3}, \varepsilon_{4}, \sigma_{4}\right)$, the cell wall is the second shell $\left(a_{2}, b_{2}, \varepsilon_{3}, \sigma_{3}\right)$, and the lipid layer is the third shell $\left(a_{1}, b_{1}, \varepsilon_{2}, \sigma_{2}\right)$. For core-shell configurations, the overall effective electrical permittivity must be determined and used in place of $\varepsilon_{p}^{*}$ from Equation (6.7) to calculate $\left[f_{C M}\right]$. The effective electrical permittivity is expressed as

$$
\varepsilon_{(i) e f f}^{*}=\varepsilon_{i}^{*}\left[\frac{\varepsilon_{i}^{*}+\left(\varepsilon_{(i+1) e f f}^{*}-\varepsilon_{i}^{*}\right)\left(A_{i}+v_{i}\left(1-A_{(i-1)}\right)\right)}{\varepsilon_{i}^{*}+\left(\varepsilon_{(i+1) e f f}^{*}-\varepsilon_{i}^{*}\right)\left(A_{i}+v_{i} A_{(i-1)}\right)}\right]
$$

with 


$$
v_{i}=\frac{a_{i} b_{i}^{2}}{a_{i-1}^{3}} .
$$

to calculate the required $\nabla\left(\vec{E}_{r m s}^{2}\right)$ to trap mycobacteria, the dielectric force expressed in Equation (6.6) must be set equal to the drag force of a prolate ellipsoid. According to Chwang and $\mathrm{Wu}$ [161], the drag force on a prolate ellipsoid with a very small Reynolds number is expressed as

$$
\vec{F}_{D R A G}=16 \pi \eta a U e \beta
$$

where $e$ is the eccentricity of the ellipsoid and

$$
\beta=2 e^{2}\left[2 e+\left(3 e^{2}-1\right) \log \left(\frac{1+e}{1-e}\right)\right]^{-1} .
$$

Setting Equation (6.6) and (6.11) equal to each other and rearranging results in the required gradient of the electric field squared as expressed in Equation (6.13).

$$
\nabla\left(\vec{E}_{r m s}^{2}\right)=\frac{24 \eta U e \beta}{b^{2} \varepsilon_{m} \operatorname{Re}\left[f_{C M}\right]}
$$

Table 6.1 provides values for variables used to determine the required $\nabla\left(\vec{E}_{r m s}^{2}\right)$ for polystyrene spheres (PSS) and mycobacteria using Equations (6.5) and (6.13). The effective electrical permittivity for bacteria were calculated using Equation (6.9). Values for electrical conductivity, electrical permittivity, and thickness of mycobacteria cytoplasm, cytoplasmic membrane, cell wall, and lipid layer for Mycobacterium sp. MCS was assumed to be the same as for M. smegmatis [162]. Overall shape and size of $M$. sp. MCS was calculated from AFM and SEM images from previous work at Utah State University [50]. Using the stated electrical and physical values and assuming a particle velocity of $23.1 \mu \mathrm{m} / \mathrm{s}$ resulted in $\nabla\left(\vec{E}_{r m s}^{2}\right)$ values of $9.69 \times 10^{14}$ and $3.13 \times 10^{13} \mathrm{~kg}^{2} \mathrm{~m} / \mathrm{s}^{6} \mathrm{~A}^{2}$ for bacteria and PSS, respectively. Figure 6.1 is a COMSOL-generated plot of expected $\nabla\left(\vec{E}_{r m s}^{2}\right)$ for the two pillar arrays operating at $500 \mathrm{~V}$ and $40 \mathrm{kHz}$. The modeling results 
indicate that the device is capable of isolating mycobacteria from $5 \mu \mathrm{m}$ polystyrene spheres.

Table 6.1. List of electrical permittivities, electrical conductivities, frequency, and the Clausius-Mossotti factor used to determine the required electric field gradient to induce trapping of bacteria and polystyrene spheres.

\begin{tabular}{ccccccc}
\hline$\varepsilon_{m}$ & $\varepsilon_{P S S}$ & $\sigma_{m}(\mathrm{~S} / \mathrm{m})$ & $\sigma_{P S S}(\mathrm{~S} / \mathrm{m})$ & $f(\mathrm{kHz})$ & $\operatorname{Re}\left[f_{\mathrm{CM}}\right]_{\mathrm{PSS}}$ & $\operatorname{Re}\left[f_{\mathrm{CM}}\right]_{\mathrm{MCS}}$ \\
\hline 80 & 2.5 & 0.01 & $1.0 \times 10^{-12}$ & 40 & -0.5 & -0.451 \\
\hline
\end{tabular}

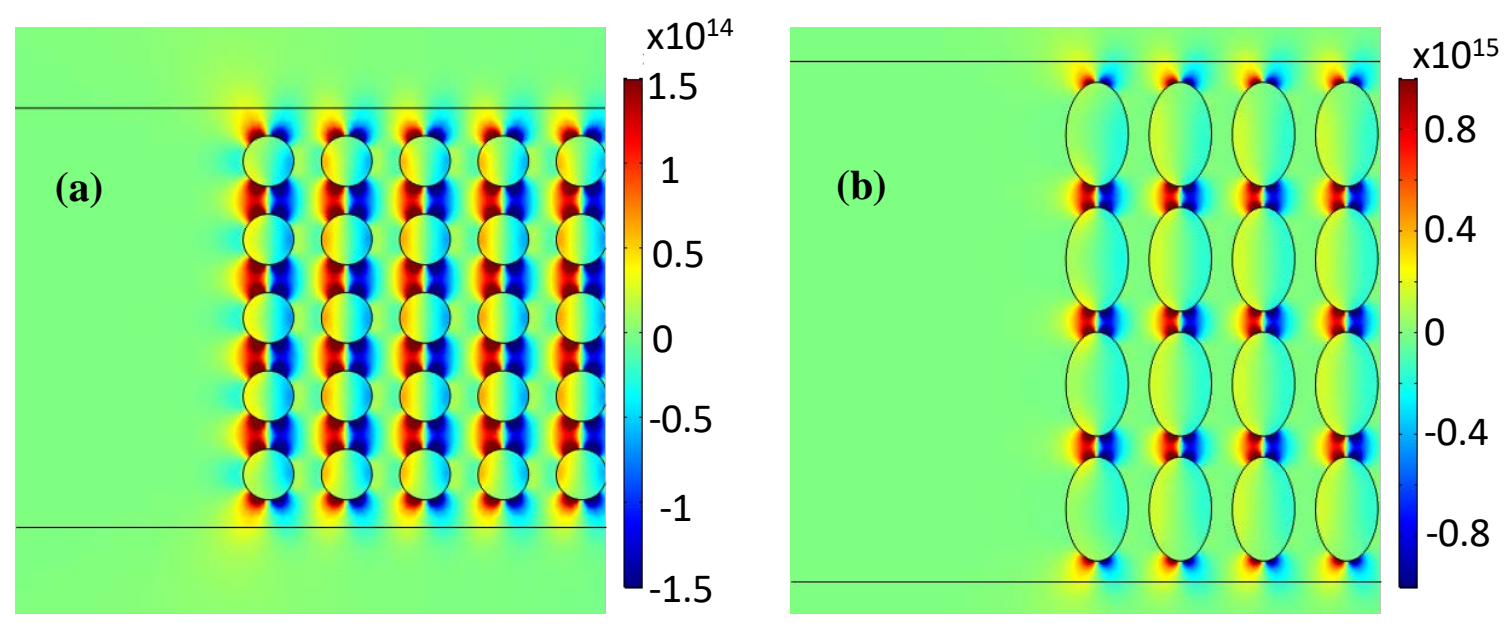

Figure 6.1. COMSOL simulations to determine expected gradient of the squared electric field for first (a) and second (b) pillar array operated at 500V and $40 \mathrm{kHz}$. Units for values are in $\mathrm{kg}^{2} \mathrm{~m} / \mathrm{s}^{6} \mathrm{~A}^{2}$. 


\subsection{Device fabrication}

Figure 6.2 illustrates the layered microfluidic device with outer plates, which were made using Stratasys VeraClear photopolymer and Objet260 Connex3 printer (Eden Prairie, MN, USA). The outer plates accommodate \#8-32 screws to provide proper sealing of the device, as bonding between PDMS and fused silica is avoided so the device can be cleaned and reused. The PDMS layer was made from Dow Corning 184 Sylgard (Auburn, MI, USA) silicone elastomer with a 10:1 ratio of PDMS monomer to curing agent. The solution was mixed, degassed, and poured onto a silicon wafer to provide a flat surface. The PDMS was cured at $100^{\circ} \mathrm{C}$ for 35 min. After curing, the PDMS structure was carefully removed and trimmed. Holes were punched out using Miltex (Integra LifeSciences, York, PA, USA) 1.5 mm biopsy punches. The PDMS structure was aligned with the 3D printed plates and fused silica plate. The device was held together using \#8-32 screws.

The fused silica plate was fabricated by Translume (Ann Arbor, MI, USA). Figure 6.3a is an illustration of the microfluidic chip, while Figures 6.3b and 6.3c are brightfield microscope images of the first and second pillar array, respectively. The barriers between the liquid electrode and sample channels are approximately $30 \mu \mathrm{m}$. The sample channel depth and width are $180 \mu \mathrm{m}$ and $500 \mu \mathrm{m}$, respectively. 


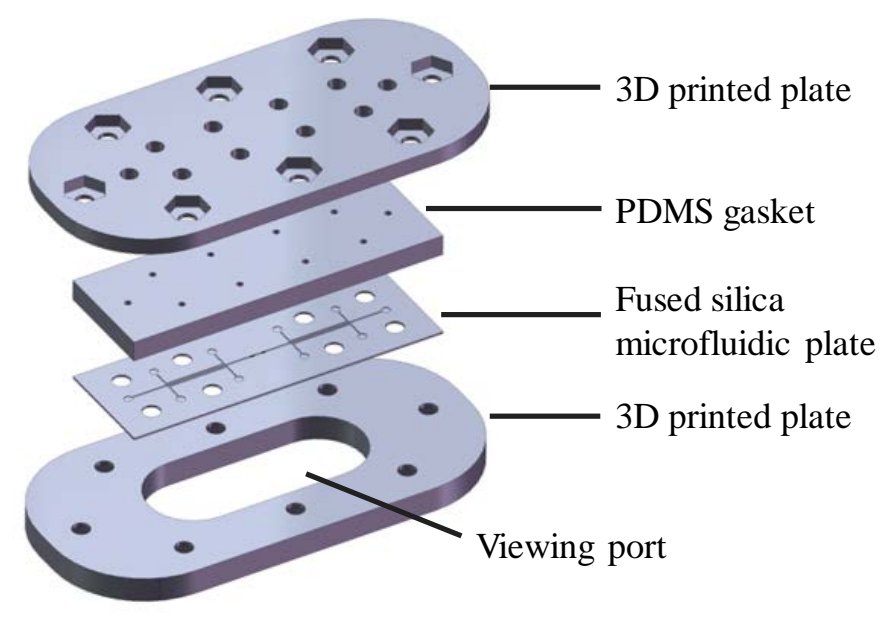

Figure 6.2. Schematic of layered cDEP device.
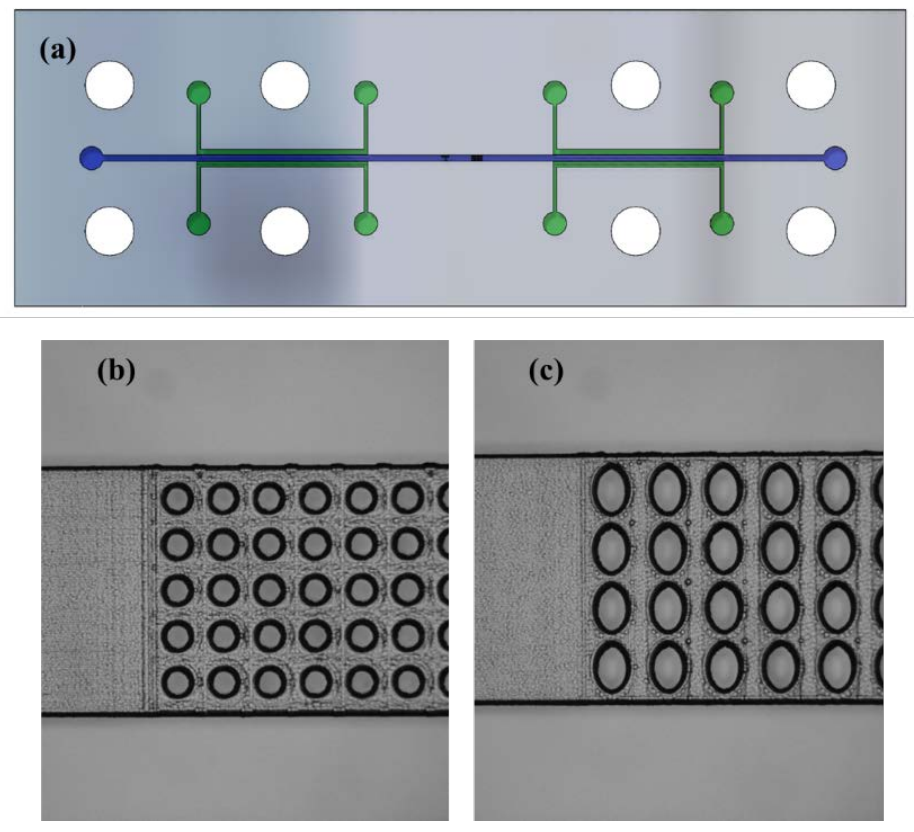

Figure 6.3. Schematic of fused silica microfluidic plate (a) with green and blue channels, indicating the liquid electrode and sample channels, respectively. Brightfield images of first (b) and second (c) pillar arrays positioned in the middle of the sample channel. In (b), pillars are $60 \mu \mathrm{m}$ diameter while in (c), the length of and width of the oval pillars are 100 and $60 \mu \mathrm{m}$, respectively. 


\subsection{Sample preparation}

The sample consisted of $0.005 \times$ PBS (ScyTek, Logan, UT, USA), 0.1\% TWEEN 20 (ScyTek), $5 \mu \mathrm{m}$ polystyrene fluorescent spheres (Thermo Fisher Scientific) at approximately $0.08 \%$ solids, and Mycobacterium sp. MCS. The bacteria were grown in Lysogeny Broth and introduced to the sample after rinsing. The rinsing process consisted of placing $1 \mathrm{~mL}$ of inoculated broth into a $1.5 \mathrm{~mL}$ centrifuge tube. The solution was centrifuged for 2min at 6000 rpm using a Thermo Fisher Scientific Heraeus Pico 21 microcentrifuge. The supernatant was then discarded and the bacteria pellet formed at the bottom was resuspended in a sterile $0.85 \%$ saline solution and vortexed to ensure proper mixing. This centrifuging process was performed three times before introduction to the sample solution with the polystyrene spheres.

The sample had a conductivity of $115 \mu \mathrm{S} / \mathrm{cm}$. Two hundred microliter (Thermo Fisher Scientific, Waltham, MA, USA) pipette tips were trimmed to provide space for the microscope condenser and inserted through the PDMS to act as reservoirs for the liquid electrode channels. Using polytetrafluoroethylene (PTFE \#20 AWG) tubing (Cole Parmer, Vernon Hills, IL, USA), liquid electrodes were filled with $1 \times$ PBS with a conductivity of $17 \mathrm{mS} / \mathrm{cm}$. Copper wires (28 gauge) were used to connect the electrodes spanning over the sample channel as performed previously [2].

\subsection{Experimental setup}

A sinusoid wave was generated by an OWON AG1022 waveform generator (Industry, CA, USA) and passed through a Trek Model 2205 high-voltage amplifier (Lockport, NY, USA). The signal was monitored using an EZ Digital OS-5030 oscilloscope (Gyeonggi-do, Korea). The applied AC field (500 V VMs at $33 \mathrm{kHz}$ ) was 
delivered to the device using alligator clips. The base sample $(0.005 \times$ PBS, $0.1 \%$ TWEEN 20) was introduced to the sample channel by means of capillary action. Once filled, the sample with the polystyrene spheres was introduced, and flow was generated by gravity due to height differences between the inlet and the outlet reservoirs.

Raman spectra of trapped bacteria were collected using an in-house-built Raman microscope unit as described and used previously $[137,138]$. The unit consists of an inverted Nikon Eclipse TE2000-S (Melville, NY, USA), a 785 nm single-mode laser (Innovative Photonic Solutions, Monmouth, NJ, USA), an IsoPlane 160 spectrometer equipped with a $1200 \mathrm{~g} / \mathrm{mm}$ grating (Princeton Instruments, Trenton, NJ, USA), and a Pixis-400 CCD (Princeton Instruments). A 60-s integration time was used to acquire one spectrum. Spectra were processed using LightField (Princeton Instruments) and Renishaw Wire 4.1 (Gloucestershire, United Kingdom).

\section{Results and Discussion}

Figure 6.4 displays brightfield of the cDEP device under operation to isolate bacteria away from debris. The $5 \mu \mathrm{m}$ polystyrene spheres were all trapped at the first pillar array (Figure 6.4a), while the bacteria were trapped at the first and second pillar array. Although some of the bacteria were trapped at the first array, the device was successful in isolating only bacteria at the second array, as seen in Figure 6.4b. Raman spectra of the bacteria were collected while the bacteria were trapped in the device. The Raman spectrum of Mycobacterium sp. MCS trapped in the CDEP device is displayed in Figure 6.5. This successful isolation of a mixed sample and identification by Raman spectra indicates the potential for use of this cDEP-Raman device to simultaneously isolate and identify bacteria. 

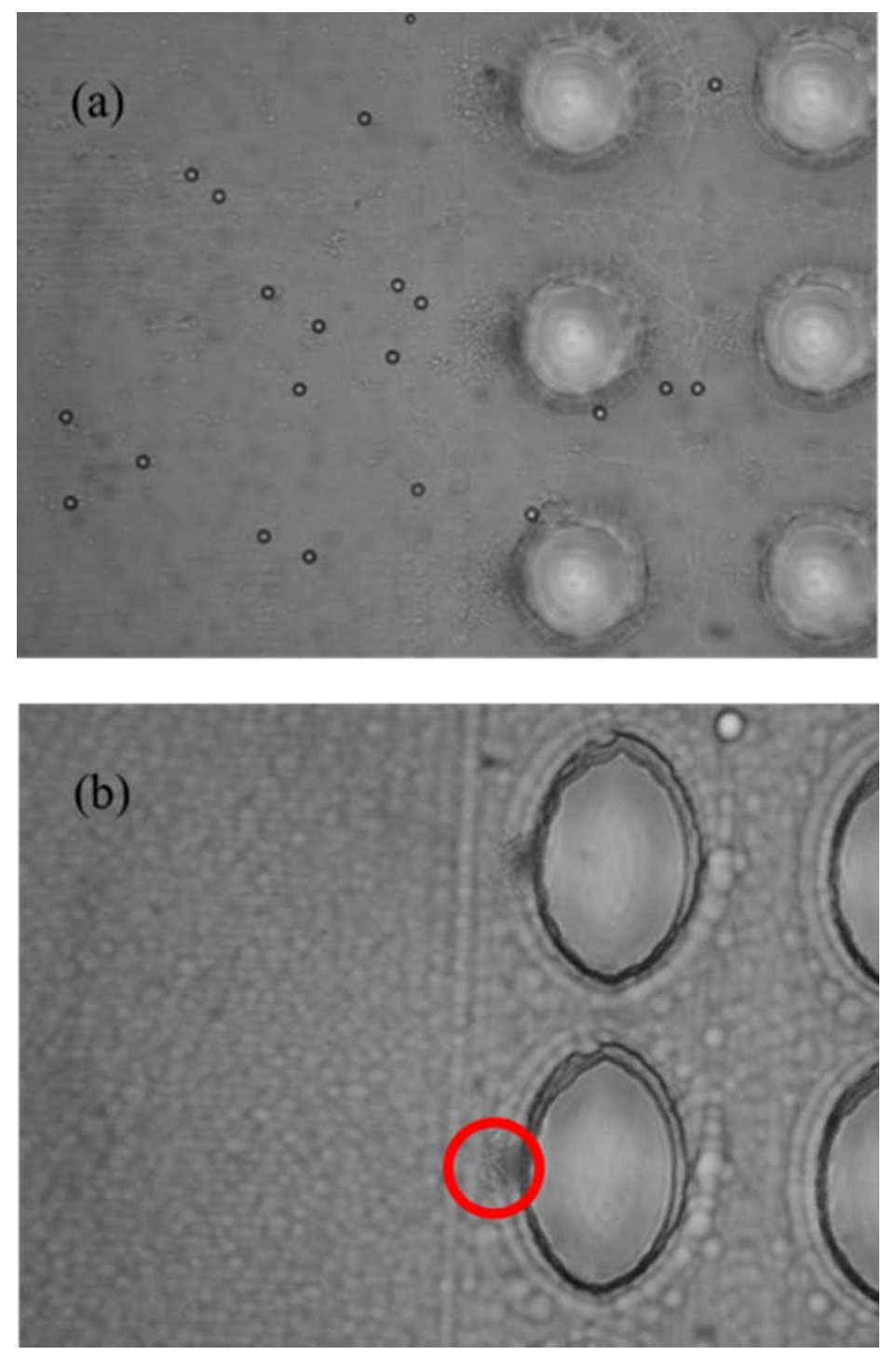

Figure 6.4. Brightfield images of isolating bacteria away from polystyrene spheres acting as debris. Image (a) is of the first pillar array with pillar diameters for $60 \mu \mathrm{m}$, while image (b) is of the second pillar array with the length of the oval pillar being $100 \mu \mathrm{m}$. The red circle indicates the area where Raman spectra were collected. 


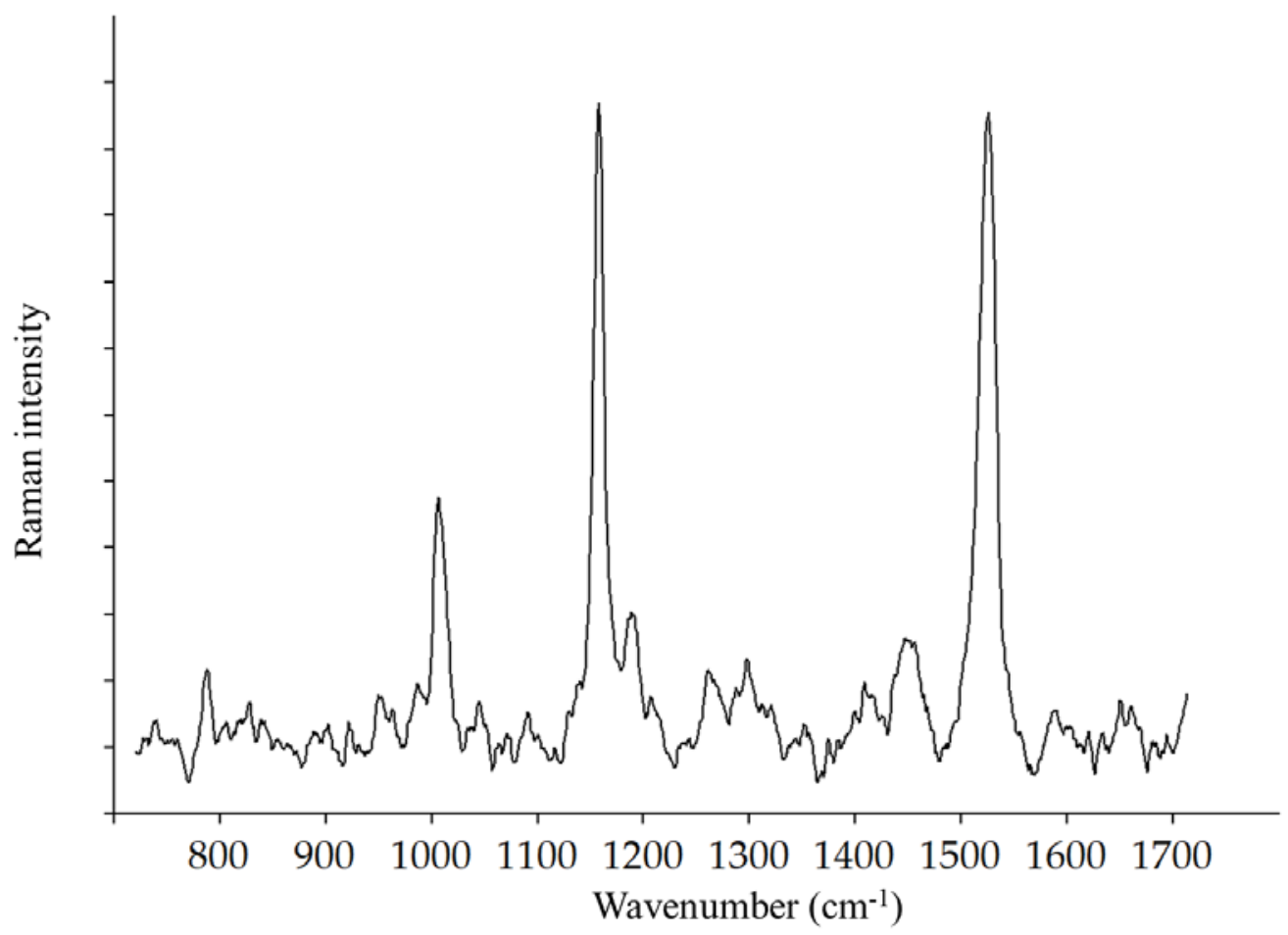

Figure 6.5. A Raman spectrum of Mycobacterium sp. MCS isolated from $5 \mu \mathrm{m}$ polystyrene spheres while trapped in the cDEP-Raman device.

The results indicate the potential of the cDEP-Raman device to decrease the analysis time of bacteria. However, Raman spectra were only collected from Mycobacterium sp. MCS. Therefore, Raman spectra from four different types of bacteria (Mycobacterium sp. MCS, Escherichia coli, Pseudomonas putida, and Streptococcus bovis) were collected using the in-house Raman microscope and compared to determine if the bacteria could be distinguished from each other. The spectra acquired from each bacterium is displayed in Figure 6.6. From observation, it is clear that distinct differences can be seen among these bacteria, which include Gram-positive, Gram-negative, and acid-fast bacteria, thus indicating the cDEP-Raman device's capability to distinguish among an array of bacteria. 


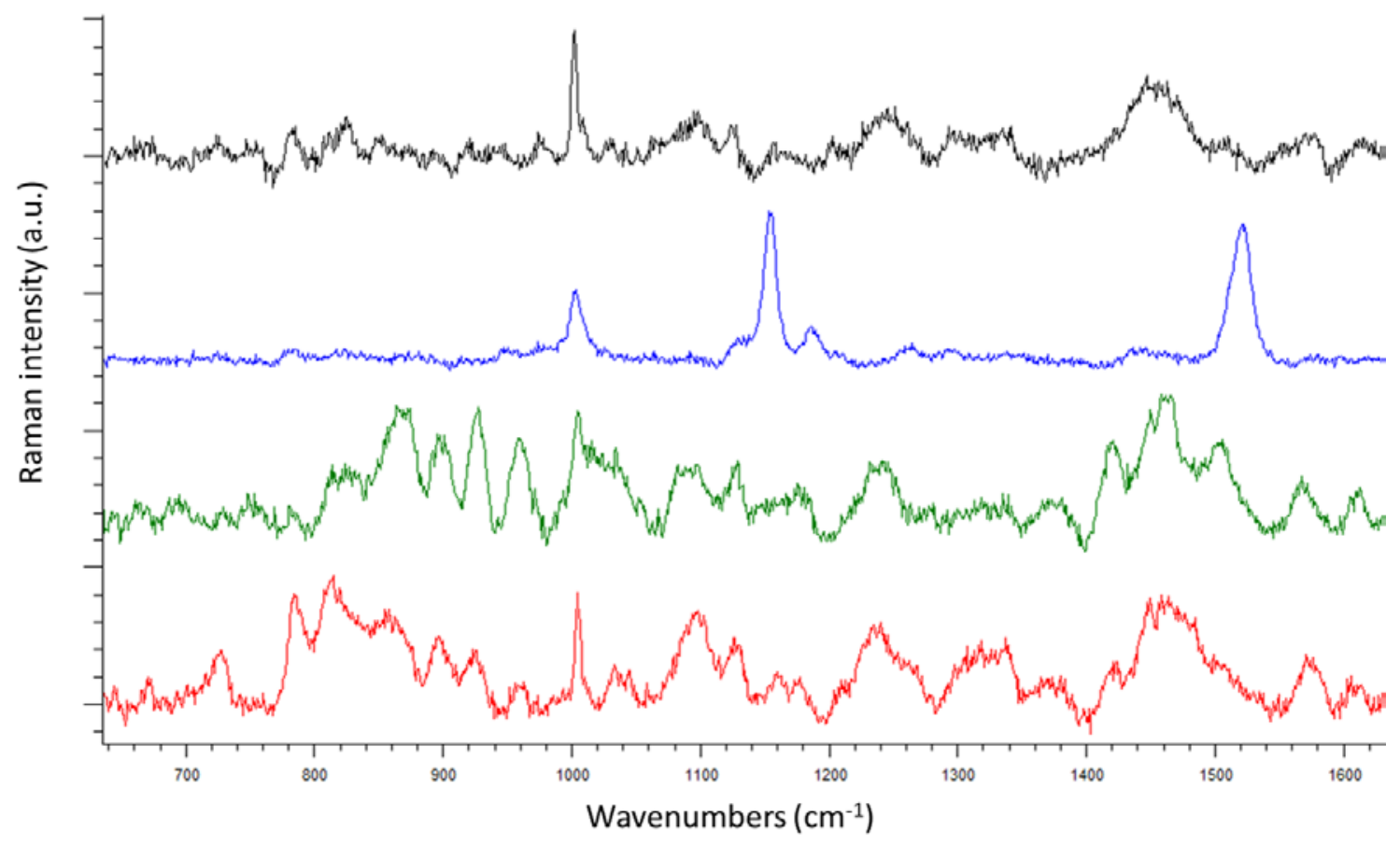

Figure 6. 6. Raman spectra of Escherichia coli (black), Mycobacterium sp. MCS (blue), Pseudomonas putida (green), and Streptococcus bovis (red).

\section{Conclusions}

In this article, we presented a cDEP-Raman device design to isolate and trap bacteria away from debris for simultaneous identification. The design is unique as the microfluidic channels are etched into fused silica rather than PDMS, allowing for the microfluidic plate to be autoclaved and reused. In addition, the device allows for the application of a non-uniform electric field to isolate bacteria from debris. The device was built and successfully tested for its capability to isolate Mycobacterium sp. MCS away from $5 \mu \mathrm{m}$ PSS spheres. In addition, spectra of Gram-negative and Gram-positive bacteria were collected using the in-house inverted Raman microscope to demonstrate the ability to identify bacteria from a wide variety of bacteria. Therefore, the cDEP-Raman design presented here has potential to decrease the analysis time of bacteria. 


\section{SUMMARY}

Bacterial identification is typically done by visualizing colonies on a series of agar plates. This method is time-consuming, taking at least one to two days for fastgrowing bacteria. For slow-growing bacteria, this process can take weeks to complete. This lengthy analysis time is the source of several problems. For example, to avoid the analysis time, doctors may improperly prescribe antibiotics without identifying the cause of an infection. Research has indicated that such improper prescription of antibiotics increases the risk of developing antibiotic-resistant strains of bacteria. Another example is in the case of emergencies or of life-threatening illnesses where one to two days for bacterial analysis is unacceptable.

To provide prompt and accurate bacterial identification, the method must not be dependent on the growth time of the bacteria, meaning that the bacteria must be identified on a microscopic scale. In addition, clinical samples will have associated debris that must be removed prior to bacterial identification. The research presented here addressed these issues by developing a device that simultaneously isolates, concentrates, and identifies bacteria using dielectrophoresis and Raman spectroscopy.

We investigated Raman spectroscopy as the means for identification, and we investigated the effect of principal component analysis (centering and scaling) on classification accuracy. Raman spectra of three closely related bacteria (Mycobacterium sp. JLS, Mycobacterium sp. MCS, and Mycobacterium sp. KMS) were collected and classified using linear discriminant analysis as well as principal component analysis. Findings indicated that centering and not scaling provided the best classification accuracies when using the cumulative percent variance method for selecting principal 
components. Among the three closely related strains, a 94.4\% classification accuracy was achieved, indicating that Raman spectroscopy is an accurate method for bacterial identification.

Raman spectroscopy provides such specific information concerning the molecular makeup of samples that even changes in bacteria growth conditions can be detected. Detectable conditions include growth phase, media, temperature, $\mathrm{pH}$, and so on. As the goal of this research is to provide prompt and accurate identification of bacteria from clinical samples, environmental factors of temperature and $\mathrm{pH}$ were not investigated, as these factors should not vary greatly from person to person. Therefore, Raman spectra of Mycobacterium sp. MCS were collected and compared according to growth phase and media. Results indicate that there are differences, which can be detected using linear discriminant analysis as well as through direct observation of spectra. Therefore, the influence of environmental growth factors must be taken into account when attempting to build a spectral library of bacteria samples for identification.

To collect Raman spectra of bacteria, a pure or highly concentrated sample is needed. Clinical samples may be from saliva, blood, urine, and sputum, which will include sample debris that must be removed to analyze bacteria. Many cell sorting methods exist to isolate bacteria, but they involve labels such as antibiotics, fluorescent tags, or primers. These labels increase costs and may result in wasted materials due to the broad range of bacteria strains that can be present in a sample. As such, label-free identification methods are appealing to cut costs and increase simplicity.

Dielectrophoresis is a label-free, cell sorting technique that uses non-uniform electric fields to cause motion in particles due to the electrical and physical properties of 
the particle and surrounding fluid. DEP is well suited for analysis of micron-sized particles. For example, DEP has been used to successfully sort bacteria away from debris and sort bacteria according to viability or antibiotic resistance. Despite DEP being a label-free and highly sensitivity technique, there are problems inherent to traditional designs, which include Joule heating, electrolysis, and electrode fouling.

Contactless dielectrophoresis is a way to implement DEP to avoid common problems of Joule heating, electrolysis, and electrode fouling. The design typically consists of a microfluidic device made of PDMS with channels for the sample and liquid electrode. The liquid electrodes are separated from the sample channel by a thin ( 20 $\mu \mathrm{m})$ insulating barrier. An AC power source is used to deliver an electric field by means of capacitive coupling. As such, the electrodes have no physical contact with the sample channel, and this, therefore, eliminates common problems associated with typical DEP methods.

Although the use of cDEP eliminates common DEP problems in operation, the method has drawbacks concerning consistent fabrication. Typical cDEP devices are made by PDMS casting, curing, and sealing to a glass slide. A poor seal anywhere along the thin $(\sim 20 \mu \mathrm{m})$ barrier between the sample and liquid electrode channels will result in an unusable device. These devices require many PDMS casts before one will work. This creates a variability from one functioning device to the next. The fabrication and operation are complicated as the insulating barrier needs to be as thin as possible for the electric field without exceeding the dielectric breakdown of PDMS. cDEP devices do avoid common DEP problems but are difficult to fabricate consistently, making it difficult to apply outside of academic settings. 
This research aimed to create a label-free device that could decrease the analysis time of bacteria using contactless dielectrophoresis and Raman spectroscopy. A unique cDEP design was developed to eliminate typical fabrication issues related to cDEP devices while retaining the advantages. The device was first modeled in COMSOL to determine if the gradient in the electric field could theoretically isolate bacteria away from debris and simultaneously trap bacteria for analysis by Raman spectroscopy. The device was then built and tested by simultaneously trapping $5 \mu \mathrm{m}$ polystyrene spheres from bacteria and collecting Raman spectra of bacteria. This successful demonstration indicates the clear potential for the use of the cDEP-Raman device in bacteria identification from clinical samples to decrease the analysis time of bacteria. 


\section{FUTURE WORK}

The goal of the research presented here was to develop a platform to decrease the analysis time of bacteria. This goal was achieved by building a novel cDEP-Raman device. The next steps of this research would be tailoring the device and related equipment for clinical applications. These changes would include testing samples that more closely mimic clinical samples, such as blood, urine, saliva, etc. In addition to hardware and sample composition, further data acquisition for a spectral library would be needed. These changes would improve isolation and identification for clinical application.

For clinical application, the microfluidic chip must be capable of trapping more than one type of bacteria and do so under a high-throughput environment. Currently the microfluidic device has only two pillar arrays, one for trapping debris and the other for trapping bacteria. To develop the device for clinical use, more arrays would be needed to trap different kinds of bacteria, as well as yeast, and trap them at faster flow rates than previously tested $(\sim 1-5 \mu \mathrm{L} / \mathrm{hr})$. Improving a chip to isolate more than one bacteria at a higher throughput would include gathering electrical and physical properties of common bacteria and COMSOL modeling to determine appropriate values of the gradient of the squared electric field as presented in Chapter 6.

Flow control of the device is also needed for clinical use. Currently, flow is gravity-controlled by monitoring the liquid levels in the inlet and outlet reservoirs. Flow could be controlled by electroosmosis by introducing a DC bias in the applied electric

field. With that setup, particles would be transported and trapped by solely electrokinetic means, eliminating the need for a syringe pump, which is commonly used in DEP 
applications. Flow control by electroosmosis would help make the cDEP device better suited for clinical samples as it would result in a more predictable flow rate that would be more easily automated.

The in-house Raman microscope could also be developed to improve identification for clinical samples. As is, there is only one operating wavelength $(785 \mathrm{~nm})$ for the in-house Raman microscope. Adding more wavelengths could improve identification capabilities, as a 2D spectrum could be acquired, as demonstrated by other researchers. Wavelengths in the ultraviolet and visible wavelength region is suggested to incorporate resonant Raman responses. For example, Raman spectra from laser wavelengths in the ultraviolet region, like $244 \mathrm{~nm}$ and $229 \mathrm{~nm}$, will primarily be rich in information about nucleic acids and aromatic amino acids, respectively [42]. Incorporating these wavelengths will require additional hardware changes such as Rayleigh filters, dichroic mirrors, and gratings with adequate quantum efficiency to collect Raman spectra. Implementing more wavelengths could improve the identification capability of the system by creating 2D spectra.

In addition to hardware changes, a larger spectral library will need to be collected and tested for the system to be ready for clinical application. The spectral library would focus on bacteria genera and species that commonly cause infections, such as Staphylococcus, Klebsiella, and Escherichia. In addition, spectra of common yeasts should be included to provide an adequate spectral library for clinical samples.

Analysis of clinical samples require identification of bacteria and antibiotic resistance in order to properly diagnosis and treat infections. Some researchers have used gradient iDEP as a means to identify antibiotic resistance. Although this is feasible, the 
use of Raman and DEP in conjunction would be preferred. Raman spectra of bacteria susceptible to antibiotics will have a different Raman signature than antibiotic-resistant bacteria before and after exposure to antibiotics. After trapping the bacteria and collecting Raman spectra, bacteria could be rinsed in the cDEP device with an antibiotic solution, and Raman spectra could be collected again. Comparison of Raman spectra before and after would discriminate between antibiotic resistant and susceptible bacteria, making the device much more applicable for clinical samples.

In order to address clinical needs, the nature of the samples tested and the sensitivity and specificity of the analysis need to be determined. The samples tested in this work included bacteria and polystyrene spheres that are roughly the same size as red blood cells (5 $\mu \mathrm{m}$ in diameter) in a low conductivity buffer. Future tests will need to be done in more realistic media such as blood, urine, and saliva. The analyses will also need to address the separation efficiency, sensitivity, and specificity to accurately compare the method against current diagnostics.

The current cDEP-Raman system is capable of simultaneously isolating and identifying bacteria, which indicates great potential for clinical applications to decrease the analysis time of bacteria. For the system to be applied in clinical settings, it must be enhanced to allow for greater automation in flow control, improved identification with more excitation wavelengths and a larger spectral library, and greater separating capability with the microfluidic plate by adding more pillar arrays. Also, focus should be placed on identifying antibiotic resistance and using media that more closely mimic reallife samples. By addressing the issues stated here, the cDEP-Raman system would be of 
great value to clinical applications for prompt and accurate diagnosis and treatment of diseases and infections. 


\section{REFERENCES}

1. Hanson, C.; Sieverts, M.; Vargis, E. Effect of principal component analysis centering and scaling on classification of mycobacteria from Raman spectra. Appl. Spectrosc. 2016, 0003702816678867, doi:10.1177/0003702816678867.

2. Hanson, C.; Vargis, E. Alternative cDEP design to facilitate cell isolation for identification by Raman spectroscopy. Sensors 2017, 17, 327, doi:10.3390/s17020327.

3. Colthup, N. B.; Daly, L. H.; Wiberley, S. E. Introduction to infrared and Raman spectroscopy; Third edition.; Academic Press: Boston, 1990; ISBN 978-0-12182554-6.

4. Analytical Raman spectroscopy; Grasselli, J. G., Bulkin, B. J., Eds.; Chemical analysis; Wiley: New York, 1991; ISBN 978-0-471-51955-3.

5. Szymanski, H. A. Raman spectroscopy: theory and practice; Plenum Press: New York, 1967;

6. Ferraro, J. R.; Nakamoto, K. Introductory Raman spectroscopy; Academic Press: Boston, 1994; ISBN 978-0-12-253990-9.

7. Eesley, G. L. Coherent Raman spectroscopy; First edition.; Pergamon Press: Oxford ; New York, 1981; ISBN 978-0-08-025058-8.

8. Aroca, R. Surface enhanced vibrational spectroscopy; Wiley: Hoboken, NJ, 2006; ISBN 978-0-471-60731-1.

9. Jones, T. B. Electromechanics of particles; Cambridge University Press: Cambridge ; New York, 1995; ISBN 978-0-521-43196-5.

10. Goddard, W. A.; Brenner, D.; Lyshevski, S. E.; Iafrate, G. J. Handbook of Nanoscience, Engineering, and Technology, Second Edition; CRC Press, 2007; ISBN 978-1-4200-0784-8.

11. Martinez-Duarte, R. Microfabrication technologies in dielectrophoresis applications-A review. Electrophoresis 2012, 33, 3110-3132, doi:10.1002/elps.201200242.

12. Li, M.; Li, W. H.; Zhang, J.; Alici, G.; Wen, W. A review of microfabrication techniques and dielectrophoretic microdevices for particle manipulation and separation. J. Phys. -Appl. Phys. 2014, 47, 063001, doi:10.1088/00223727/47/6/063001.

13. Pethig, R. Review article-dielectrophoresis: status of the theory, technology, and applications. Biomicrofluidics 2010, 4, 022811, doi:10.1063/1.3456626.

14. Srivastava, S. K.; Gencoglu, A.; Minerick, A. R. DC insulator dielectrophoretic applications in microdevice technology: a review. Anal. Bioanal. Chem. 2011, 399, 301-321, doi:10.1007/s00216-010-4222-6.

15. Yang, L. A review of multifunctions of dielectrophoresis in biosensors and biochips for bacteria detection. Anal. Lett. 2012, 45, 187-201, doi:10.1080/00032719.2011.633182.

16. Baudelet, M.; Guyon, L.; Yu, J.; Wolf, J.-P.; Amodeo, T.; Fréjafon, E.; Laloi, P. Femtosecond time-resolved laser-induced breakdown spectroscopy for detection and identification of bacteria: A comparison to the nanosecond regime. J. Appl. Phys. 2006, 99, 084701, doi:10.1063/1.2187107. 
17. Marcos-Martinez, D.; Ayala, J. A.; Izquierdo-Hornillos, R. C.; de Villena, F. J. M.; Caceres, J. O. Identification and discrimination of bacterial strains by laser induced breakdown spectroscopy and neural networks. Talanta 2011, 84, 730-737, doi:10.1016/j.talanta.2011.01.069.

18. Manzoor, S.; Moncayo, S.; Navarro-Villoslada, F.; Ayala, J. A.; IzquierdoHornillos, R.; de Villena, F. J. M.; Caceres, J. O. Rapid identification and discrimination of bacterial strains by laser induced breakdown spectroscopy and neural networks. Talanta 2014, 121, 65-70, doi:10.1016/j.talanta.2013.12.057.

19. Wehrli, P. M.; Lindberg, E.; Svensson, O.; Sparen, A.; Josefson, M.; Dunstan, R. H.; Wold, A. E.; Gottfries, J. Exploring bacterial phenotypic diversity using factorial design and FTIR multivariate fingerprinting. J. Chemom. 2014, 28, 681686, doi:10.1002/cem.2588.

20. Zarnowiec, P.; Lechowicz, L.; Czerwonka, G.; Kaca, W. Fourier transform infrared spectroscopy (FTIR) as a tool for the identification and differentiation of pathogenic bacteria. Curr. Med. Chem. 2015, 22, 1710-1718.

21. Elsayeh, M.; Kandil, A. H. Detection and identification system of bacteria and bacterial endotoxin based on Raman spectroscopy. Int. J. Adv. Comput. Sci. Appl. 2016, 7, 201-205.

22. Giana, H. E.; Silveira, L.; Zângaro, R. A.; Pacheco, M. T. T. Rapid identification of bacterial species by fluorescence spectroscopy and classification through principal components analysis. J. Fluoresc. 2003, 13, 489-493, doi:10.1023/B:JOFL.0000008059.74052.3c.

23. Molyneux, P. M.; Kilvington, S.; Wakefield, M. J.; Prydal, J. I.; Bannister, N. P. Autofluorescence signatures of seven pathogens: preliminary in vitro investigations of a potential diagnostic for Acanthamoeba keratitis. Cornea 2015, 34, 1588-1592, doi:10.1097/ICO.0000000000000645.

24. Webb, S.; Stoneham, M. Resonances between 1011 and $1012 \mathrm{~Hz}$ in active bacterial-cells as seen by laser Raman-spectroscopy. Phys. Lett. A 1977, 60, 267268, doi:10.1016/0375-9601(77)90841-6.

25. Webb, S. J. Laser-Raman spectroscopy of living cells. Phys. Rep. 1980, 60, 201224, doi:10.1016/0370-1573(80)90149-0.

26. Webb, S.; Stoneham, M.; Frohlich, H. Evidence for nonthermal excitation of energy-levels in active biological-systems. Phys. Lett. A 1977, 63, 407-408, doi:10.1016/0375-9601(77)90948-3.

27. Layne, S. P.; Bigio, I. J. Raman spectroscopy of Bacillus megaterium using an optical multi-channel analyzer. Phys. Scr. 1986, 33, 91, doi:10.1088/00318949/33/1/014.

28. Furia, L.; Gandhi, O. P. Absence of biologically related Raman lines in cultures of Bacillus megaterium. Phys. Lett. A 1984, 102, 380-382, doi:10.1016/03759601(84)90304-9.

29. Hutsebaut, D.; Maquelin, K.; De Vos, P.; Vandenabeele, P.; Moens, L.; Puppels, G. J. Effect of culture conditions on the achievable taxonomic resolution of Raman spectroscopy disclosed by three Bacillus species. Anal. Chem. 2004, 76, 62746281, doi:10.1021/ac0492281.

30. Harz, M.; Rosch, P.; Peschke, K. D.; Ronneberger, O.; Burkhardt, H.; Popp, J. Micro-Raman spectroscopic identification of bacterial cells of the genus 
Staphylococcus and dependence on their cultivation conditions. Analyst 2005, 130, 1543-1550, doi:10.1039/b507715j.

31. Escoriza, M. F.; Vanbriesen, J. M.; Stewart, S.; Maier, J. Studying bacterial metabolic states using Raman spectroscopy. Appl. Spectrosc. 2006, 60, 971-976, doi:10.1366/000370206778397290.

32. Espagnon, I.; Ostrovskii, D.; Mathey, R.; Dupoy, M.; Joly, P. L.; NovelliRousseau, A.; Pinston, F.; Gal, O.; Mallard, F.; Leroux, D. F. Direct identification of clinically relevant bacterial and yeast microcolonies and macrocolonies on solid culture media by Raman spectroscopy. J. Biomed. Opt. 2014, 19, 027004, doi:10.1117/1.JBO.19.2.027004.

33. Kunapareddy, N.; Grun, J.; Lunsford, R.; Nikitin, S.; Wang, Z.; Gillis, D. Multiwavelength resonance Raman characterization of the effect of growth phase and culture medium on bacteria. Appl. Spectrosc. 2015, 69, 966-971, doi:10.1366/14-07770.

34. Choo-Smith, L. P.; Maquelin, K.; van Vreeswijk, T.; Bruining, H. A.; Puppels, G. J.; Thi, N. a. G.; Kirschner, C.; Naumann, D.; Ami, D.; Villa, A. M.; Orsini, F.; Doglia, S. M.; Lamfarraj, H.; Sockalingum, G. D.; Manfait, M.; Allouch, P.; Endtz, H. P. Investigating microbial (micro)colony heterogeneity by vibrational spectroscopy. Appl. Environ. Microbiol. 2001, 67, 1461-1469, doi:10.1128/AEM.67.4.1461-1469.2001.

35. Xie, C.; Mace, J.; Dinno, M. A.; Li, Y. Q.; Tang, W.; Newton, R. J.; Gemperline, $\mathrm{P}$. J. Identification of single bacterial cells in aqueous solution using confocal laser tweezers Raman spectroscopy. Anal. Chem. 2005, 77, 4390-4397, doi:10.1021/ac0504971.

36. Moritz, T. J.; Taylor, D. S.; Polage, C. R.; Krol, D. M.; Lane, S. M.; Chan, J. W. Effect of Cefazolin treatment on the nonresonant Raman signatures of the metabolic state of individual Escherichia coli cells. Anal. Chem. 2010, 82, 27032710, doi:10.1021/ac902351a.

37. Stöckel, S.; Stanca, A. S.; Helbig, J.; Rösch, P.; Popp, J. Raman spectroscopic monitoring of the growth of pigmented and non-pigmented mycobacteria. Anal. Bioanal. Chem. 2015, 407, 8919-8923, doi:10.1007/s00216-015-9031-5.

38. Huang, W. E.; Griffiths, R. I.; Thompson, I. P.; Bailey, M. J.; Whiteley, A. S. Raman microscopic analysis of single microbial cells. Anal. Chem. 2004, 76, 4452-4458, doi:10.1021/ac049753k.

39. Mlynáriková, K.; Samek, O.; Bernatová, S.; Růžička, F.; Ježek, J.; Hároniková, A.; Šiler, M.; Zemánek, P.; Holá, V. Influence of culture media on microbial fingerprints using Raman spectroscopy. Sensors 2015, 15, 29635-29647, doi:10.3390/s151129635.

40. Stephen, K. E.; Homrighausen, D.; DePalma, G.; Nakatsu, C. H.; Irudayaraj, J. Surface enhanced Raman spectroscopy (SERS) for the discrimination of Arthrobacter strains based on variations in cell surface composition. Analyst 2012, 137, 4280-4286, doi:10.1039/c2an35578g.

41. Premasiri, W. R.; Gebregziabher, Y.; Ziegler, L. D. On the difference between SERS spectra of cell growth media and whole bacterial cells. Appl. Spectrosc. 2011, 65, 493-499, doi:10.1366/10-06173. 
42. Jarvis, R. M.; Goodacre, R. Ultra-violet resonance Raman spectroscopy for the rapid discrimination of urinary tract infection bacteria. Fems Microbiol. Lett. 2004, 232, 127-132, doi:10.1016/S0378-1097(04)00040-0.

43. Sung, N.; Collins, M. T. Variation in resistance of Mycobacterium paratuberculosis to acid environments as a function of culture medium. Appl. Environ. Microbiol. 2003.

44. Balážová, T.; Makovcová, J.; Šedo, O.; Slaný, M.; Faldyna, M.; Zdráhal, Z. The influence of culture conditions on the identification of Mycobacterium species by MALDI-TOF MS profiling. FEMS Microbiol. Lett. 2014, 353, 77-84, doi:10.1111/1574-6968.12408.

45. Kumar, S.; Matange, N.; Umapathy, S.; Visweswariah, S. S. Linking carbon metabolism to carotenoid production in mycobacteria using Raman spectroscopy. FEMS Microbiol. Lett. 2015, 362, 1-6, doi:10.1093/femsle/fnu048.

46. Buijtels, P. C. A. M.; Willemse-Erix, H. F. M.; Petit, P. L. C.; Endtz, H. P.; Puppels, G. J.; Verbrugh, H. A.; van Belkum, A.; van Soolingen, D.; Maquelin, K. Rapid identification of mycobacteria by Raman spectroscopy. J. Clin. Microbiol. 2008, 46, 961-965, doi:10.1128/JCM.01763-07.

47. Rivera-Betancourt, O. E.; Karls, R.; Grosse-Siestrup, B.; Helms, S.; Quinn, F.; Dluhy, R. A. Identification of mycobacteria based on spectroscopic analyses of mycolic acid profiles. The Analyst 2013, 138, 6774, doi:10.1039/c3an01157g.

48. Stöckel, S.; Meisel, S.; Lorenz, B.; Kloß, S.; Henk, S.; Dees, S.; Richter, E.; Andres, S.; Merker, M.; Labugger, I.; Rösch, P.; Popp, J. Raman spectroscopic identification of Mycobacterium tuberculosis. J. Biophotonics 2017, 10, 727-734, doi:10.1002/jbio.201600174.

49. Mühlig, A.; Bocklitz, T.; Labugger, I.; Dees, S.; Henk, S.; Richter, E.; Andres, S.; Merker, M.; Stöckel, S.; Weber, K.; Cialla-May, D.; Popp, J. LOC-SERS: A promising closed system for the identification of mycobacteria. Anal. Chem. 2016, 88, 7998-8004, doi:10.1021/acs.analchem.6b01152.

50. Tang, M.; McEwen, G. D.; Wu, Y.; Miller, C. D.; Zhou, A. Characterization and analysis of mycobacteria and Gram-negative bacteria and co-culture mixtures by Raman microspectroscopy, FTIR, and atomic force microscopy. Anal. Bioanal. Chem. 2013, 405, 1577-1591, doi:10.1007/s00216-012-6556-8.

51. Imai, T.; Urushiyama, A.; Saito, H.; Sakamoto, Y.; Ota, K.; Ohmori, D. A novel 6Fe (2 $\times$ [3Fe-4S]) ferredoxin from Mycobacterium smegmatis. FEBS Lett. 1995, 368, 23-26, doi:10.1016/0014-5793(95)00601-5.

52. Wengenack, N. L.; Lane, B. D.; Hill, P. J.; Uhl, J. R.; Lukat-Rodgers, G. S.; Hall, L.; Roberts, G. D.; Cockerill, F. R.; Brennan, P. J.; Rodgers, K. R.; Belisle, J. T.; Rusnak, F. Purification and characterization of Mycobacterium tuberculosis KatG, KatG(S315T), and Mycobacterium bovis KatG(R463L). Protein Expr. Purif. 2004, 36, 232-243, doi:10.1016/j.pep.2004.04.018.

53. Kapetanaki, S. M.; Zhao, X.; Yu, S.; Magliozzo, R. S.; Schelvis, J. P. M. Modification of the active site of Mycobacterium tuberculosis KatG after disruption of the Met-Tyr-Trp cross-linked adduct. J. Inorg. Biochem. 2007, 101, 422-433, doi:10.1016/j.jinorgbio.2006.11.004.

54. McLean, K. J.; Cheesman, M. R.; Rivers, S. L.; Richmond, A.; Leys, D.; Chapman, S. K.; Reid, G. A.; Price, N. C.; Kelly, S. M.; Clarkson, J.; Smith, W. 
E.; Munro, A. W. Expression, purification and spectroscopic characterization of the cytochrome P450 CYP121 from Mycobacterium tuberculosis. J. Inorg. Biochem. 2002, 91, 527-541, doi:10.1016/S0162-0134(02)00479-8.

55. Pohl, H. A. The motion and precipitation of suspensoids in divergent electric fields. J. Appl. Phys. 1951, 22, 869-871, doi:10.1063/1.1700065.

56. Allahrabbi, N.; Chia, Y. S. M.; Saifullah, M. S. M.; Lim, K.-M.; Yung, L. Y. L. A hybrid dielectrophoretic system for trapping of microorganisms from water. Biomicrofluidics 2015, 9, 034110, doi:10.1063/1.4922276.

57. Lapizco-Encinas, B. H.; Simmons, B. A.; Cummings, E. B.; Fintschenko, Y. Insulator-based dielectrophoresis for the selective concentration and separation of live bacteria in water. Electrophoresis 2004, 25, 1695-1704, doi:10.1002/elps.200405899.

58. Lapizco-Encinas, B. H.; Davalos, R. V.; Simmons, B. A.; Cummings, E. B.; Fintschenko, Y. An insulator-based (electrodeless) dielectrophoretic concentrator for microbes in water. J. Microbiol. Methods 2005, 62, 317-326, doi:10.1016/j.mimet.2005.04.027.

59. Wang, X.-B.; Huang, Y.; Burt, J. P. H.; Markx, G. H.; Pethig, R. Selective dielectrophoretic confinement of bioparticles in potential energy wells. J. Phys. Appl. Phys. 1993, 26, 1278-1285, doi:10.1088/0022-3727/26/8/019.

60. Cheng, J.; Sheldon, E. L.; Wu, L.; Uribe, A.; Gerrue, L. O.; Carrino, J.; Heller, M. J.; O'Connell, J. P. Preparation and hybridization analysis of DNA/RNA from Ecoli on microfabricated bioelectronic chips. Nat. Biotechnol. 1998, 16, 541-546, doi:10.1038/nbt0698-541.

61. Cheng, I.-F.; Lin, C.-C.; Lin, D.-Y.; Chang, H.- A dielectrophoretic chip with a roughened metal surface for on-chip surface-enhanced Raman scattering analysis of bacteria. Biomicrofluidics 2010, 4, 034104, doi:10.1063/1.3474638.

62. Markx, G.; Huang, Y.; Zhou, X.; Pethig, R. Dielectrophoretic characterization and separation of microorganisms. Microbiol.-Uk 1994, 140, 585-591.

63. Markx, G.; Pethig, R. Dielectrophoretic separation of cells: continuous separation. Biotechnol. Bioeng. 1995, 45, 337-343, doi:10.1002/bit.260450408.

64. Moncada-Hernandez, H.; Lapizco-Encinas, B. H. Simultaneous concentration and separation of microorganisms: insulator-based dielectrophoretic approach. Anal. Bioanal. Chem. 2010, 396, 1805-1816, doi:10.1007/s00216-009-3422-4.

65. Moncada-Hernandez, H.; Baylon-Cardiel, J. L.; Perez-Gonzalez, V. H.; LapizcoEncinas, B. H. Insulator-based dielectrophoresis of microorganisms: theoretical and experimental results. Electrophoresis 2011, 32, 2502-2511, doi:10.1002/elps.201100168.

66. Khoshmanesh, K.; Baratchi, S.; Tovar-Lopez, F. J.; Nahavandi, S.; Wlodkowic, D.; Mitchell, A.; Kalantar-zadeh, K. On-chip separation of Lactobacillus bacteria from yeasts using dielectrophoresis. Microfluid. Nanofluidics 2012, 12, 597-606, doi:10.1007/s10404-011-0900-8.

67. Van den Driesche, S.; Rao, V.; Puchberger-Enengl, D.; Witarski, W.; Vellekoop, M. J. Continuous cell from cell separation by traveling wave dielectrophoresis. Sens. Actuators B Chem. 2012, 170, 207-214, doi:10.1016/j.snb.2011.01.012. 
68. Lapizco-Encinas, B. H.; Simmons, B. A.; Cummings, E. B.; Fintschenko, Y. Dielectrophoretic concentration and separation of live and dead bacteria in an array of insulators. Anal. Chem. 2004, 76, 1571-1579, doi:10.1021/ac034804j.

69. Fatoyinbo, H. O.; Hughes, M. P.; Martin, S. P.; Pashby, P.; Labeed, F. H. Dielectrophoretic separation of Bacillus subtilis spores from environmental diesel particles. J. Environ. Monit. 2007, 9, 87-90, doi:10.1039/b614556f.

70. Moon, H.-S.; Nam, Y.-W.; Park, J. C.; Jung, H.-I. Dielectrophoretic separation of airborne microbes and dust particles using a microfluidic channel for real-time bioaerosol monitoring. Environ. Sci. Technol. 2009, 43, 5857-5863, doi:10.1021/es900078z.

71. Cheng, I.-F.; Chang, H.-C.; Hou, D.; Chang, H.-C. An integrated dielectrophoretic chip for continuous bioparticle filtering, focusing, sorting, trapping, and detecting. Biomicrofluidics 2007, 1, 021503, doi:10.1063/1.2723669.

72. Chow, K.-S.; Du, H. Dielectrophoretic characterization and trapping of different waterborne pathogen in continuous flow manner. Sens. Actuators Phys. 2011, 170, 24-31, doi:10.1016/j.sna.2011.03.053.

73. Lewpiriyawong, N.; Kandaswamy, K.; Yang, C.; Ivanov, V.; Stocker, R. Microfluidic characterization and continuous separation of cells and particles using conducting poly(dimethyl siloxane) electrode induced alternating currentdielectrophoresis. Anal. Chem. 2011, 83, 9579-9585, doi:10.1021/ac202137y.

74. Barrett, L. M.; Skulan, A. J.; Singh, A. K.; Cummings, E. B.; Fiechtner, G. J. Dielectrophoretic manipulation of particles and cells using insulating ridges in faceted prism microchannels. Anal. Chem. 2005, 77, 6798-6804, doi:10.1021/ac0507791.

75. Markx, G. H.; Dyda, P. A.; Pethig, R. Dielectrophoretic separation of bacteria using a conductivity gradient. J. Biotechnol. 1996, 51, 175-180, doi:10.1016/01681656(96)01617-3.

76. Jaramillo, M. del C.; Torrents, E.; Martínez-Duarte, R.; Madou, M. J.; Juárez, A. On-line separation of bacterial cells by carbon-electrode dielectrophoresis. Electrophoresis 2010, 31, 2921-2928, doi:10.1002/elps.201000082.

77. Jones, P. V.; DeMichele, A. F.; Kemp, L.; Hayes, M. A. Differentiation of Escherichia coli serotypes using DC gradient insulator dielectrophoresis. Anal. Bioanal. Chem. 2014, 406, 183-192, doi:10.1007/s00216-013-7437-5.

78. Suehiro, J.; Hamada, R.; Noutomi, D.; Shutou, M.; Hara, M. Selective detection of viable bacteria using dielectrophoretic impedance measurement method. $J$. Electrost. 2003, 57, 157-168, doi:10.1016/S0304-3886(02)00124-9.

79. Pysher, M. D.; Hayes, M. A. Electrophoretic and dielectrophoretic field gradient technique for separating bioparticles. Anal. Chem. 2007, 79, 4552-4557, doi:10.1021/ac070534j.

80. Chung, C.-C.; Cheng, I.-F.; Yang, W.-H.; Chang, H.-C. Antibiotic susceptibility test based on the dielectrophoretic behavior of elongated Escherichia coli with cephalexin treatment. Biomicrofluidics 2011, 5, 021102, doi:10.1063/1.3600650.

81. Chung, C.-C.; Cheng, I.-F.; Chen, H.-M.; Kan, H.-C.; Yang, W.-H.; Chang, H.-C. Screening of antibiotic susceptibility to $\beta$-lactam-induced elongation of gramnegative bacteria based on dielectrophoresis. Anal. Chem. 2012, 84, 3347-3354, doi:10.1021/ac300093w. 
82. Hoettges, K. F.; Dale, J. W.; Hughes, M. P. Rapid determination of antibiotic resistance in E. coli using dielectrophoresis. Phys. Med. Biol. 2007, 52, 60016009, doi:10.1088/0031-9155/52/19/019.

83. Jones, P. V.; Huey, S.; Davis, P.; McLemore, R.; McLaren, A.; Hayes, M. A. Biophysical separation of Staphylococcus epidermidis strains based on antibiotic resistance. Analyst 2015, 140, 5152-5161, doi:10.1039/C5AN00906E.

84. Elitas, M.; Martinez-Duarte, R.; Dhar, N.; McKinney, J. D.; Renaud, P. Dielectrophoresis-based purification of antibiotic-treated bacterial subpopulations. Lab. Chip 2014, 14, 1850-1857, doi:10.1039/c4lc00109e.

85. Suehiro, J.; Ohtsubo, A.; Hatano, T.; Hara, M. Selective detection of bacteria by a dielectrophoretic impedance measurement method using an antibody-immobilized electrode chip. Sens. Actuators B-Chem. 2006, 119, 319-326, doi:10.1016/j.snb.2005.12.027.

86. Lagally, E. T.; Lee, S. H.; Soh, H. T. Integrated microsystem for dielectrophoretic cell concentration and genetic detection. Lab. Chip 2005, 5, 1053-1058, doi:10.1039/b505915a.

87. Moon, H.-S.; Im, H. T.; Choi, A.; Jung, H.-I. Real-time detection of food-borne bacterial adenosine triphosphate (ATP) using dielectrophoretic force and a bioluminescence sensor. Microchim. Acta 2010, 170, 283-288, doi:10.1007/s00604-010-0370-9.

88. Sabounchi, P.; Morales, A. M.; Ponce, P.; Lee, L. P.; Simmons, B. A.; Davalos, R. V. Sample concentration and impedance detection on a microfluidic polymer chip. Biomed. Microdevices 2008, 10, 661-670, doi:10.1007/s10544-008-9177-4.

89. Dastider, S. G.; Barizuddin, S.; Dweik, M.; Almasri, M. A micromachined impedance biosensor for accurate and rapid detection of E. coli O157:H7. Rsc Adv. 2013, 3, 26297-26306, doi:10.1039/c3ra44724c.

90. Couniot, N.; Francis, L. A.; Flandre, D. Resonant dielectrophoresis and electrohydrodynamics for high-sensitivity impedance detection of whole-cell bacteria. Lab. Chip 2015, 15, 3183-3191, doi:10.1039/c5lc00090d.

91. Kim, M.; Jung, T.; Kim, Y.; Lee, C.; Woo, K.; Seol, J. H.; Yang, S. A microfluidic device for label-free detection of Escherichia coli in drinking water using positive dielectrophoretic focusing, capturing, and impedance measurement. Biosens. Bioelectron. 2015, 74, 1011-1015, doi:10.1016/j.bios.2015.07.059.

92. Schröder, U.-C.; Ramoji, A.; Glaser, U.; Sachse, S.; Leiterer, C.; Csaki, A.; Hübner, U.; Fritzsche, W.; Pfister, W.; Bauer, M.; Popp, J.; Neugebauer, U. Combined dielectrophoresis-Raman setup for the classification of pathogens recovered from the urinary tract. Anal. Chem. 2013, 85, 10717-10724, doi:10.1021/ac4021616.

93. Schröder, U.-C.; Beleites, C.; Assmann, C.; Glaser, U.; Huebner, U.; Pfister, W.; Fritzsche, W.; Popp, J.; Neugebauer, U. Detection of vancomycin resistances in enterococci within 3 1/2 hours. Sci. Rep. 2015, 5, 8217, doi:10.1038/srep08217.

94. Cheng, I.-F.; Chen, T.-Y.; Lu, R.-J.; Wu, H.-W. Rapid identification of bacteria utilizing amplified dielectrophoretic force-assisted nanoparticle-induced surfaceenhanced Raman spectroscopy. Nanoscale Res. Lett. 2014, 9, 1-8, doi:10.1186/1556-276X-9-324. 
95. Lin, H.-Y.; Huang, C.-H.; Hsieh, W.-H.; Liu, L.-H.; Lin, Y.-C.; Chu, C.-C.; Wang, S.-T.; Kuo, I.-T.; Chau, L.-K.; Yang, C.-Y. On-line SERS detection of single bacterium using novel SERS nanoprobes and a microfluidic dielectrophoresis device. Small 2014, 10, 4700-4710, doi:10.1002/smll.201401526.

96. Madiyar, F. R.; Bhana, S.; Swisher, L. Z.; Culbertson, C. T.; Huang, X.; Li, J. Integration of a nanostructured dielectrophoretic device and a surface-enhanced Raman probe for highly sensitive rapid bacteria detection. Nanoscale 2015, 7, 3726-3736, doi:10.1039/c4nr07183b.

97. Cheng, I.-F.; Chang, H.-C.; Chen, T.-Y.; Hu, C.; Yang, F.-L. Rapid (< 5 min) identification of pathogen in human blood by electrokinetic concentration and surface-enhanced Raman spectroscopy. Sci. Rep. 2013, 3, 2365, doi:10.1038/srep02365.

98. Maquelin, K.; Kirschner, C.; Choo-Smith, L.-P.; Ngo-Thi, N. A.; Vreeswijk, T. van; Stämmler, M.; Endtz, H. P.; Bruining, H. A.; Naumann, D.; Puppels, G. J. Prospective study of the performance of vibrational spectroscopies for rapid identification of bacterial and fungal pathogens recovered from blood cultures. $J$. Clin. Microbiol. 2003, 41, 324-329, doi:10.1128/JCM.41.1.324-329.2003.

99. Amiel, C.; Mariey, L.; Curk-Daubie, M. C.; Pichon, P.; Travert, J. Potentiality of Fourier Transform Infrared Spectroscopy (FTIR) for discrimination and identification of dairy Lactic acid bacteria. Lait 2000, 80, 445-459.

100. Escoriza, M. F.; VanBriesen, J. M.; Stewart, S.; Maier, J.; Treado, P. J. Raman spectroscopy and chemical imaging for quantification of filtered waterborne bacteria. J. Microbiol. Methods 2006, 66, 63-72, doi:10.1016/j.mimet.2005.10.013.

101. Guicheteau, J.; Argue, L.; Emge, D.; Hyre, A.; Jacobson, M.; Christesen, S. Bacillus spore classification via surface-enhanced Raman spectroscopy and principal component analysis. Appl. Spectrosc. 2008, 62, 267-272, doi:10.1366/000370208783759623.

102. Parisi, D.; Magliulo, M.; Nanni, P.; Casale, M.; Forina, M.; Roda, A. Analysis and classification of bacteria by matrix-assisted laser desorption/ionization time-offlight mass spectrometry and a chemometric approach. Anal. Bioanal. Chem. 2008, 391, 2127-2134, doi:10.1007/s00216-008-2161-2.

103. De Bruyne, K.; Slabbinck, B.; Waegeman, W.; Vauterin, P.; De Baets, B.; Vandamme, P. Bacterial species identification from MALDI-TOF mass spectra through data analysis and machine learning. Syst. Appl. Microbiol. 2011, 34, 2029, doi:10.1016/j.syapm.2010.11.003.

104. Foster, N. S.; Thompson, S. E.; Valentine, N. B.; Amonette, J. E.; Johnson, T. J. Identification of sporulated and vegetative bacteria using statistical analysis of Fourier transform mid-infrared transmission data. Appl. Spectrosc. 2004, 58, 203211, doi:10.1366/000370204322842940.

105. Samuels, A. C.; Snyder, A. P.; Emge, D. K.; Amant, D. S.; Minter, J.; Campbell, M.; Tripathi, A. Classification of select Category A and B bacteria by Fourier transform infrared spectroscopy. Appl. Spectrosc. 2009, 63, 14-24.

106. Schmid, U.; Roesch, P.; Krause, M.; Harz, M.; Popp, J.; Baumann, K. Gaussian mixture discriminant analysis for the single-cell differentiation of bacteria using 
micro-Raman spectroscopy. Chemom. Intell. Lab. Syst. 2009, 96, 159-171, doi:10.1016/j.chemolab.2009.01.008.

107. Jolliffe, I. T. Principal component analysis; Springer series in statistics; 2nd ed.; Springer: New York, 2002;

108. Dunteman, G. H. Principal components analysis; Quantitative applications in the social sciences; SAGE: Newbury Park, [Calif.] ; London, 1989; ISBN 978-1-41298547-5.

109. Cangelosi, R.; Goriely, A. Component retention in principal component analysis with application to cDNA microarray data. Biol. Direct 2007, 2, UNSP 2, doi:10.1186/1745-6150-2-2.

110. Home - Mycobacterium sp. JLS Available online: http://genome.jgi.doe.gov/myc_j/myc_j.home.html (accessed on Aug 8, 2016).

111. Home - Mycobacterium sp. KMS Available online: http://genome.jgi.doe.gov/myc_k/myc_k.home.html (accessed on Aug 8, 2016).

112. Home - Mycobacterium sp. MCS Available online: http://genome.jgi.doe.gov/myc_m/myc_m.home.html (accessed on Aug 8, 2016).

113. De Gelder, J.; Scheldeman, P.; Leus, K.; Heyndrickx, M.; Vandenabeele, P.; Moens, L.; De Vos, P. Raman spectroscopic study of bacterial endospores. Anal. Bioanal. Chem. 2007, 389, 2143-2151, doi:10.1007/s00216-007-1616-1.

114. Menges, F. Spekwin32 - optical spectrscopy software;

115. Varmuza, K.; Filzmoser, P. Introduction to multivariate statistical analysis in chemometrics; CRC Press: Boca Raton, 2009; ISBN 978-1-4200-5947-2.

116. Howley, T.; Madden, M. G.; O’Connell, M.-L.; Ryder, A. G. The effect of principal component analysis on machine learning accuracy with high-dimensional spectral data. Knowl.-Based Syst. 2006, 19, 363-370, doi:10.1016/j.knosys.2005.11.014.

117. Mycobacterium sp. MCS (ID 13563) - Genome - NCBI Available online: http://www.ncbi.nlm.nih.gov/genome/13563?genome_assembly_id=174992 (accessed on Aug 10, 2016).

118. Schuster, K. C.; Urlaub, E.; Gapes, J. R. Single-cell analysis of bacteria by Raman microscopy: spectral information on the chemical composition of cells and on the heterogeneity in a culture. J. Microbiol. Methods 2000, 42, 29-38, doi:10.1016/S0167-7012(00)00169-X.

119. Manoharan, R.; Ghiamati, E.; Chadha, S.; Nelson, W. H.; Sperry, J. F. Effect of cultural conditions on deep UV resonance Raman spectra of bacteria. Appl. Spectrosc. 1993, 47, 2145-2150.

120. WHO | Global tuberculosis report 2017 Available online: http://www.who.int/tb/publications/global_report/en/ (accessed on Feb 14, 2018).

121. Talukder, A.; Ishihama, A. Growth phase dependent changes in the structure and protein composition of nucleoid in Escherichia coli. Sci. China Life Sci. 2015, 58, 902-911, doi:10.1007/s11427-015-4898-0.

122. Muffler, A.; Fischer, D.; Hengge-Aronis, R. The RNA-binding protein HF-I, known as a host factor for phage Qbeta RNA replication, is essential for rpoS translation in Escherichia coli. Genes Dev. 1996, 10, 1143-1151. 
123. Schuppli, D.; Miranda, G.; Tsui, H.-C. T.; Winkler, M. E.; Sogo, J. M.; Weber, H. Altered 3'-terminal RNA structure in phage $\mathrm{Q} \beta$ adapted to host factor-less Escherichia coli. Proc. Natl. Acad. Sci. U. S. A. 1997, 94, 10239-10242.

124. Boubrik, F.; Rouviere-Yaniv, J. Increased sensitivity to gamma irradiation in bacteria lacking protein HU. Proc. Natl. Acad. Sci. U. S. A. 1995, 92, 3958-3962.

125. Dri, A. M.; Moreau, P. L.; Rouvière-Yaniv, J. Role of the histone-like proteins OsmZ and HU in homologous recombination. Gene 1992, 120, 11-16.

126. Jaffe, A.; Vinella, D.; D’Ari, R. The Escherichia coli histone-like protein HU affects DNA initiation, chromosome partitioning via MukB, and cell division via MinCDE. J. Bacteriol. 1997, 179, 3494-3499, doi:10.1128/jb.179.11.34943499.1997.

127. Kano, Y.; Goshima, N.; Wada, M.; Imamoto, F. Participation of hup gene product in replicative transposition of $\mathrm{Mu}$ phage in Escherichia coli. Gene 1989, 76, 353358.

128. Drlica, K.; Rouviere-Yaniv, J. Histonelike proteins of bacteria. Microbiol. Rev. 1987, 51, 301-319.

129. Azam, T. A.; Iwata, A.; Nishimura, A.; Ueda, S.; Ishihama, A. Growth phasedependent variation in protein composition of the Escherichia coli nucleoid. $J$. Bacteriol. 1999, 181, 6361-6370.

130. Nair, S.; Finkel, S. E. Dps protects cells against multiple stresses during stationary phase. J. Bacteriol. 2004, 186, 4192-4198, doi:10.1128/JB.186.13.41924198.2004.

131. Gagnon, Z. R. Cellular dielectrophoresis: Applications to the characterization, manipulation, separation and patterning of cells. Electrophoresis 2011, 32, 24662487, doi:10.1002/elps.201100060.

132. Shafiee, H.; Caldwell, J. L.; Sano, M. B.; Davalos, R. V. Contactless dielectrophoresis: a new technique for cell manipulation. Biomed. Microdevices 2009, 11, 997-1006, doi:10.1007/s10544-009-9317-5.

133. Hughes, M. P. Strategies for dielectrophoretic separation in laboratory-on-a-chip systems. Electrophoresis 2002, 23, 2569-2582, doi:10.1002/15222683(200208)23:16<2569::AID-ELPS2569>3.0.CO;2-M.

134. Lau, A. Y.; Lee, L. P.; Chan, J. W. An integrated optofluidic platform for Ramanactivated cell sorting. Lab. Chip 2008, 8, 1116-1120, doi:10.1039/b803598a.

135. Casabella, S.; Scully, P.; Goddard, N.; Gardner, P. Automated analysis of single cells using Laser Tweezers Raman Spectroscopy. The Analyst 2016, 141, 689-696, doi:10.1039/C5AN01851J.

136. Dochow, S.; Krafft, C.; Neugebauer, U.; Bocklitz, T.; Henkel, T.; Mayer, G.; Albert, J.; Popp, J. Tumour cell identification by means of Raman spectroscopy in combination with optical traps and microfluidic environments. Lab. Chip 2011, 11, 1484-1490, doi:10.1039/c0lc00612b.

137. Hanson, C.; Israelsen, N. D.; Sieverts, M.; Vargis, E. Fabricating a UV-Vis and Raman spectroscopy immunoassay platform. JoVE J. Vis. Exp. 2016, e54795, doi:10.3791/54795.

138. Israelsen, N. D.; Wooley, D.; Hanson, C.; Vargis, E. Rational design of Ramanlabeled nanoparticles for a dual-modality, light scattering immunoassay on a polystyrene substrate. J. Biol. Eng. 2016, 10, 2, doi:10.1186/s13036-015-0023-y. 
139. Förster-Zuegel, F.; Grotepaß, T.; Schlaak, H. F. Characterization of the dielectric breakdown field strength of PDMS thin films: thickness dependence and electrode shape. In; 2015; Vol. 9430, pp. 94300D-94300D-9.

140. Gerratt, A. P.; Bergbreiter, S. Dielectric breakdown of PDMS thin films. $J$. Micromechanics Microengineering 2013, 23, 067001, doi:10.1088/09601317/23/6/067001.

141. Sano, M. B.; Salmanzadeh, A.; Davalos, R. V. Multilayer contactless dielectrophoresis: theoretical considerations. Electrophoresis 2012, 33, 19381946, doi:10.1002/elps.201100677.

142. Shafiee, H.; Caldwell, J. L.; Davalos, R. V. A microfluidic system for biological particle enrichment using contactless dielectrophoresis. Jala 2010, 15, 224-232, doi:10.1016/j.jala.2010.02.003.

143. Čemažar, J.; Douglas, T. A.; Schmelz, E. M.; Davalos, R. V. Enhanced contactless dielectrophoresis enrichment and isolation platform via cell-scale microstructures. Biomicrofluidics 2016, 10, 014109, doi:10.1063/1.4939947.

144. Elvington, E. S.; Salmanzadeh, A.; Stremler, M. A.; Davalos, R. V. Label-free isolation and enrichment of cells through contactless dielectrophoresis. Jove-J. Vis. Exp. 2013, e50634, doi:10.3791/50634.

145. Salmanzadeh, A.; Elvington, E. S.; Roberts, P. C.; Schmelz, E. M.; Davalos, R. V. Sphingolipid metabolites modulate dielectric characteristics of cells in a mouse ovarian cancer progression model. Integr. Biol. 2013, 5, 843-852, doi:10.1039/c3ib00008g.

146. Sano, M. B.; Gallo-Villanueva, R. C.; Lapizco-Encinas, B. H.; Davalos, R. V. Simultaneous electrokinetic flow and dielectrophoretic trapping using perpendicular static and dynamic electric fields. Microfluid. Nanofluidics 2013, 15, 599-609, doi:10.1007/s10404-013-1175-z.

147. Sano, M. B.; Caldwell, J. L.; Davalos, R. V. Modeling and development of a low frequency contactless dielectrophoresis (cDEP) platform to sort cancer cells from dilute whole blood samples. Biosens. Bioelectron. 2011, 30, 13-20, doi:10.1016/j.bios.2011.07.048.

148. Henslee, E. A.; Sano, M. B.; Rojas, A. D.; Schmelz, E. M.; Davalos, R. V. Selective concentration of human cancer cells using contactless dielectrophoresis. Electrophoresis 2011, 32, 2523-2529, doi:10.1002/elps.201100081.

149. Shafiee, H.; Sano, M. B.; Henslee, E. A.; Caldwell, J. L.; Davalos, R. V. Selective isolation of live/dead cells using contactless dielectrophoresis (cDEP). Lab. Chip 2010, 10, 438-445, doi:10.1039/b920590j.

150. Sano, M. B.; Henslee, E. A.; Schmelz, E. M.; Davalos, R. V. Contactless dielectrophoretic spectroscopy: examination of the dielectric properties of cells found in blood. Electrophoresis 2011, 32, 3164-3171, doi:10.1002/elps.201100351.

151. Salmanzadeh, A.; Kittur, H.; Sano, M. B.; Roberts, P. C.; Schmelz, E. M.; Davalos, R. V. Dielectrophoretic differentiation of mouse ovarian surface epithelial cells, macrophages, and fibroblasts using contactless dielectrophoresis. Biomicrofluidics 2012, 6, 024104, doi:10.1063/1.3699973. 
152. Farmehini, V.; Rohani, A.; Su, Y.-H.; Swami, N. S. A wide-bandwidth power amplifier for frequency-selective insulator-based dielectrophoresis. Lab. Chip 2014, 14, 4183-4187, doi:10.1039/c4lc00801d.

153. Klein, N.; Gafni, H. The maximum dielectric strength of thin silicon oxide films. IEEE Trans. Electron Devices 1966, ED-13, 281-289, doi:10.1109/TED.1966.15681.

154. Jones, P. V.; Hayes, M. A. Development of the resolution theory for gradient insulator-based dielectrophoresis. Electrophoresis 2015, 36, 1098-1106, doi:10.1002/elps.201400504.

155. Song, Y.; Sonnenberg, A.; Heaney, Y.; Heller, M. J. Device for dielectrophoretic separation and collection of nanoparticles and DNA under high conductance conditions: Nanoanalysis. ELECTROPHORESIS 2015, 36, 1107-1114, doi:10.1002/elps.201400507.

156. Jones, P. V.; Salmon, G. L.; Ros, A. Continuous separation of DNA molecules by size using insulator-based dielectrophoresis. Anal. Chem. 2017, 89, 1531-1539, doi:10.1021/acs.analchem.6b03369.

157. Sonnenberg, A.; Marciniak, J. Y.; Skowronski, E. A.; Manouchehri, S.; Rassenti, L.; Ghia, E. M.; Widhopf, G. F.; Kipps, T. J.; Heller, M. J. Dielectrophoretic isolation and detection of cancer-related circulating cell-free DNA biomarkers from blood and plasma. Electrophoresis 2014, 35, 1828-1836, doi:10.1002/elps.201400016.

158. Srivastava, S. K.; Daggolu, P. R.; Burgess, S. C.; Minerick, A. R. Dielectrophoretic characterization of erythrocytes: Positive ABO blood types. ELECTROPHORESIS 2008, 29, 5033-5046, doi:10.1002/elps.200800166.

159. Faraghat, S. A.; Hoettges, K. F.; Steinbach, M. K.; van der Veen, D. R.; Brackenbury, W. J.; Henslee, E. A.; Labeed, F. H.; Hughes, M. P. Highthroughput, low-loss, low-cost, and label-free cell separation using electrophysiology-activated cell enrichment. Proc. Natl. Acad. Sci. U. S. A. 2017, 114, 4591-4596, doi:10.1073/pnas.1700773114.

160. Qiang, Y.; Liu, J.; Mian, M.; Du, E. Experimental electromechanics of red blood cells using dielectrophoresis-based microfluidics. In Mechanics of Biological Systems and Materials, Volume 6; Conference Proceedings of the Society for Experimental Mechanics Series; Springer, Cham, 2017; pp. 129-134 ISBN 978-3319-41350-1.

161. Chwang, A. T.; Wu, T. Y. Hydromechanics of low-Reynolds-number flow. Part 4. Translation of spheroids. J. Fluid Mech. 1976, 75, 677-689, doi:10.1017/S0022112076000451.

162. Hawkins, B. G.; Huang, C.; Arasanipalai, S.; Kirby, B. J. Automated dielectrophoretic characterization of Mycobacterium smegmatis. Anal. Chem. 2011, 83, 3507-3515, doi:10.1021/ac2002017. 
APPENDICES 


\section{APPENDIX A}

\section{COPY RIGHTS AND PERMISSIONS}

MDPI Terms of Use (Chapter 5)

$\S 1$ These Terms of Use govern the use of the MDPI websites or any other MDPI online services you access. This includes any updates or releases thereof. By using our online services, you are legally bound by and hereby consent to our Terms of Use and Privacy Policy. These Terms of Use form a contract between MDPI AG, registered at St. Alban-Anlage 66, 4052 Basel, Switzerland (“MDPI”) and you as the user (“User”). These Terms of Use shall be governed by and construed in accordance with Swiss Law, applicable at the place of jurisdiction of MDPI in Basel, Switzerland.

$\S 2$ Unless otherwise stated, the website and affiliated online services are the property of MDPI and the copyright of the website belongs to MDPI or its licensors. You may not copy, hack or modify the website or online services, or falsely claim that some other site is associated with MDPI. MDPI is a registered brand protected by the Swiss Federal Institute of Intellectual Property.

$\S 3$ Unless otherwise stated, articles published on the MDPI websites are labeled as “Open Access” and licensed by the respective authors in accordance with the Creative Commons Attribution (CC-BY) license. Within the limitations mentioned in $\S 4$ of these Terms of Use, the “Open Access” license allows for unlimited distribution and reuse as long as appropriate credit is given to the original source and any changes made compared to the original are indicated. 
$\S 4$ Some articles published on this website (especially articles labeled as "Review" or similar) may make use of copyrighted material for which the author(s) have obtained a reprint permission from the copyright holder. Usually such reprint permissions do not allow author(s) and/or MDPI to further license the copyrighted material. The licensing described in $\S 3$ of these terms and conditions are therefore not applicable to such kind of material enclosed within articles. It is the User's responsibility to identify reusability of material provided on this website, for which he may take direct contact with the authors of the article.

$\S 5$ You may register or otherwise create a user account, user name or password (your “Registration”) that allows you to access or receive certain content and/or to participate or utilize certain features of our online service, including features in which you interact with us or other users. You represent and warrant that the information provided in your Registration is accurate to the best of your knowledge. You are responsible for the use of any password you create as part of your Registration and for maintaining its confidentiality, and you agree that MDPI may use this password to identify you. We reserve the right to deny, terminate or restrict your access to any content or feature reached via such Registration process for any reason, at our sole discretion. MDPI reserves the right to block or to terminate the User's access to the website at any time and without prior notice.

$\S 6$ The MDPI website and online services may provide links to other websites or external resources. As part of these Terms of Use, you acknowledge that MDPI is in not responsible for the availability of such external sites or resources, and that 
MDPI is not liable for any content, services, advertising, or materials available from such external sites or resources.

$\S 7$ The website may contain advertising. MDPI does not endorse any responsibility of any kind for the content of the advertisement or sponsorship or the advertised product or service, which is the responsibility of the advertiser or sponsor, unless the advertised product or service is offered by MDPI.

$\S 8$ There is no warranty for the website and its content, to the extent permitted by applicable law. MDPI, the copyright holders and/or other parties provide the website and its content “as is” without representations or warranties of any kind, either expressed or implied, including, but not limited to, the implied warranties of merchantability, satisfactory quality and fitness for a particular purpose relating to this website, its content or any to which it is linked. No representations or warranties are given as to the accuracy or completeness of the information provided on this website, or any website to which it is linked.

$\S 9$ In no event, unless required by applicable law shall MDPI, its employees, agents, suppliers, contractors or any other party, be liable to the User for any damages of any nature, including any general, special, incidental or consequential damages, loss, cost, claim or any expense of any kind arising out of the use, inability to access, or in connection with the use of the website, its content and information, even if the User has been advised of the possibility of such damages. 
$\S 10$ MDPI reserves the right to change these Terms of Use at any time by posting changes to this page of the website without prior notice. Please check these Terms of Use periodically for any modifications. Your continued use of any Service following the posting of any changes will mean that you have accepted and agreed to the changes.

$\S 11$ Basel, Switzerland shall be the place of jurisdiction for all legal disputes arising of these Terms of Use, even if the Customer has her/his domicile outside of Switzerland.

$\S 12$ Swiss law applicable at the place of jurisdiction of MDPI shall apply exclusively.

$\S 13$ If any provisions of the Terms of Use should be found invalid, this shall not affect the validity of the remaining provisions. In any such case, the contracting parties shall negotiate on the invalid clause to substitute by a valid arrangement as close as possible to the original provision. 
For Chapter 3

$5 / 31 / 2017$

Gmall - RE: permission for reuse

M Gmail

Cindy Hanson <chanson8491@gmail.com>

\section{RE: permission for reuse}

1 message

PermissionsUK <PermissionsUK@sagepub.com>

To: Cindy Hanson <chanson8491@gma'.com>

Mon, Apr 17, 2017 at 12:15 PM

Dear Cindy Hanson,

Thank you for your email. I am pleased to report we can grant your request without a fee as part of your dissertation.

Please accept this email as permission for your request as detailed below. Permission is granted for the life of the edition on a non-exclusive basis, in the English language, throughout the world in all formats provided full citation is made to the original SAGE publication.

The permission is subject to approval from any co-authors on the original project. Please note approval excludes any graphs, photos, excerpts, etc. which required permission from a separate copyright holder at the time of publication. If your material includes anything which was not your original work, please contact the rights holder for permission to reuse those items.

Best Wishes,

Craig Myles

on behalf of SAGE Ltd. Permissions Team

SAGE Publishing

1 Oliver's Yard, 55 City Road

London, EC1Y 1SP

UK

www.sagepublishing.com

SAGE Rublications Ltd, Registered in Endind No.1017514

Los Angeles | London | New Delh

Singapore | Wastington DC

The natural home for authors, edlion \& societies

Thank you for considering the environment before printing this emat. 
From: Cindy Hanson [mailto:chanson8491@gmail.com]

Sent: Monday, April 17, 2017 11:06 AM

To: PermissionsUK <PermissionsUK@sagepub.com>

Subject: permission for reuse

Hello,

I'm the author of the article "Effect of Principal Component Analysis Centering and Scaling on Classification of Mycobacteria from Raman Spectra" published in Applied Spectroscopy last year. As the article is not an open access article, I believe I need to obtain copyright permission to incorporate it in my dissertation. Is there a form I need to fill out to do this?

Cheers!

$-$

Cindy Hanson

Graduate Research Assistant

Utah State University

4105 Old Main Hill ENGR 402

Logan, UT 84322 


\section{APPENDIX B}

\section{PERMISSION FROM AUTHORS TO REPRINT PUBLISHED MATERIALS}

May 31, 2017

To whom it may concern:

My name is Michael Sieverts. Cynthia Hanson and I wrote the manuscript titled: "Effect of principal component analysis centering and scaling on classification of mycobacteria from Raman spectra." This paper was published in Applied Spectroscopy in 2016. Cindy was first author and major contributor to this work. I give her permission to reprint the manuscript in its entirety in her PhD dissertation.

Sincerely,

Michael Sieverts 


\section{APPENDIX C}

\section{R CODE}

\#R-code for statistical classification methods using mycobacteria spectra

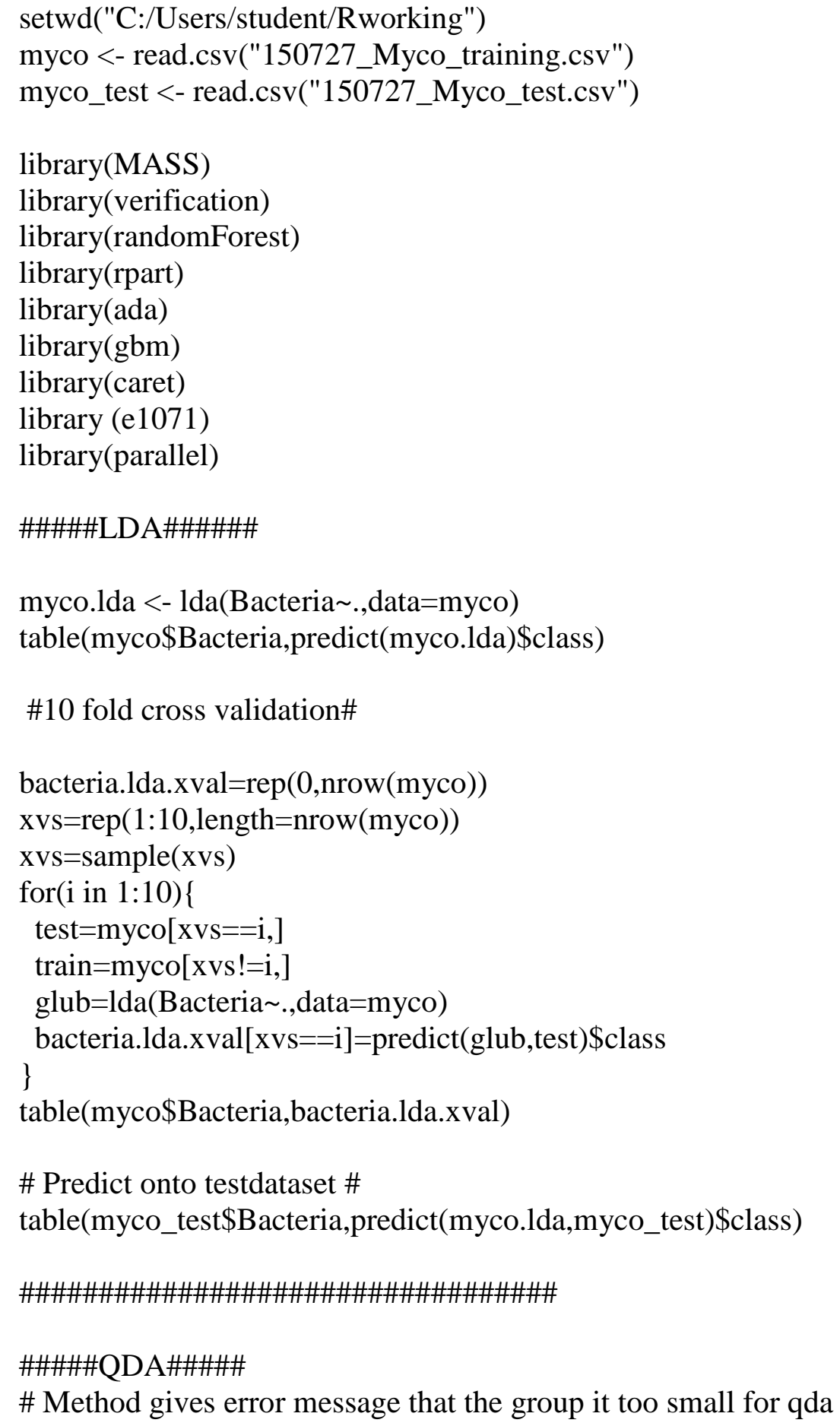




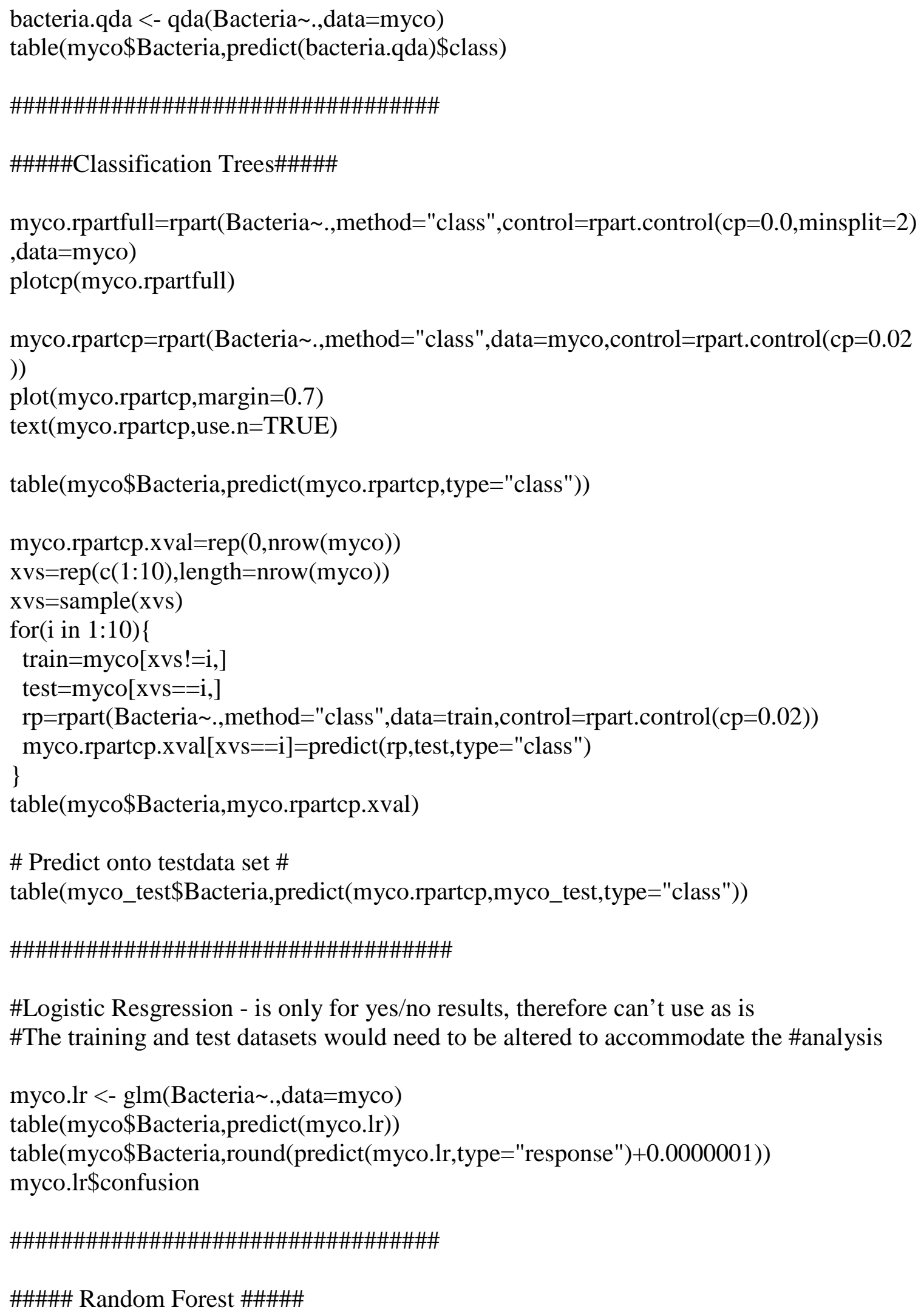


myco.rf <- randomForest(as.factor(Bacteria) .,data=myco,keep.forest $=$ T)

myco.rf.predict <- predict(myco.rf)

table(myco\$Bacteria,myco.rf.predict)

myco.rf\$confusion

\# Predict onto testdata set \#

table(myco_test\$Bacteria,predict(myco.rf,myco_test,type="class"))

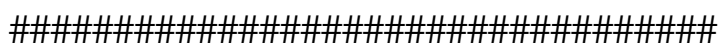

\#\#\#\#\#)upport Vector Machines\#\#\#\#\#

myco.svm=svm(Bacteria .,method="class",data=myco)

table(myco\$Bacteria,predict(myco.svm,type="class"))

myco.svm.xval=rep $(0, \operatorname{nrow}($ myco $))$

xvs=rep(c(1:10),length=nrow(myco) $)$

xvs=sample(xvs)

for(i in $1: 10)\{$

train=myco[xvs!=i, ]

test $=$ myco[xvs $==\mathrm{i}$, $]$

svm=svm(Bacteria .,method="class",data=train)

myco.svm.xval[xvs==i]=predict(svm,test,type="class")

\}

table(myco\$Bacteria,myco.svm.xval)

\# Predict onto testdata set \#

table(myco_test\$Bacteria,predict(myco.svm,myco_test,type="class"))

\#\#\#\#\#radient Boosted Trees\#\#\#\#\#

myco.gbm=gbm(Bacteria .,distribution="gaussian",n.trees=5000,data=myco)

table(myco\$Bacteria,round(predict(myco.gbm,

type="response",n.trees $=5000)+0.0000001)$ )

myco.gbm.xvalpr=rep(0,nrow(myco))

xvs=rep(1:10,length=nrow(myco))

xvs=sample(xvs)

for(i in 1:10)\{

train=myco[xvs!=i, ]

test $=$ myco[xvs $==\mathrm{i}$, ]

glub=gbm(Bacteria .,distribution="gaussian",n.trees=5000,data=train)

myco.gbm.xvalpr[xvs==i]=predict(glub,newdata=test,type="response",n.trees=5000)

\} 
table(myco\$Bacteria,round(myco.gbm.xvalpr+0.0000001))

\# Prediction onto testdata set \#

table(myco_test\$Bacteria,round(predict(myco.gbm,newdata=myco_test,type="response", n.trees $=5000)+0.0000001)$ )

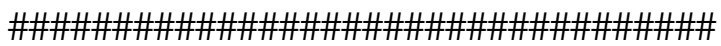

\#\#\#\#\#Tuning GBM\#\#\#\#\#

fitControl $=$ trainControl $($ method $=$ "cv", number $=10)$

\# Code to use more than one processor \#

$\mathrm{cl}<$ - makeCluster(4)

gbmGrid=expand.grid(.interaction.depth $=c(5,10,15)$, .n.trees $=c(100,200,300)$,

.shrinkage $=\mathrm{c}(0.02,0.1,0.2))$

gbmFit=train(as.factor(Bacteria) ., method="gbm", tuneGrid=gbmGrid,

trControl=fitControl, data=myco)

gbmFit

\# Results: 300 n.trees, 15 interaction depth, 0.02 shrinkage

\#keep performing iterations of different ranges for interaction depth, tree size, and \#shrinkage until optimal values are found for each. The following code is an example

gbmGrid=expand.grid(.interaction.depth $=c(15)$, .n.trees $=c(300,350,400)$, shrinkage $=$ $\mathrm{c}(0.01,0.02))$

gbmFit=train(as.factor(Bacteria) ., method="gbm", tuneGrid=gbmGrid,

trControl=fitControl, data=myco)

gbmFit

\# Results: 300 n.trees, 15 interaction depth, 0.02 shrinkage

gbmGrid=expand.grid(.interaction.depth $=c(15,20,25)$, .n.trees $=c(300)$, .shrinkage $=$ $\mathrm{c}(0.02))$

gbmFit=train(as.factor(Bacteria) ., method="gbm", tuneGrid=gbmGrid, trControl=fitControl, data=myco)

gbmFit

\# Results: 300 n.trees, 25 interaction depth, 0.02 shrinkage

gbmGrid=expand.grid(.interaction.depth $=c(25,30)$, .n.trees $=c(300)$, shrinkage $=$ $\mathrm{c}(0.02))$

gbmFit=train(as.factor(Bacteria) ., method="gbm", tuneGrid=gbmGrid, trControl=fitControl, data=myco)

gbmFit 
\# Results: 300 n.trees, 30 interaction depth, 0.02 shrinkage

gbmGrid=expand.grid $($ interaction $\cdot$ depth $=c(30,35,40)$, .n.trees $=c(300)$, shrinkage $=$ $c(0.02))$

gbmFit=train(as.factor(Bacteria) ., method="gbm", tuneGrid=gbmGrid, trControl=fitControl, data=myco)

gbmFit

\# Results: 300 n.trees, 30 interaction depth, 0.02 shrinkage

myco.gbm2=gbm(Bacteria .,distribution="gaussian",interaction.depth=30,n.trees=300, shrinkage $=0.02$, data $=$ myco)

table(myco\$Bacteria,round(predict(myco.gbm2,type="response",n.trees=300)+0.000000

1))

\#crossvalidation\#

myco.gbmopt.xvalpr=rep(0,nrow(myco))

xvs=rep $(1: 10$,length=nrow(myco) $)$

xvs=sample(xvs)

for(i in 1:10)\{

train $=$ myco[xvs!=i, $]$

test $=$ myco[xvs $==\mathrm{i}$, $]$

glub=gbm(Bacteria .,distribution="gaussian",interaction.depth=30,n.trees=300, shrinkage $=0.02$, data $=$ train)

myco.gbmopt.xvalpr[xvs==i]=predict(glub,newdata=test,type="response",n.trees=300) \}

table(myco\$Bacteria,round(myco.gbmopt.xvalpr+0.0000001))

\# Predict onto testdata set \#

table(myco_test\$Bacteria,round(predict(myco.gbm2,newdata=myco_test,type="response

",n.trees=300)+0.0000001))

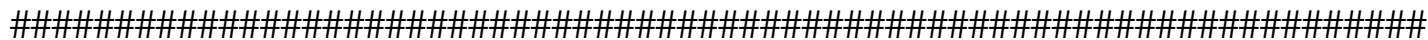

\#R-code for to gather data as described in article for Applied Spectroscopy

\#the code includes a loop to generate confusion matrices for all PC possible for a

\#particular dataset

\#change the set directory to the file where the datasets are stored setwd("C:/Users/Cindy/Documents/R/Datasets")

\#change the name of the file as you move through each training and test dataset\#

myco10 <- read.csv("Raman_Train_Data_1.csv")

myco10_test $<$-read.csv("Raman_Test_Data_1.csv")

library(MASS) 


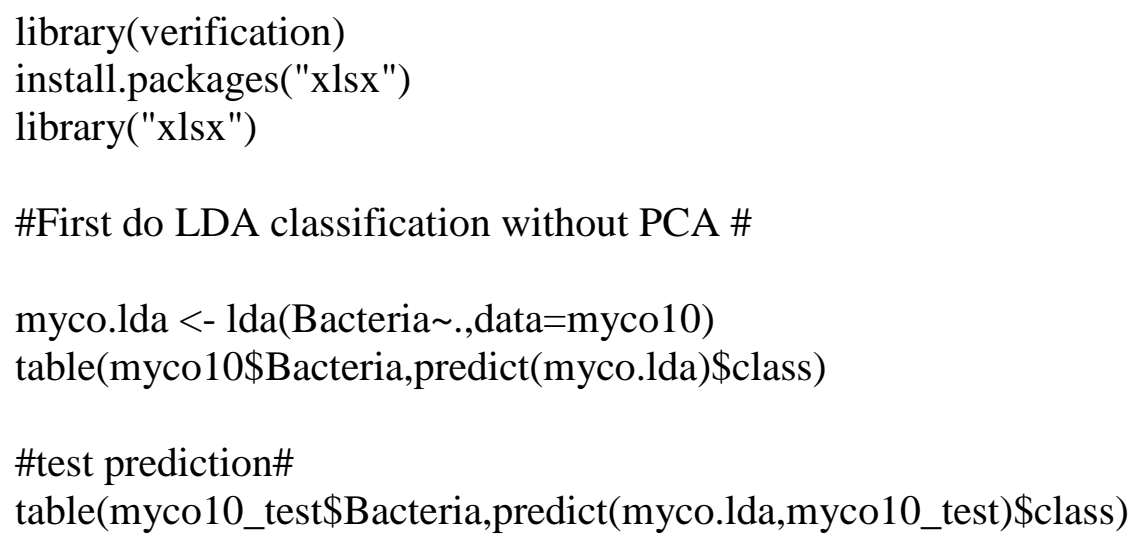


myco.bacteria.prep <- myco10_test[,1]

myco.bacteria.prep.mat <- as.matrix(myco.bacteria.prep)

is.matrix(myco.bacteria.prep.mat)

\#The test dataset needs to experience the same rotation as the training dataset predict(myco.pca,myco.test.prep)

myco.test.pca <- predict(myco.pca,myco.test.prep)

myco10.test.pcs <- cbind(myco.bacteria.prep.mat,myco.test.pca)

myco10.test.pca.df <- as.data.frame(myco10.test.pcs)

\# write out to tables \#

write.table(myco10.pca.dataframe,file="Raman_Data_6_Training_NC\&S.csv",row.name s=FALSE,na="',col.names=TRUE,sep=",")

write.table(myco10.test.pca.df,file="Raman_Data_6_Test_NC\&S.csv",row.names=FALS E,na="',col.names=TRUE,sep=",")

\#Read in the files and give it an appropriate name

myco10_PCA_Train_all <-read.csv("Raman_Data_6_Training_NC\&S.csv")

myco10_PCA_Test_all <-read.csv("Raman_Data_6_Test_NC\&S.csv")

\# The following code is a loop that will return the resulting confusion matrix for \#predicting the test dataset according to each PC. Make sure the results_train.csv and \#results_test.csv is either empty or has zeros for A1-C3.

\# V1 is "Bacteria"\#

for(i in 2:ncol(myco10_PCA_Train_all)) \{

myco10_PCA_Train <- (myco10_PCA_Train_all[,1:i])

myco10_PCA_Test <-(myco10_PCA_Test_all[,1:i])

myco10.lda $<-$ lda(V1 .,data=myco10_PCA_Train)

$\mathrm{x}<$-table(myco10_PCA_Train\$V1,predict(myco10.lda)\$class)

y<-table(myco10_PCA_Test\$V1,predict(myco10.1da,myco10_PCA_Test)\$class)

mat.x <- as.matrix $(\mathrm{x})$

write.table(mat.x,file="outfile_x.csv",sep=",", col.names = FALSE, row.names =

FALSE)

olddata_x <-read.csv("results_train.csv",header=FALSE,sep=",")

mat.olddata_x $<$ - as.matrix(olddata_x)

newdata_x <-rbind(mat.olddata_x,mat.x)

write.table(newdata_x,file="results_train.csv",sep=",", col.names = FALSE, row.names $=$ FALSE)

mat.y <- as.matrix $(y)$

write.table(mat.y,file="outfile_y.csv",sep=",", col.names = FALSE, row.names = FALSE)

olddata_y<-read.csv("results_test.csv", header=FALSE, sep=",")

mat.olddata_y <- as.matrix(olddata_y) 
newdata_y <-rbind(mat.olddata_y,mat.y)

write.table(newdata_y,file="results_test.csv",sep=",", col.names = FALSE, row.names = FALSE)

\}

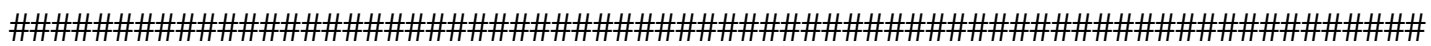

\#R-code for creating test and training datasets in a format that can be used in $\mathrm{R}$

\# prior to building, spectra for a particular bacteria is given a name and is saved in the \# working directory and is randomly assigned to be in the training or test dataset.

setwd("C:/Users/Cindy/Documents/R/Datasets")

library(MASS)

library(verification)

library(randomForest)

library(rpart)

library(ada)

library(gbm)

library(caret)

library (e1071)

library("xlsx")

library(gtools)

\#Raman

\#Building the Training Dataset

readin_R1 <- read.csv("RJ6.csv",header=TRUE,sep=",")

readin_R2 <- read.csv("RJ8.csv",header=TRUE,sep=",")

readin_R3 <- read.csv("RJ9.csv",header=TRUE,sep=",")

readin_R4 <- read.csv("RK5.csv",header=TRUE,sep=",")

readin_R5 <- read.csv("RK7.csv",header=TRUE,sep=",")

readin_R6 <- read.csv("RK8.csv",header=TRUE,sep=",")

readin_R7 <- read.csv("RM5.csv",header=TRUE,sep=",")

readin_R8 <- read.csv("RM7.csv",header=TRUE,sep=",")

readin_R9 <-read.csv("RM8.csv",header=TRUE,sep=",")

mat_R1 <- as.matrix(readin_R1)

mat_R2 <- as.matrix(readin_R2)

mat_R3 <- as.matrix(readin_R3)

mat_R4 <- as.matrix(readin_R4)

mat_R5 <- as.matrix(readin_R5)

mat_R6 $<$ - as.matrix(readin_R6)

mat_R7 <- as.matrix(readin_R7)

mat_R8 $<$ - as.matrix(readin_R8)

mat_R9 $<$ - as.matrix(readin_R9) 


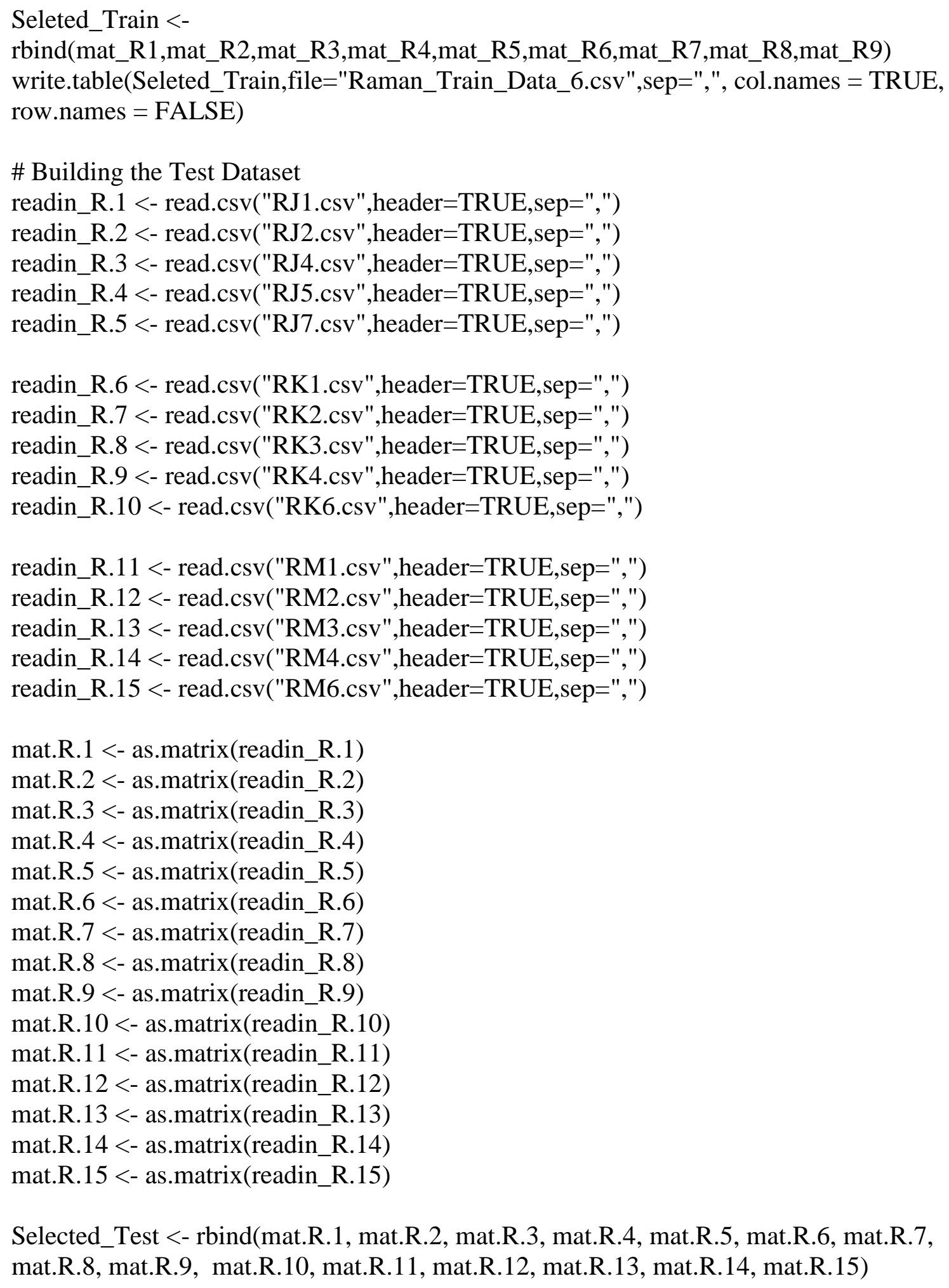

Selected_Test <- rbind(mat.R.1, mat.R.2, mat.R.3, mat.R.4, mat.R.5, mat.R.6, mat.R.7, mat.R.8, mat.R.9, mat.R.10, mat.R.11, mat.R.12, mat.R.13, mat.R.14, mat.R.15) 
write.table(Selected_Test,file="Raman_Test_Data_6.csv",sep=",", col.names = TRUE, row.names $=$ FALSE)

\# adjust code as needed to accommodate the various test and training data sets.

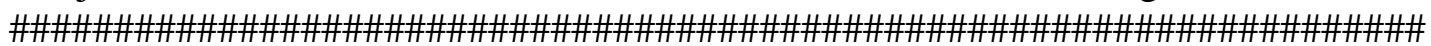

\#R-code for creating LDA plots

\#change the set directory to the file where the datasets are stored setwd("C:/Users/Cindy/Documents/R/Datasets")

library(MASS)

\#read in the file

LB_phase <-read.csv("LB_Compiled.csv")

\#LDA\#

LB.lda <- lda(Name .,data=LB_phase)

table(LB_phase\$Name,predict(LB.lda)\$class)

plot(LB.lda, panel $=$ function $(\mathrm{x}, \mathrm{y}, \ldots)$ points $(\mathrm{x}, \mathrm{y}, \ldots)$, col = as.integer(LB_phase\$Name), pch $=19)$

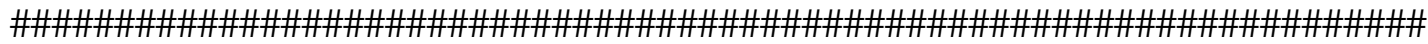




\section{APPENDIX D}

\section{COMSOL OPERATING PARAMETERS}

This section includes the information to set up a model of the cDEP device to acquire estimated $\nabla \mathrm{E}$ values. These values are important to determine if the design is capable of trapping particles as explained in Chapter 6.

Upon opening COMSOL, select the following choices:

- $\quad$ Model Wizard

- $2 \mathrm{D}$

- $\mathrm{AC} / \mathrm{DC}$

O Add Electric Currents

o Add Frequency Domain for Study

- Done

\section{Global Parameters}

Under Global Definitions, enter the information in the following table:

Table D.1. Table of parameters under Global Definitions.

\begin{tabular}{|c|c|c|}
\hline Name & Expression & Description \\
\hline SCW & $500 \mathrm{e}-6[\mathrm{~m}]$ & Sample channel width \\
\hline Ncol & 6 & Number of colums in pillar array \\
\hline Nrow & 5 & Number of rows in pillar array \\
\hline pil_diam & $(\mathrm{SCW}-(\mathrm{Nrow}+1) * \mathrm{Sb}) /$ Nrow & Diameter of pillars in array \\
\hline $\mathrm{Sb}$ & $33 e-6[m]$ & Space between pillars in array \\
\hline PW & $25 \mathrm{e}-3[\mathrm{~m}]$ & Plate width \\
\hline PL & $50 \mathrm{e}-3[\mathrm{~m}]$ & Plate length \\
\hline BW & $20 \mathrm{e}-6[\mathrm{~m}]$ & Barrier width \\
\hline RW & SCW & LE width \\
\hline RL & $15 \mathrm{e}-3[\mathrm{~m}]$ & LE length \\
\hline AL & $2.4 \mathrm{e}-3[\mathrm{~m}]$ & Length from edge to LE \\
\hline QW & $5 e-3[\mathrm{~m}]$ & LE leg height \\
\hline $\mathrm{QL}$ & SCW & LE leg width \\
\hline
\end{tabular}




\section{Build Geometry}

1. Rectangle (r1)
a. Width: PL
b. Height: PW
c. Position > Base: Center at $(0,0)$

2. Rectangle (r2)
a. Width: PL
b. Height: SCW
c. Position $>$ Base: Center at $(0,0)$

3. Rectangle (r3)
a. Width: RL
b. Height: RW
c. Position > Base: Corner
i. $\mathrm{x}:-0.5 * \mathrm{PL}+\mathrm{AL}$
ii. $\mathrm{y}: 0.5 * \mathrm{SCW}+\mathrm{BW}$

4. Rectangle (r4)
a. Width: SCW
b. Height: QW
c. Position > Base: Corner
i. $\mathrm{x}:-0.5 * \mathrm{PL}+\mathrm{AL}$
ii. $\mathrm{y}: 0.5 * \mathrm{SCW}+\mathrm{BW}$

5. Rectangle (r5)
a. Width: QL
b. Height: QW
c. Position > Base: Corner
i. $\mathrm{x}:-0.5 * \mathrm{PL}+\mathrm{AL}+\mathrm{RL}-\mathrm{SCW}$
ii. $\mathrm{y}: 0.5 * \mathrm{SCW}+\mathrm{BW}$

6. Union (uni2)

a. Select objects r3, r4, and r5

b. "Keep input objects” and "Keep interior boundaries” unchecked

7. Mirror (mir1)
a. Select object uni2
b. Check box "Keep input objects"
c. Point on Line of Reflections $(0,0)$
d. Normal Vector to Line of Reflection $(1,0)$

8. Mirror (mir2)
a. Select objects uni2 and mir1
b. Check box "Keep input objects"
c. Point on Line of Reflections $(0,0)$
d. Normal Vector to Line of Reflection $(0,1)$ 
9. Circle (c1)

a. Radius: $0.5 *$ pil_diameter

b. Sector angle: 360

c. Position > Base: Center

i. $\mathrm{x}:-(0.5 * \mathrm{Ncol}-0.5) *($ pil_diam $+\mathrm{Sb})$

ii. y: $-(0.5 *$ Nrow -0.5$) *($ pil_diam $+\mathrm{Sb})$

10. Array (arr2)

a. Select object c1

b. Array type: Rectangular

i. $\mathrm{x}$ size: Ncol

ii. y size: Nrow

c. Displacement

i. x: pil_diam+Sb

ii. y: pil_diam+Sb

\section{Build All}

To build the array of ovals, values for Nrow and Sb change to 4 and 33e-6[m] respectively. In addition, the parameter "scale" is added with a value of 0.6. After step 9 the procedure changes as follows:

10. Scale (sca1)

a. Input object c1

b. Uncheck "Keep input objects" box

c. Scale Factor

i. Scaling: anisotropic

ii. $\mathrm{x}$ : scale

iii. $\mathrm{y}: 1$

d. Center of scaling $(0,0)$

11. Array

a. Select object sca1

b. Array type: Rectangular

i. $\mathrm{x}$ size: Ncol

ii. y size: Nrow

c. Displacement

i. $x$ : scale*pil_diam+Sb

ii. y: pil_diam+Sb

12. Build All 


\section{$\underline{\text { Add Materials }}$}

Under Component $1>$ Materials, add quartz from the materials library. Also add the following information for blank material:

- Sample channel

o Electrical conductivity: $0.005 \mathrm{~S} / \mathrm{m}$

o Relative permittivity: 80

- Liquid electrodes

o Electrical conductivity: $1.5 \mathrm{~S} / \mathrm{m}$

o Relative permittivity: 80

Under each material, select the areas that correspond with each material.

\section{$\underline{\text { Assign Source and Sink Electrodes }}$}

Under Component $1>$ Electrical Currents

- $\quad$ Select the Physics tab

- Click the down arrow under Boundaries

- Select Terminal

o Select the boundaries for liquid electrodes which correspond to the source electrodes

o Under Terminal drop down, select Voltage as the terminal type

o Enter the desired voltage for the source electrode

- Repeat for the process to add a sink electrode with the voltage set to $0+$

$\underline{\text { Add a Study }}$

- $\quad$ Select the Study tab

- Click Add Study and the Add study pane will appear on the right hand side

- Select Frequency Domain

- Click Add Study

- Under the Frequency Domain Settings tab find study settings and enter the desired frequency

- Compute

\section{$\underline{\text { Add a 2D plot group to determine } \nabla\left(\mathrm{E}^{2}\right)}$}

- $\quad$ Select the Results tab

- Select 2D Plot group

- Select Surface

- In the Settings pane, find Expression 
- Click the Replace Expression drop down arrow

- $\quad$ Select Model $>$ Component $1>$ Electric Currents $>$ Electric field $>$ ec.Ex Electric field, $\mathrm{x}$ component

- Under Expression

o Type d(ec.Ex^2,x)

0 Unit should change to $\mathrm{kg} \wedge 2 * \mathrm{~m} /\left(\mathrm{s}^{\wedge}{ }^{*} * \mathrm{~A} \wedge 2\right)$

- Click Plot in the Settings pane 


\section{APPENDIX E}

\section{LASTER ALIGNMENT ONTO SPECTROMETER SLIT}

Collection of Raman spectra requires proper alignment of the laser spot through the objective and imaged onto the spectrometer slit. The process of aligning the laser is documented here. Please refer to Figure E.1 to identify important components in laser alignment.

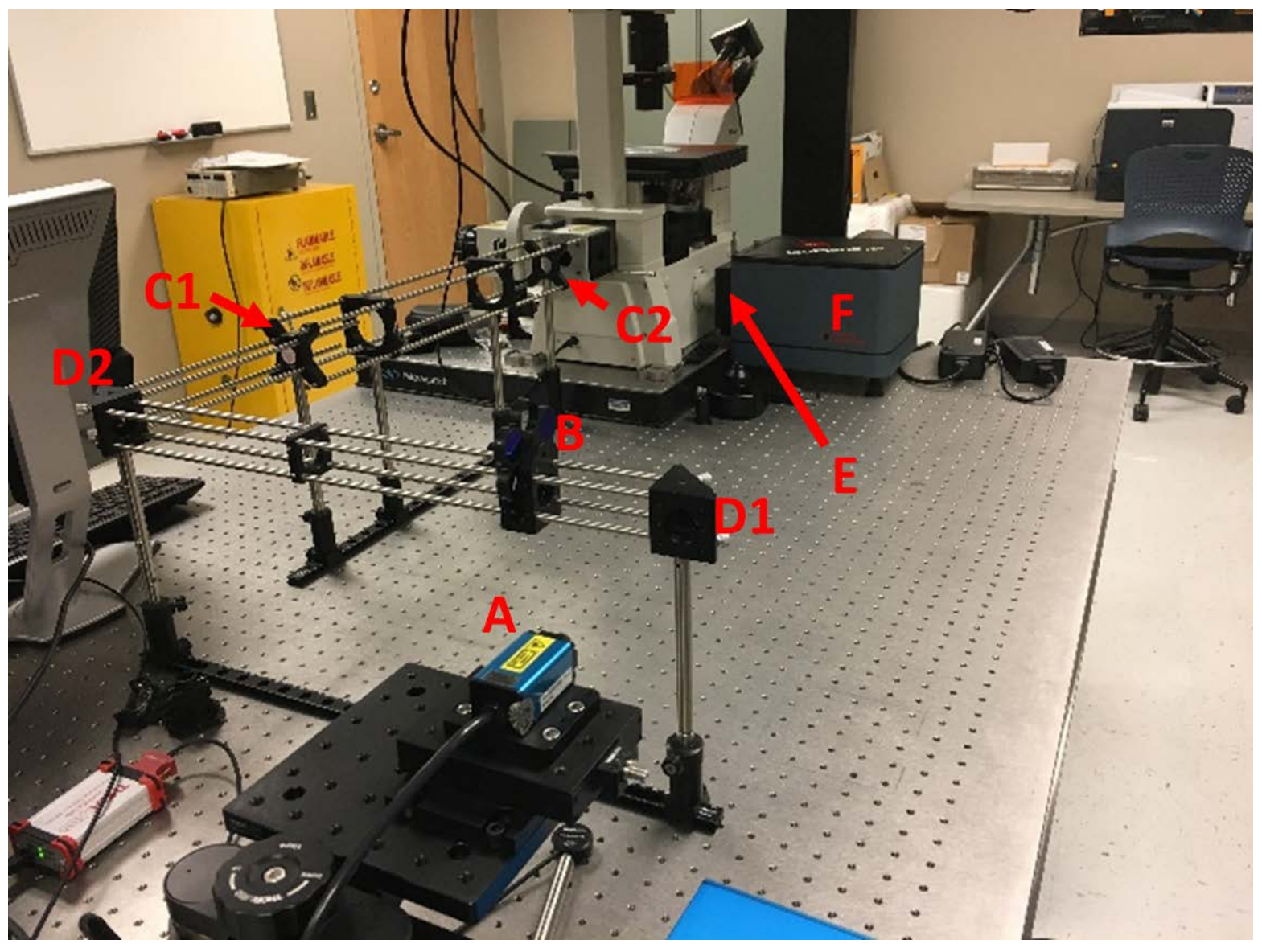

Figure E.1. Image of laser delivery to in-house built Raman microscope.

Important components include laser head (A), filter wheel with neutral density filters (B), $60 \mathrm{~mm}$ to $30 \mathrm{~mm}$ adapter plates with threaded hole to accommodate alignment target (C1 and C2), mirrors in kinematic mounts (D1 and D2), entrance to spectrometer slit $(\mathrm{E})$, and the spectrometer $(\mathrm{F})$. 
Before the laser is aligned to the spectrometer, a rough alignment must first be accomplished. This is done using a D1, D2, and an fluorescent illumination target (Thorlabs VRC4SM1) using a Z-fold alignment as shown in Figure E.1. Ensure that the laser and the center of the all optics are at the same height. The laser is then centered on the target at C1 by adjusting D1. The target is then removed from C1 and placed into C2. The laser is centered at C2 by adjusting D2. The process is repeated until the laser is centered at C1 and C2 without the need of further adjustment to D1 and D2. This rough alignment will allow for the laser spot to be visible on the spectrometer opening

Fine tune alignment first requires determining the center of the spectrometer by acquiring an image of light coming through the entrance slit with the grating acting like a mirror (set center wavelength to 0 using Lightfield software). The image will look like Figure E.2. After the center of the spectrometer is determined, remove the slit and add a neutral density filter into the filter cube at the location of the emission filter as indicated by Figure E.4. Also insert a neutral density filter in the laser path using the fly wheel as shown in Figure E.1. Collect an image of the laser spot, which will look similar to Figure E.3. Before acquiring and image using the spectrometer, make sure to do the following:

- Neutral density filter is in place

- $\quad$ Slit wide open

- $\quad$ Center wavelength set at 0

- Focus objective on second surface

Adjust the laser spot to the center using kinematic mount D2. Then add a fluorescent target to C2 and center laser spot using kinematic mount D1. Repeat this centering 
process until the laser is aligned at C2 and at the spectrometer. Note that the centering process should be done on a substrate that is the same thickness that will be used when acquiring Raman spectra for the sample.

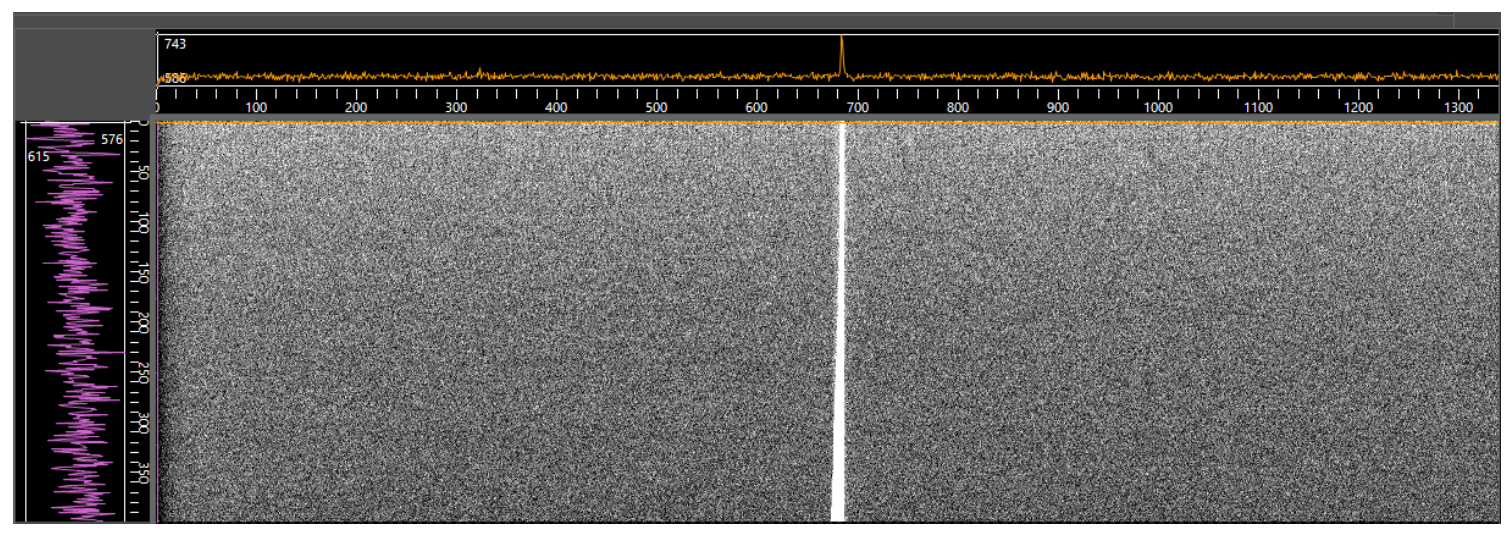

Figure E.2. Image of light entering through the spectrometer slit to determine vertical and horizontal midpoint for laser alignment.

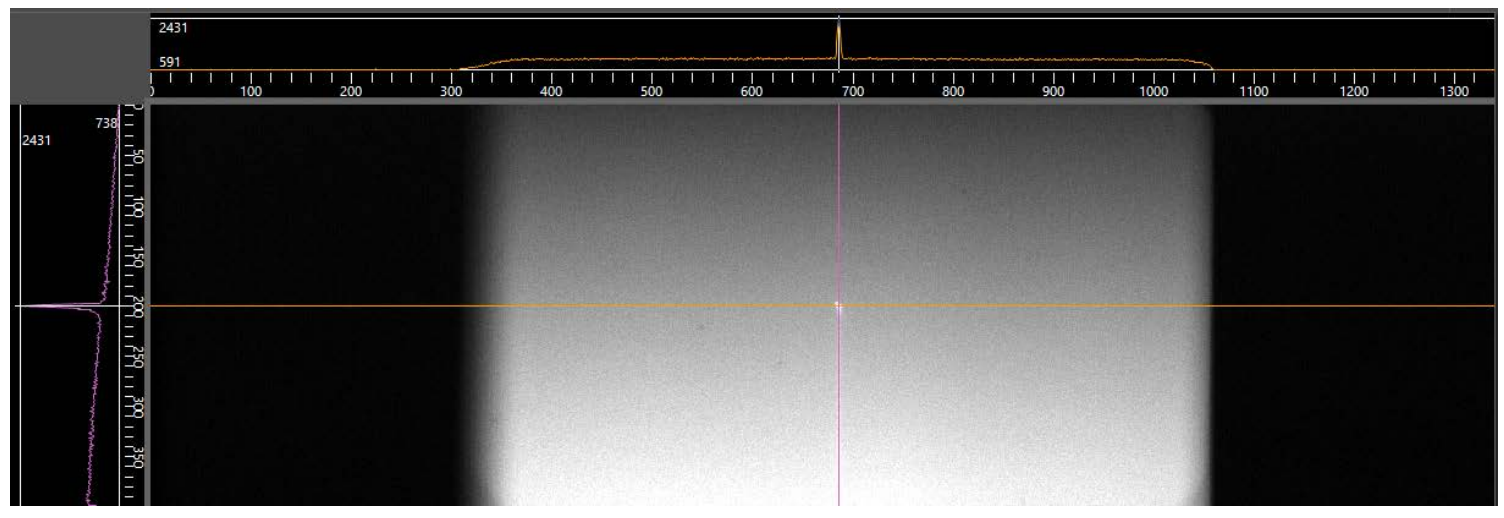

Figure E.3. Image of laser spot being aligned to the center of the spectrometer slit as determined by Figure E.2. 


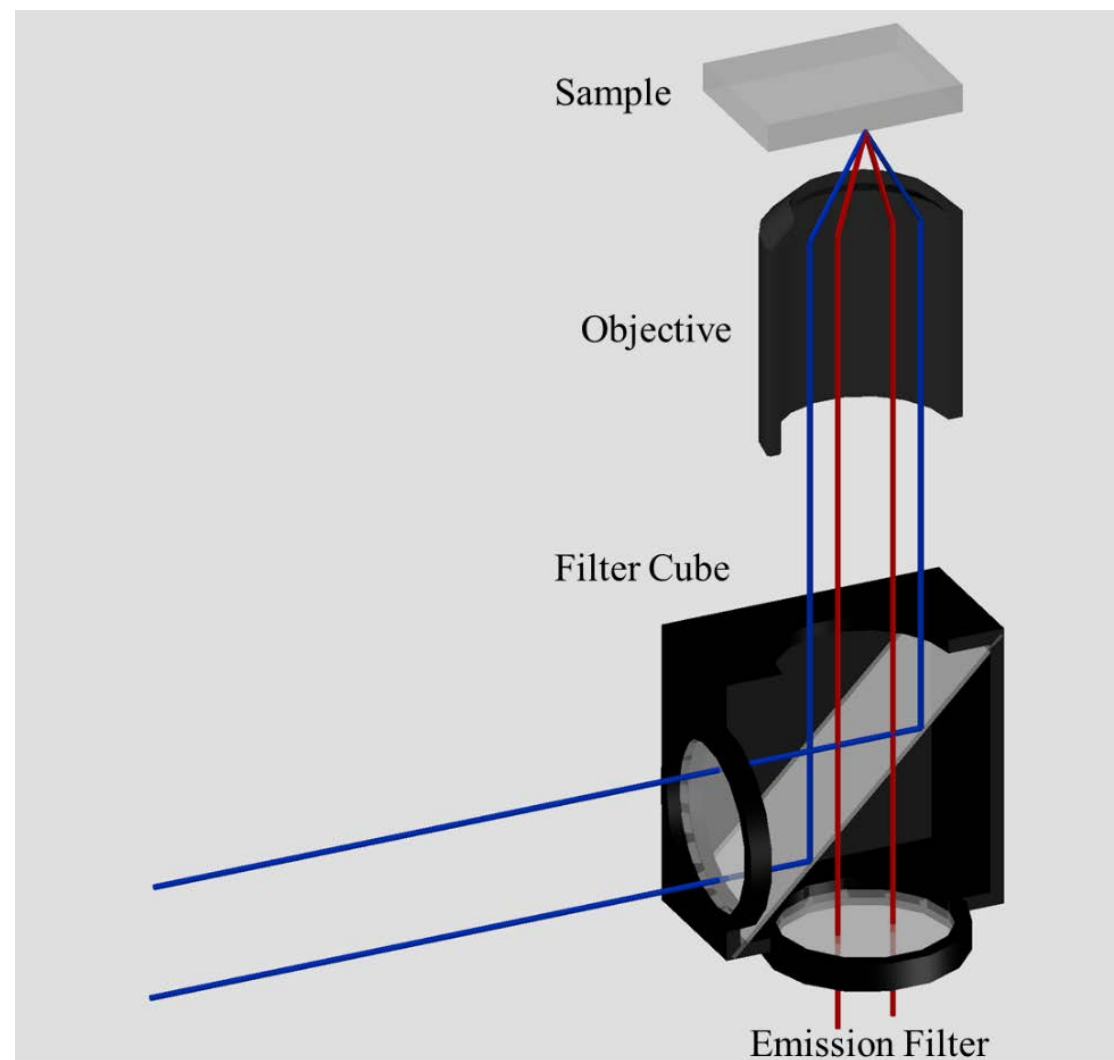

Figure E.4. Illustration of filter cube used in the in-house built Raman microscope. Blue and red lines represent the excitation laser and the scattered light, respectively. 


\section{CURRICULUM VITA}

\section{Cynthia Hanson}

chanson8491@gmail.com

\section{EDUCATION}

Ph.D. Biological Engineering

May 2018

Utah State University - Logan, UT

M.S. Nuclear Engineering

May 2013

University of Idaho - Idaho Falls, ID

Thesis: "Laser-Induced Breakdown Spectroscopy (LIBS) -

Temperature Effects on Spectra of Molten Salt Systems”

B.S. Chemical Engineering

May 2008

University of Idaho - Moscow, ID

Senior Project: AIChE Chem Car Competition

A.A. Liberal Arts

May 2003

College of Southern Idaho - Twin Falls, ID

\section{CERTIFICATIONS}

Nuclear Criticality Safety

University of Idaho - Idaho Falls, ID

May 2013

Laboratory Assistant

College of Southern Idaho - Twin Falls, ID

May 2003

\section{PROFESSIONAL TRAINING}

Radiation Training

Radiation Worker

Hazardous Materials Shipper

Hazardous Waste Shipper

RCRA Hazardous Waste Regulations

Shipping Radioactive Materials

Environmental Sampling and Data

Analysis

Health Physics

Radiation Worker
Center for Advanced Energy

Studies

Idaho National Laboratory

HMTC Training \& Consulting

HMTC Training \& Consulting

McCoy and Associates, Inc.

FedEx

Envirostat, Inc.

Naval Reactors Facility

Naval Reactors Facility
Aug 2011

Jun 2011

Feb 2010

Feb 2010

Apr 2009

Apr 2009

Jan 2009

Dec 2008

Dec 2008

\section{PROFESSIONAL MEMBERSHIPS}

- Society of Women Engineers: 2005 - present

- Society for Applied Spectroscopy: 2011 - present 
- SPIE: 2013 - present

- American Electrophoresis Society: 2015 - present

\section{EXPERIENCE}

\section{Graduate Research Assistant}

Utah State University, Logan, UT

Aug 2013 -

- Set-up biophotonics lab

Present

- Train undergraduate and graduate students in laser alignment, maintenance, safety, and operation

- Perform research using Raman and SERS for the detection and identification of bacterial samples

- Prepare and present research at technical conferences

- Write and publish research in appropriate scientific journals

\section{Engineering Intern}

Idaho National Laboratory, Idaho Falls, ID

- Coordinated communication among nine stakeholders to

May 2011 -

Sept 2012

and establish documentation to safely perform laser

May 2013 spectroscopy

Aug 2013

- Designed and built a chamber to analyze molten salts using LIBS

- Collected data to demonstrate the influence of atmospheric pressure on LIBS spectra

- Prepared and presented research at the 2012 International Pyroprocessing Research Conference

\section{Graduate Research and Teaching Assistant}

University of Idaho, Idaho Falls, ID

- Analyzed laser-induced break down spectroscopy (LIBS) spectra of molten salt to determine direction of future studies

Aug 2010 May 2011

and Oct 2012 -

Dec 2012

- Wrote and defended a thesis on the effect of salt temperature on LIBS spectra of molten salt systems

- Designed and performed experiments to track the adsorption qualities of select concrete appetites for potential use in an interim storage facility

- Graded homework and responded to students' questions regarding course content

\section{Waste and Shipping Associate Engineer}

Naval Reactors Facility, Idaho Falls, ID

- Characterized and managed radioactive, PCB, RCRA 
- Wrote and peer-reviewed technical work documents for handling radioactive waste

- Trained employees in waste disposal process

- Liaison between NRF and off-site analytical laboratories

- Reviewed and approved radioactive recycle metal forms

- Proposed improvements for waste review processes

Laboratory Technician

Anatek Labs, Moscow, ID

- Performed laboratory extractions for pesticides, fats, oils, and greases

- Performed routine maintenance on laboratory equipment

\section{Engineering and Laboratory Assistant}

TIC Gums, Belcamp, MD

- Investigated bleaching procedure and provided recommendations for process improvement

- Performed quality assurance tests of carbohydrate gums

Research Intern

Iowa State University, Ames, IA

- Researched and presented findings concerning an alternative wastewater treatment method

\section{Laboratory Technician}

Idaho Food Quality Assurance Laboratory, Twin Falls, ID

- Performed pesticide extractions

- Trained new employees on pesticide extractions

- Prepared calibration curves, primary standards and mixes

- Wrote standard operating procedures

- Performed routine maintenance on specialized lab equipment
May 2007 -

May 2008

Summer

2006

Summer 2005

May 2001 -

May 2003

\section{HONORS AND AWARDS}

USU Dissertation Fellowship - May 2017

USU Student Research Symposia Graduate Poster Presentation Award - April 2017

Utah State University Graduate Research and Creative Opportunities Award - Dec 2016

USTAR Student Originated Project Award - Oct 2016

USU College of Engineering PhD Travel Grant - August 2016

Outstanding Graduate Researcher for USU Biological Engineering Department - Jan

2016

Best student poster for Devices section at NanoUtah - October 2015

SPIE Optics and Photonics Scholarship - May 2015

Utah State University Graduate Enhancement Award - April 2015

RGS Graduate Student Travel Award - 2014, 2015, 2016 


\section{PUBLICATIONS}

- $\quad$ C. Hanson, E. Vargis, "Alternative cDEP design to facilitate cell isolation for identification by Raman spectroscopy,” Sensors, 2017, 17, 327, doi: 10.3390/s17020327.

- C. Hanson, E. Vargis, "Effect of PCA centering and scaling on classification of mycobacteria from Raman spectra,” Applied Spectroscopy, First published: 25 Nov 2016, doi: 10.1177/0003702816678867.

- C. Hanson*, N.D. Israelsen*, M. Sieverts, E. Vargis, "Fabricating a UV-Vis and Raman spectroscopy immunoassay platform,” Journal of Visualized Experiments, 2016, 117, e54795, doi:10.3791/54795.

- N.D. Israelsen, D. Wooley, C. Hanson, E. Vargis, "Rational design of Ramanlabeled nanoparticles for a dual-modality, light scattering immunoassay on a polystyrene substrate," Journal of Biological Engineering 2016, 10:2.

- C. Hanson, E. Vargis, “Open-system Raman microscopy,” Laser Focus World 2015, 51, 71-73.

- N.D. Israelsen*, C. Hanson*, E. Vargis, "Nanoparticle properties and synthesis effects on surface-enhanced Raman scattering enhancement factor: an introduction,” The Scientific World Journal, vol. 2015, Article ID 124582, 12 pages, 2015. doi:10.1155/2015/124582.

- $\quad$ C. Hanson, S. Phongikaroon, J.R. Scott, "Temperature effect on laser-induced breakdown spectroscopy spectra of molten and solid salts," Spectrochimica Acta Part B - Atomic Spectroscopy 2014, 97, 79-85.

- J.J. Hatch, T.R. McJunkin, C. Hanson, and J.R. Scott, "Automated interpretation of LIBS spectra using a fuzzy logic inference engine,” Applied Optics 2012, 51, B155-B164.

* = Equal contribution

\section{PRESENTATIONS}

- C. Hanson, E. Vargis, "Proposed DEP-Raman device for simultaneous trapping and identification of bacteria," USU Student Research Symposia, Logan, UT, April 13, 2017. [poster]

- C. Hanson, E. Vargis, "Proposed DEP-Raman device for simultaneous trapping and identification of bacteria," Institute of Biological Engineering Annual Conference, Salt Lake City, UT, March 30, 2017. [poster]

- $\quad$ C. Hanson, E. Vargis, "A proposed cDEP device design for improved device reusability and range of applied voltage," Scientific Exchange, Minneapolis, MN, September 22, 2016. [poster]

- C. Hanson, E. Vargis, "Proposed dielectrophoretic design to sort and identify bacteria,” USU Graduate Research Symposia, Logan, UT, April 14, 2016. [oral]

- $\quad$ C. Hanson, E. Vargis, "The use of microfluidics and dielectrophoresis for separation, concentration, and identification of bacteria," Photonics West, San Francisco, CA, February 14, 2016. [oral] 
- C. Hanson, E. Vargis, “A proposed method to isolate, concentrate, and identify bacteria by dielectrophoresis and Raman spectroscopy,” AIChE Annual Meeting, Salt Lake City, UT, November 9, 2015. [poster]

- $\quad$. Hanson, E. Vargis, “A proposed method to isolate, concentrate, and identify bacteria by dielectrophoresis and Raman spectroscopy," nanoUtah, Salt Lake City, UT, October 13, 2015. [poster]

- C. Hanson, E. Vargis, "Comparison of machine learning methods to identify bacteria using Raman spectroscopy,” Scientific Exchange, Providence, RI, September 30, 2015. [poster]

- C. Hanson, "Dr. Vargis Lab: biomedical photonics and tissue engineering," Annual Hansen Life Sciences Retreat, Logan, UT, September 19, 2015. [oral]

- C. Hanson, E. Vargis, "Effect of PCA on spectral classification of mycobacteria," Annual Hansen Life Sciences Retreat, Logan, UT, September 19, 2015. [poster]

- $\quad$ C. Hanson, E. Vargis, "Use of surface-enhanced Raman spectroscopy to identify mycobacteria,” USU Graduate Research Symposia, Logan, UT, April 9, 2015. [oral]

- C. Hanson, E. Vargis, "Use of SERS magnetic nanoparticles to concentrate and identify mycobacteria,” nanoUtah, Salt Lake City, UT, October 13, 2014. [poster]

- $\quad$ C. Hanson, E. Vargis, “Application of SERS magnetic nanoparticles to concentrate, detect, and identify mycobacteria," Scientific Exchange, Reno, NV, September 29, 2014. [oral]

- $\quad$ C. Hanson, E. Vargis, “A simple method for bacterial concentration, detection and identification using SERS magnetic nanoparticles,” USU Graduate Research Symposia, Logan, UT, April 10, 2014. [oral]

- $\quad$ C. Hanson, J.J. Hatch, S. Phongikaroon, J.R. Scott, “Laser-induced breakdown spectroscopy (LIBS): temperature effects on spectra of molten salt systems," University of Idaho Engineering Design Expo, Moscow, ID, April 26, 2013. [poster]

- $\quad$ C. Hanson, S. Phongikaroon, J.J. Hatch, and J.R. Scott, "Laser-induced breakdown spectroscopy (LIBS): temperature effects on spectra of molten salt systems," University of Idaho Master's Thesis Defense, Idaho Falls, ID, April 22, 2013. [oral]

- $\quad$ C. Hanson, S. Phongikaroon, J.J. Hatch, and J.R. Scott, "Effects of temperature variation on LIBS spectra of molten salt," International Pyroprocessing Research Conference, Fontana, WI, August 26-29, 2012. [oral]

- $\quad$ C. Hanson, J. Beck, M. Piekarski, "Glucose oxidase enzyme battery powered car with iodine clock timing reaction,” University of Idaho Engineering Design Expo, Moscow, ID April 25, 2008. [poster]

- C. Hanson, J. Beck, T. Kooda, “Bio-chemical model car propulsion,” University of Idaho Engineering Design Expo, Moscow, ID, April 25, 2008. [oral]

- $\quad$ C. Hanson, S. Khanal, "The effects of ultrasound on aerobic digestion of waste activated sludge,” Iowa State University, Program for Women in Science and Engineering, Ames, IA, July 22, 2005. [poster] 